
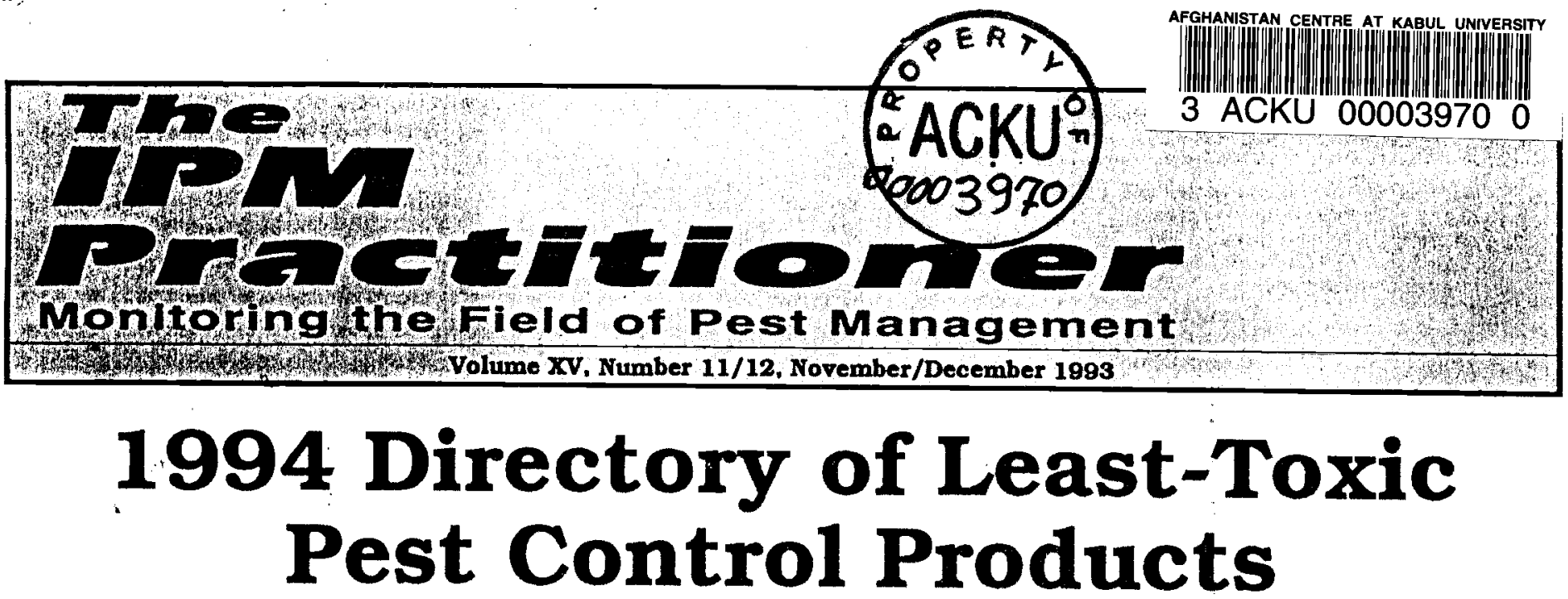

$\mathrm{T}$ The 1994 Directory is a gateway to more than a thousand useful pest control items (for quick access see Box A). Many of these products, services, or beneficial organisms have come to our attention in connection with articles published in BIRC's IPM Practitioner or the Common Sense Pest Control Quarterly, through IPM programs designed by our staff, or via recommendations from colleagues.

This Dircctory is unique because it is compiled by IPM technical experts, includes specific product descriptions, and is organized in concert with the IPM decisionmaking process (sce The IPM Practitioner 13(11/12):1-9). All information is current, and is constantly updated on BIRC's computcrized database system. It is divided into four management sections: Insects, Plant Disease, Vertebrates, and.Weeds. Within eacls section, information is listed in alphabetical order by specific pest grouping. The IPM decision-making steps used to organize individual pest listings include Identification \& Munitoring plus a consideration of Physical, Horticultural, Biological, and Least-Toxic Chenical control methods.

For each product, specific descriptions are included when applicable, followed by the company name and the product name when available (in parentheses). Addresses and phone numbers of manufacturers and suppliers are listed alphabetically at the end of the Directory. An Index to the Directory follows the List of Addresses.

\section{Feedback and Disclaimer}

Two caveats apply to this information. First, to the best of our knowledge, these products, services, and beneficials are effective and are produccd by reputable companies. However, they are used at your

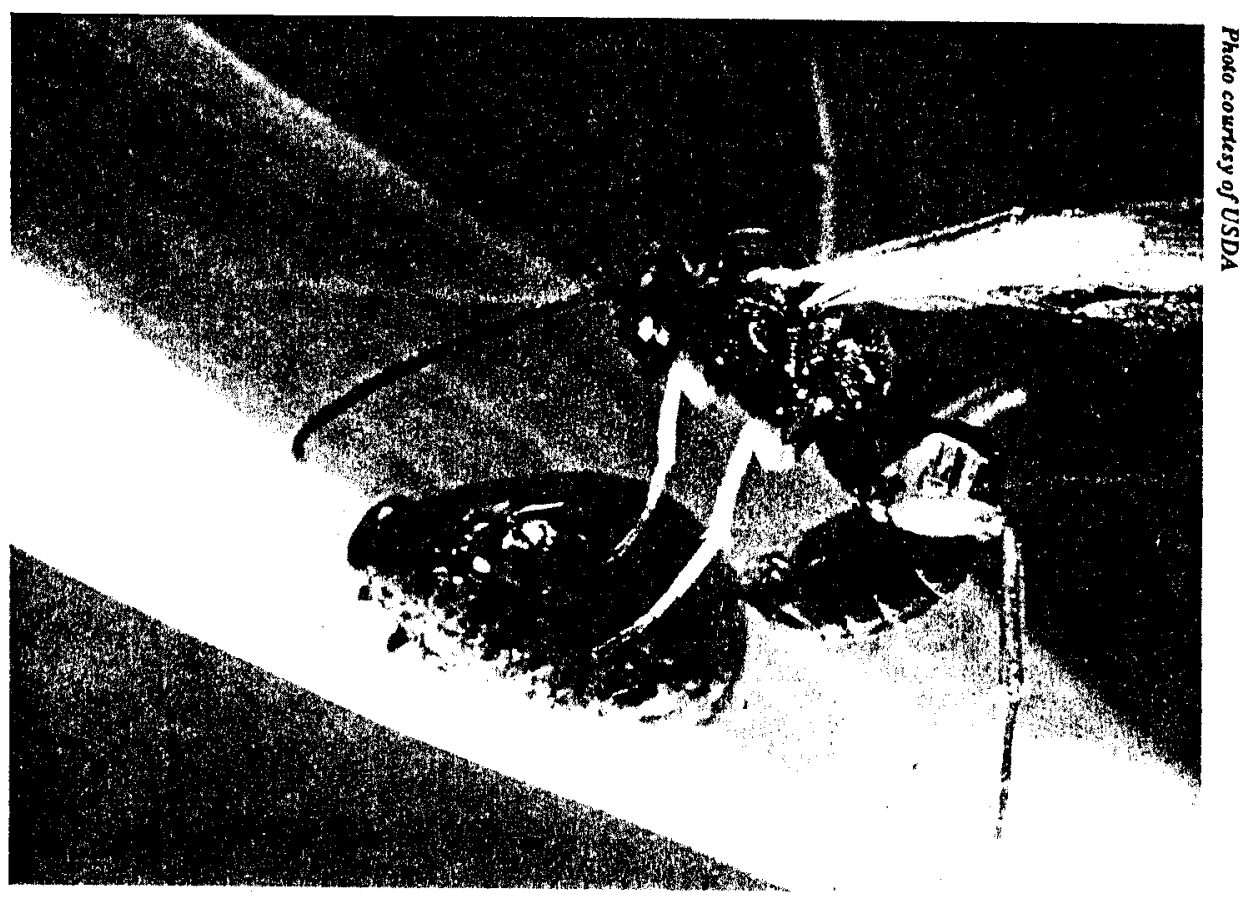

The parasitold Lemophagus sp. lays eggs in a larval cereal leaf beetle, Oulema melanopus.

own risk, and BIRC makes no guarantees or warr anties regarding any listing. To help us maintain quality control, we encourage you to contact us about your experiences, both positive and negative, with any of thesc companies or resources

Sccondly, all information was current at press time. However, pest control is a rapidly changing field: products come and go, and companies change hands and locations fairly frequently. Therefore, if you fail to reach any of the companies listed here, contact us at PO Box 7414, Berkeley, CA 94707; 510/524-2567.

\section{Beneficial Insects}

Both domestic and international producers of beneficials found in the Directory are listed by alphabetical order in the List of Addresses at the end of the Directory. Names and addresses of intemational Koppert beneficial insect distributors are listed separately in a Box at the beginning of this address list.

To obtain speedy delivery and receive advice from experienced sources, we recommend that you order directly from producers. Most of the producers listed rear at least one benefical, but may distribute organisms produced by other companies.

Some producers may refer you to a distributor for small quantities. Whether the company is a producer or a distributor can be determined when a company representative is contacted. Since space limitations preclude listing all retail distributors of 
beneficial insects, only a select few are included.

International Orders of Beneficials.

The IPM Practitioner is published ten times per year by the Blo-integral Resource Conter (BIRC), a non-profit corporation underlaking research and education in integrated pest management.

Editors

Sheila Daer Helga Olkowski William Olkowsk

Assoclate Editors Williarn Quarles Laurie Swiadon

Contrlbuting Edltors

Kerry Beane Larry Burkam Joel Grossman Shawn King Jamie Liebman Anghe Zhang

\section{Membership and}

Subscription Manager

Databese Manager

Advertising Menager

Joe Posner

Renata Brillinger

Kerry Beane

Lisa Dumas

Shea Godwin

Kathy Spalding

For media kits or other advertising information, contact Renala Brillinger at 510/524-2567.

\section{Advisory Board}

George Bird, Michigan State Univ.; Sterling Bunnell, M.D., Berkeley, CA ; Paul Catts, Washinglon Slate Univ.; Momei Chen, Acad. of Forestry, Beiling, China; David Davis, No. Carolina State Univ., Emer.; Walter Eboling. UCLA, Emer.; Steve Frantz, NY State Dept. Health; Joseph Hancock, Univ. Calit, Berkeley; Mantred Mackaver, Centre for Pest Management, Simon Fraser Univ., Canada; Robent Melcalf, Univ. Illinois; Goorge Poinar, Univ. Calit, Berkeley; Ron Prokopy, Univ. Massa. chusetts; Ramesh Chandra Saxena, In'll Rice Research Instifute, Manila, Philippines; Ruth Troestschler, Zoecon Corporation; J.C. van Lenteren, Agricultural University Wageningen. The Netherlands; Aen Wang, Biological Control Inst., CAAS., Beijing. China.

\section{Menuscrlpls}

The IPMP welcomes accounts of IPM for any pest situation. Write for details on format for manuscripts.

\section{Cliations}

Unless otherwise noled, all text is written by Sheila Daar, Helga Olkowski, \& William Olkowsk. Permission to reprint is granted if source is fully acknowledged and notified.

\section{Subscrlptlons/Memberships}

A subscription to the IPMP is one of the benelits of membership in BIRC. We also answer pest management questions lor our members and heip them search for information. Memberships are $\$ 50 / y r$ (institutions/ librarles/businesses); $\$ 25 / y r$ (individuals); $\$ 18$ yr (registered student). Canadian subscribers add $\$ 10$ poslage. All other foreign subscribers add $\$ 20$ airmail postage. A Dual membership, which includes a combined subscription to both the IPMP and the Common Sense Pest Control Quarterly, cosis $\$ 75 / y r$ (institutions); $\$ 45 / y r$ (individuals). Government purchase orders accepled. Donations to BIRC are taxdeductibTe. F.El\# 94-2554036.

\section{Change of Address}

When writing to request a change of address please send a copy of a recent address label.
If you are looking for a specific item, see the Index on pages 31 and 32 . To find a remedy for your pest problem, look in one of the four major sections according to whether the pest is an insect (arthropod), plant disease pathogen, vertebrate, or a weed. Except for the weed section, pests are listed by alphabetical order in each of the major categories. When you have found the pest listing, there are separate sections for Identification and Monitoring, and for Physical, Horticultural, Biological, or Least-Toxic Chemical controls. For instance, to find a parasitoid to control aphids, look in the Insect Management section under Aphids, then under Biological Controls, then under Parasitoids. To find a trap for a raccoon, look in the Vertebrate section under Raccoons, then under Physical Controls until you find a listing of traps. Brand name items are enclosed in parentheses, and are attached to a series of suppliers. The first supplier on the list is the manufacturer.

If the product you are seeking is not in the Index, you must know how the product is used, and for which pests, in order to immediately find the listing. If the item you are secking is hard to pests (e.g., number per sample leaf or percent of crop infested), and life stage of pest (adult, larva, etc.).

Instructions for Beneficials. Most producers send instructions for releasing beneficial insects. However, these organisms are most effective when used within a full IPM program. Send for BIRC's Publications Catalogue (available for $\$ 1$ postpaid) for a listing of 'how-to' articles on incorporating biological control organisms within IPM programs for specific pests.

Acknowledgements: We wish to thank all the producers who furnished updated information for this Directory. A special vote of thanks goes to Dr. Joop van Lenteren, a member of BIRC's Scientific Advisory Board, for providing information on intemational producers of beneficial organisms. Our thanks also to the Apple Computer Corporation for donation of computer equipment.

\section{Box A. Quick Access}

càtegorize, each of the four main divisions has a Miscellaneous section. If you are looking for a specific item such as insecticidal soap, diatomaceous earth, or silica gel, and do not know which pest category you should look at, browse major pest sections such as Ants and Aphids first, then browse Miscellaneous.

\section{Beneficials}

To find beneficial insects or arthropods, look first in the Index. If there is no listing in the Index, find the appropriate major section, then the alphabetical listing of the pest you are trying to control. When you have found the pest listing, then look under Biological Controls. Parasitoids available to fight your pest are listed first, followed by effective predators. Beneficials are listed in alphabetical order by scientific name.

A list of suppliers follows every item in the Directory. To find the addresses and phone numbers for the suppliers listed, look up each supplier by alphabetical order in the List of Addresses at the end of the Directory. 


\section{INSECT MANAGEMENT}

\section{ANTS}

\section{Identification \& Monitoring}

Trained Dogs (for Carpenter Ants):

Industrial Narcotic - TADD

Vacuums:

Bon-Aire (Bon-Aire SuperVac)

\section{Physical Controls}

Caulk:

Whitmire, Brody (Pest Proof Caulk)

Heat Treatment (carpenter ants):

Isothermics (Call for local practitioners)

- Mission Pest Control

Sticky Barrier:

Intagra (Bug Gum) - Tanglefoot, Bozeman, Gardenville, Gardens Alive!, Great Lakes, Harmony, Necessary, Phero Tech (Tanglefoot)

Tree Wrap:

Olson (Stiky Tree Guards) • Intagra (Tree Banding Tape) • Seabright,

Great Lakes, Harmony, Phero Tech (Stickem Green)

\section{Biological Controls}

Microbial Insecticides:

Abamectin (for fire ants):

Whitmire, Gardenville (PT-370 Ascend)

Parasitic Mites:

Pyemotes tritici: (for fire ant): ARBICO $\cdot$ Blofac $\cdot$ Praxis

\section{Least-Toxic Chemical Controls}

Borate-Based Insecticides:

Waterbury (Mop-Up) - Whitmire (PT 240 or PT 249 Perma-Dust) • Roxide (Roxo) - Waterbury, Gardener's Supply, Gardenville, Harmony (Drax) • Harmony (Revenge) • Peaceful Valley

Borate rods (for carpenter ants):

CSI, NISUS (Impel Rods)

Borate treatments (for carpenter ants):

NISUS (Bora-Care) • U.S. Borax (TimBor)

Diatomaceous Earth:

Natural Insecto (Insecto) • Organic Plus

- Permaguard, Nitron (Permaguard)

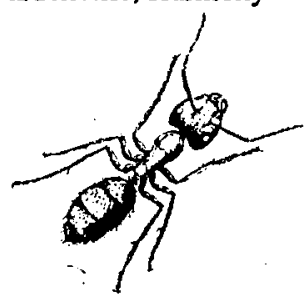

- Earth Tools, Natural Insect (Insectigone) • Necessary • Harmony •

Gardenville

With pyrethrin:

Diacide (Homeguard) - Permaguard, Pristine, Nitron, Gardenville

(Permaguard Household) • Organic Plus

Hydramethylnon:

Clorox Company (Maxforce, Combat)
Insect Growth Regulators:

Fenoxycarb (for fire ants):

Ciba/Geigy, Target, Gardenville, PBI/Gordon (Logic)

Methoprene (for pharoah ants):

Zoecon (Pharorid)

Insecticidal Soap:

With citrus oil:

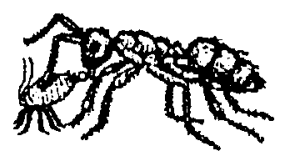

Koppent (Savona) - Ringer, Gardens Alive!, Peaceful Valley,

Gardenville (Safer Crawling Insect Attack) - Ringer, Bozeman, Gardens Alive!, Harmony, IFM, Nitron, Johnny's, Smith \& Hawken, Peaceful

Valley, Charley's, Gardenville (Safer Insecticidal Soap)

Pyrethrin:

Roxide - Whitmire (PT 3-6-10 Aero-Cide, PT-565 Plus) • Hess \& Clark (Capsul-Ban) • Roussel, Insects Limited (Kicker)

Repellent Garlic Spray:

Garlic Research Labs, Agape (Garlic Barrier) - Guardian Spray, New

Earth (ENVIRepel, Guardian Spray)

Silica Aerogel:

100\% Pure:

Cabot (Cab-O-Sil) (Must order 10 pound minimum) - Degussa

(Wessalon S., Aerosil 380) (Must order 25 pound minimum)

With ammonium fluosillicate:

Roussel (Dri-Die; 95\% silica aerogel, $2 \%$ ammonium fluosilicate; $3 \%$ inens) - Target (Dri-Die)

With pyrethrin:

Purity Products - Roussel, Insects Limited, Target, Gardenville (Drione) - Roxide, Harmony (Revenge) - Whitmire (PT 230 or PT 239 Tri-Die) •

Van Waters \& Rogers

\section{APHIDS}

\section{Identification \& Monitoring}

Pheromones \& Traps:

Gardens Alive!, Great Lakes, Olson, Pest Management, Trécé

(Pherocon) - Consep, Harmony, IFM, Pest Management (Checkmate) •

Natural Insect, Peaceful Valley (Bug Charm)

\section{Physical Controls}

Barriers \& Exclusion Devices:

Gardens Alive! - Bozeman

\section{Row Covers:}

American Agrifabrics (Agryl) • Gardener's

Supply • Gardens Alive! (Super-Light) •

Great Lakes (Reemay) • Harmony (Reemay,

Tufbell) - Necessary • Peaceful Valley (Reemay,

Tufbell, Agryl, Poly)

Screening:

ATI (Greenhouse screen) • Hydro-Gardens (Fly Barr) • Tie-Net 


\section{Sticky Adhesive:}

Charley's, Great Lakes, Harmony, Phero Tech, Seabright (Stickem Special) - Arbico, Bozeman, Great Lakes, Gardenville, Harmony, Natural Insect Control, Pest Management, Tanglefoot (Tangle-Trap)

\section{Sticky Trap/Tape:}

Arbico - Hansens - Great Lakes, Olson, Pest Management (Yellow Stiky Traps \& Tapes) - Seabright (Sticky Traps) • Phero Tech (Chroma) • Necessary - Scentry, Great Lakes, Arbico, Bozeman, Pest Management (Versagard)

Traps:

Arbico - Bozeman - Gardens Alive! - Gardenville • Great Lakes • Harmony • IFM • IPM Labs • Natural Insect Control • Pest Management

\section{Biological Controls}

Habitat:

Biotochnik (Earwig-Shelter)

\section{Microbial Insecticides:}

Verticillium lecanii fungus for greenhouse use:

Koppent (Mycotal, Vertalec)

Parasitoids:

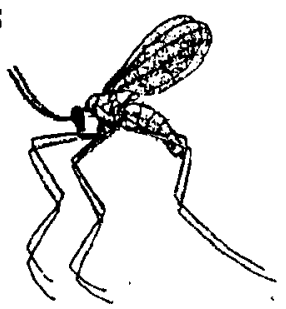

Aphelinus abdominalis: Beneficial Resources • Biobest • Duclos • Praxis - W. Neudorff

Aphidius colemani: (for cotton aphid): Biological Control • English Woodlands $\cdot$ IPM Labs $\cdot$ Koppert $\cdot$ Plant Sciences

Aphidius matricariae: Applied Bionomics $•$ Bunting $\cdot$ Beneficial Resources - FAR • Gartnerei - IPM Labs • Koppert - Natural Insect • Natural Pest Control $\cdot$ Nature's Alternative $\cdot$ Praxis $~ W$. Neudorff

Aphidius spp.: Biobest • Biological Crop • Chr. Hansens

Diaeretiella rapae: ARBICO - Praxis

Lysiphlebus testaceipes: Biological Control • Praxis

Praon volucre: English Woodlands

Trioxys pallidus(for walnut): Praxis

\section{Predators:}

Aphid midge, Aphidoletes aphidimyza: ARBICO - Applied Bionomics . Better Yicld • Biobest - Biological Crop • Bozeman - Budai • Bunting • Canadian Insectaries - Chr. Hansens • English Woodlands • FAR • Gartnerei • Hydro-Gardens • IPM Labs • Koppert • Natural Insect • Natural Pest Control - Nature's Control - Peaceful Valley - Plant Sciences - Praxis - Rincon-Vitova $\bullet$ Sautter \& Stepper - W. Neudorff Lacewings:

Chrysoperla carnea or Chrysopa carnea: Barmann - Bio Ag - BioAgronomics • Biological Crop • Biolab • BoBiotrol • Buena Biosystems - Bunting $\cdot$ Canadian Insectaries $\cdot$ Coast $\cdot$ Gardenville $\cdot$ Gartnerei $•$ Kuida $•$ Natural Insect $\cdot$ Natural Pest Controls $•$ Peaceful Valley $\bullet$ Praxis - Rincon-Vitova • Sauter \& Stepper - TriCal • Unique Insect Control • W. Neudorff

Chrysoperla comanche: Beneficial Resources $•$ Bio Ag • BioAgronomics $\cdot$ Coast $\cdot$ Kuida $\cdot$ Rincon-Vitova $\cdot$ TriCal

Chrysoperla rufilabris or Chrysopa rufilabris: ARBICO $\cdot$ Bio Ag $\cdot$ BioAgronomics - Biofac - Biological Pest B BoBiotrol - Bozeman - Buena Biosystems - Coast - IPM Labs - Kuida - M \& R Durango - Natural Insect • Nature's Control - Peaceful Valley $\cdot$ Praxis $\bullet$ Rincon-Vitova Sierra Ag $\cdot$ TriCal

Chrysoperla spp.: Beneficial Insectary • FAR • Kunafin • Snith \& Hawken Lady beetles:

Convergent lady beetle, Hippodamia convergens: ARBICO • Bio Ag •
Bio-Agronomics - Biofac • BoBiotrol - Bozeman - Coast - FAR • Gardenville • Hydro-Gardens - IPM Labs • Kuida • Kunafin • M \& R Durango - Natural Insect - Natural Pest Controls - Nature's Control • Peaceful Valley - Praxis - Rincon-Vitova • Smith \& Hawken • The Ladybug Company $\cdot$ TriCal $•$ Unique Insect Control

Lady beetle, Propylea quatuordecimpunctala: Praxis

\section{Predatory bugs:}

Mirid bug, Deraeocoris brevis: Applied Bionomics

Macrolophus caliginosus: Beneficial Resources

Orius albidipennis: Bunting - Koppen

Insidious flower bug, Orius insidiosis: Bunting - Gartnerei -IPM Labs -Koppert • Nature's Control

Orius laevigatus: Koppert

Orius spp.: Biobest $\cdot$ Biolab - Chr. Hansens - Duclos - Natural Insect Peaceful Valley $\cdot$ Plant Sciences

Minute pirate bug, Orius tristicolor: ARBICO • Bozeman - IPM Labs • Praxis

\section{Least-Toxic Chemical Controls}

\section{Diatomaceous Earth: See ANTS}

Insect Growth Regulators:

Sandoz Crop Protection (Enstar)

Insecticidal Oil:

Highly refined oil (from fish):

IFM - Peaceful Valley

Highly refined oll (from petroleum):

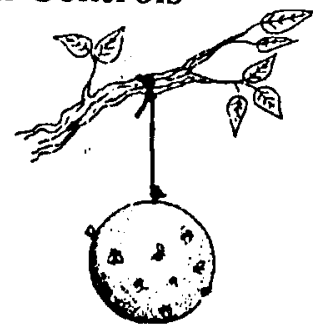

Monterey, Harmony (Saf-T-Side) • Valent USA, Harmony, Peaceful Valley (Volck) • Gardenville - Mycogen, Gardens Alive!, Harmony, IFM, Peaceful Valley (Sunspray)

Highly refined oil (from vegetables):

Peaceful Valley (Stoller Natur'l Oil) • ARBICO (Spreadall 93\% cottonseed)

\section{Insecticidal Soap:}

Mycogen, Harmony (M-Pede) • Agricola (ACT-92) • Necessary

(Concern)

With citrus oil:

Koppert(Savona) - Ringer, Bozeman, Gardens Alive!, Harmony, IFM, Nitron, Johnny's, Smith \& Hawken, Peaceful Valley, Charley's,

Gardenville (Safer) • ARBICO • Harmony • Necessary

With pyrethrin:

Necessary (Concern Multi-purpose)

With pyrethrum:

Ringer, Gardens Alive!, Harmony, Nitron, Gardenville (Safer Yard and Garden)

\section{Neem or azadirachtin:}

AgriDyne, Necessary, Harmony (Azatin) - Ringer, IFM, Harmony, Gardenville (BioNeem) • Grace-Sierra, Harmony (Margosan-O)

Pyrethrin:

Johnny's • Peaceful Valley (Pyrenone) • Roussel, Insects Limited (Kicker)

Pyrethrum:

Monterey (Bug-Buster-O) • Bozenan • Peaceful Valley • Gardenville

Repellent Garlic Spray:

Garlic Research Labs, Agape (Garlic Barrier) • Guardian Spray, New Earth (ENVIRepel, Guardian Spray) 
Ryania (restricted for use on commercial products):

Agri-Systems • Gardens Alive! • Harmony • IFM • Necessary • Peaceful Valley

\section{Sabadilla:}

Bozeman - Gardens Alive! • Gardenville - Harmony (Veratran) - IFM • Johnny's (Red Devil Dust) • Necessary (Sabadilla Pest Control) • Peaceful Valley

\section{BEES (See Yellowjackets)}

\section{BEETLES (Crop Pests)}

\section{Identification \& Monitoring}

Attractant for Cucumber Beetle:

Sigma Chemical, Peaceful Valley (eugenol)

Pheromones \& Traps:

For agricultural \& horticultural insects:

Ringer, Gardens Alive! (Safer Japanese Beetle Trap) • Pest Management, Great Lakes, Phero Tech(Multi-Pher, Unitrap, Delta Traps) Scentry, Pest Management, Great Lakes, Arbico, Bozeman (Scentry) • AgriSense (Decoy, Magnet) - Hercon, Consep, Great Lakes, Pest Management (Hercon Luretape) • Phero Tech (Phero Tech Lures) • Scentry, Great Lakes, Arbico, Bozeman, Pest Management, Natural Insect (Delta \& Wing Trap) • Trécé, Gardens Alive!, Great Lakes, Olson, Pest Management (Pherocon) • ARBICO • Harmony (Catch-em) - Consep, IFM, Harmony, Pest Management (Checkmate)

For forestry insects: Phero Tech (Phero Tech Lures)

For Japanese beetle: AgriSense - Trécé $\cdot$ Phero Tech For stored product insects: AgriSense, Great Lakes (Trappit ) - Consep, Insects Limited, Sarita Cruz Hort,

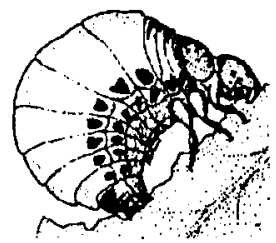
Arbico, Peaceful Valley, Great Lakes, Necessary, IFM, Pest Management (BioLure) • Insects Limited - Trécé, Pest Management (Storgard)

Vibratory Screens (to separate insects from grain): Eriez Magnetics Pty.

\section{Physical Controls}

Controlled Atmosphere System (for stored grains):

The Aerogen

Row Covers:

American Agrifabrics (Agryl) • Great Lakes (Reemay) - Gardener's Supply, Necessary, Gardens Alive! (Floating Row Covers) - Gardens Alive! (Super-Light Insect Barrier)• Harmony

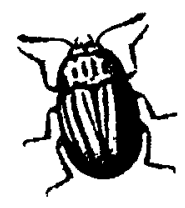
(Reemay, Tufbell) • Peaceful Valley (Reemay, Tufbell, Agryl, Poly)

Spiked lawn aerator sandals (for white grub control): Gardener's Supply • Bozeman

Sticky Tree Band:

Seabright, Great Lakes, Harmony, Phero Tech (Stickem Green)
Traps and Sticky Tape:

Sterling (Japanese Beetle Trap) • Phero Tech (Chroma) • Scentry, Great Lakes, Arbico, Bozeman, Pest Management (Scentry)

\section{Biological Controls}

Attractant for soldier bugs that attack pest beetles:

Sterling, Bozeman, Natural Insect (Rescuel)

Insect-Attacking Nematodes:

Heterorhabditis heliothidis: Gardens Alive!

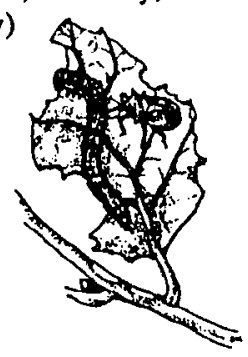

Heterorhabditis megidis: Biological Crop • De Groene

Heterorhabditis spp.: Better Yield - Bunting - English Woodlands •

Hydro-Gardens - IFM - Koppert $-M$ \& R Durango - Natural Insect • Nature's Control $\cdot$ Nematec $\cdot$ Praxis $\bullet W$. Neudorff

Steinernema bibionis: W. Neudorff

Steinernema carpocapsae or Neoaplectana carpocapsae: Biological

Crop - Biofac - BioLogic • Bioshield - Bozeman - Bunting • Ciba-Geigy

- De Groene- Gardens Alive! • Hydro-Gardens - IPM Labs • M \& R

Durango - Natural Insect $\cdot \mathrm{N}$-Viro $\cdot$ Peaceful Valley

Steinernema carpocapsae or Neoaplectana carpocapsae: M \& R Durango (Kapow strain)

Steinernema spp.: Better Yield - Biosys - Gardenville - English Woodlands • IFM • Johnny's • Koppert • Nature's Control • Nitron • Praxis

\section{Microbial Insecticides:}

Bacillus thuringiensis (BT):

Johnny's, Harmony (Colorado Potato Beetle Beater) - Sandoz Crop Protection (Trident) - Mycogen, IFM, Necessary, Natural Insect Control (M-Trak) - Ringer, Gardens Alive!, Peaceful Valley, Harmony (M-One)

\section{Bacillus thuringiensis tenebrionis (BTT):}

Novo Nordisk, Rol-Eko (Novodor)

Milky Spore Disease (Bacillus popilliae) (for Japanese beetle grubs): Fairfax, Arbico, Bozeman, Consep, Harmony, Necessary (Doom [BP], Japidemic [BP])

\section{Parasitoids:}

Anaphes spp. (for cereal leaf beetle): Praxis

Anisopteromalus calandrae (stored product beetles): ARBICO • Praxis Bathyplectes curculionis (for alfalfa weevil): Praxis

Cephalonomia waterstoni (for nusty grain beetle): Praxis

Edovum puttleri (for Mexican bean, CO potato beetle): Praxis

Lemophagus crioceritor (for asparagus beetle): Praxis

Meigenia mutabilis (for alfalfa weevil): Praxis

Microctonus spp. (for alfalfa weevil): Praxis

Pediobius foveolatus (for Mexican bean beetle): ARBICO - Praxis Rocky Mountain

Tetrastichus asparagi (for asparagus beetle): Praxis Tetrastichus julis (for cereal leaf beetle): Praxis

Predators:

Predatory stink bug, Perillus bioculatus (for Colorado potato beetle): Praxis
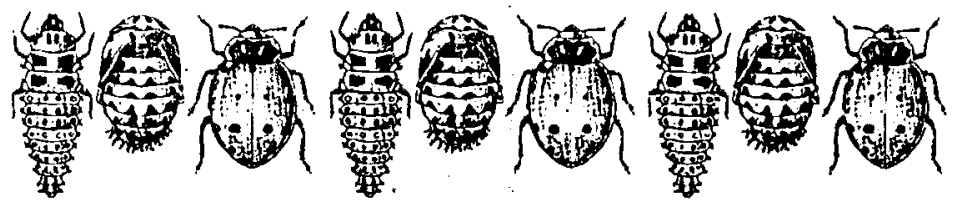
Spined soldier bug, Podisus maculiventris (for CO potato beetle): ARBICO • Gardens Alive! • Natural Insect • Praxis

Warchouse pirate bug, Xylocoris flavipes (for stored product beetles): ARBICO • Biofac • Praxis

\section{Least-Toxic Chemical Controls}

Baits (cucurbitacin, with carbaryl for cucumber beetle): MicroFlo (Adios)

Borate-Based Insecticides:

Whitmire (PT 240 or PT 249 Perma-Dust)

Cryolite:

Peaceful Valley (Cryolite 96) • Monterey (Kryocide)

Diatomaceous Earth: See ANTS

Insect Growth Regulators:

Fenoxycarb:

Ciba Geigy, Target (Torus system)

Insecticidal Oil: See APHIDS

Insecticidal Soap: See APHIDS

Neem or azadirachtin:

AgriDyne, Necessary, Harmony (Azatin) - Ringer, IFM, Harmony, Gardenville (BioNecm) - AgriDyne (Align) • Grace-Sierra, Harmony (Margosan-O)

Pyrethrin:

Roxide - Whitmire (PT 3-6-10 Aero-Cide, PT-170 X-Clude, PT-175 MicroCare) ·Johnny's (Pyrenone) • Hess \& Clark (Capsul-Ban) • Roussel, Insects Limited (Kicker)

Ryania (restricted for use on commercial products):

Agri-Systems • Gardens Alivel • Harmony • IFM • Necessary • Peaceful Vallcy

Sabadilla:

Bozeman - Gardens Alivel • Gardenville - Harmony (Veratran) - IFM • Johnny's (Red Devil Dust) - Necessary (Sabadilla Pest Control) . Peaceful Valley

Silica Aerogel: See ANTS

\section{BEETLES (Structural Pests)}

\section{Identification \& Monitoring}

Trained Dogs (for detection):

Industrial Narcotic • TADD (TADD Dogs)

\section{Physical Controls}

Electrical Treatment

(for drywood termite \& boring beetles)

ETEX (Electrogun)

Heat Treatment:

Mission Pest Control

- Isothermics (Call for

local practitioners) - Steffenson (Northern CA only)

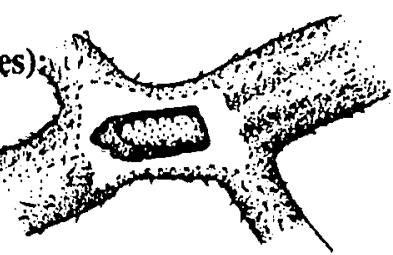

Microwave Treatment:

The Termite Inspector

\section{Least-Toxic Chemical Controls}

Borate Rods:

CSI, NISUS (Impel Rods)

Borate Termiticide \& Wood Preservative:

NISUS (Bora-Care) - U.S. Borax (TimBor)

\section{BLACKFLY (See Mosquitoes)}

\section{BORERS (See Beetles)}

\section{BUGS (Hemipterans)}

\section{Identification \& Monitoring}

Pheromones \& Traps:

Trécé, Gardens Alive!, Great Lakes, Olson, Pest Management (Pherocon)

\section{Physical Controls}

Row Covers:

American Agrifabrics (Agryl)

- Great Lakes (Reemay) - Gardens Alive! (Super-Light) - Gardener's

Supply, Gardens Alive!, Necessary (Floating Row Covers) • Harmony

(Reemay, Tufbell) • Peaceful Valley (Reemay, Tufbell, Agryl, Poly)

Screening:

ATI (Greenhouse screen) • Tie-Net

Vacuum:

BioQuip • D-VAC

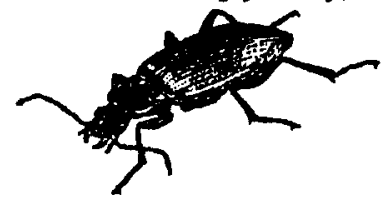

\section{Horticultural Controls}

Insect-Resistant Turfgrass:

Endophytic Ryegrass (repels blllbugs \& caterpillars):

Lofts Seed (Repell) - Turf Seed (Citation II, Birdic II, Omega II, CBS II, Manhattan II, Charger, Quickstart, 246 Sunrye) - Turf Seed (Sunrye) Endophytic tall fescue:

Turf Sced (Apache)

\section{Biological Controls}

Attractant for soldier bugs that attack pest bugs:

Sterling, Bozeman, Natural Insect (Rescue!)

Microbial Insecticides:

Entomopathogenic fungus:

Metarhizium anisopliae (for meadow spittlebug, coffee leafminer): Agricola

Parasitoids:

Trissolcus spp. (for stink bug eggs): Praxis

Predators:

spined soldier bug. Podisus maculiventris

(for $\mathrm{CO}$ potato beetle, bugs general,

caterpillar general): ARBICO -

Gardens Alive! • Praxis • Natural Insect

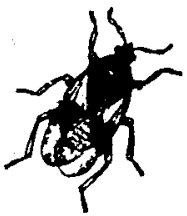




\section{Least-Toxic Chemical Controls}

Borate-Based Insecticides:

Whitmire (PT 240 or PT 249 Perma-Dust)

Diatomaceous Earth: See ANTS

Insecticidal Soap: See APHIDS

Neem or azadirachtin:

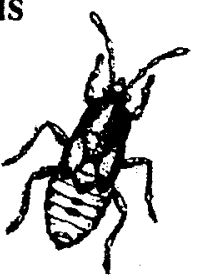

AgriDyne, Necessary, Harmony (Azatin) • Ringer, IFM, Harmony, Gardenville (BioNeem) • Grace-Sierra, Harmony (Margosan-O)

Pyrethrin:

Whitmire (PT 3-6-10 Aero-Cide, PT-565 Plus) - Johnny's, Peaceful

Valley (Pyrenone) • Hess \& Clark (Capsul-Ban) • Roussel, Insects Limited (Kicker)

Ryania (restricted for use on commercial products):

Agri-Systems • Gardens Alive! • Harmony • IFM • Necessary • Peaceful Valley

Sabadilla:

Bozeman - Gardens Alive! - Gardenville - Harmony (Veratran) - IFM • Johnny's (Red Devil Dust) • Necessary (Sabadilla) • Peaceful Valley

Silica Aerogel: See ANTS

\section{CATERPILLARS}

\section{Identification \& Monitoring}

Apple scab forecasting equipment:

Pest Management (PSAOC consultant software)

Pheromones \& Traps:

For agricultural \& horticultural insects:

Great Lakes, Pest Management (Multi-Pher, Unitrap, Delta Traps) • Phero Tech, Great Lakes (Unitrap) - Scentry, Pest Management, Great Lakes, Arbico, Bozeman (Scentry) • AgriSense (Decoy, Magnet) • Consep (SureFire) - Hercon, Consep, Great Lakes, Pest Management (Hercon Luretape) - Phero Tech (Phero Tech Lures) - Scentry, Great Lakes, Arbico, Bozeman, Pest Management, Natural Insect (Delta \& Wing Trap, Heliothis Trap) - Trécé, Gardens Alivel, Great Lakes, Olson, Pest Management (Pherocon) - ARBICO, Harmony (Catch-em) • Peaceful Valley (Zoecon) - Consep, IFM, Harmony, Pest Management (Checkmate)

For forestry insects:

Phero Tech (Phero Tech Lures)

For stored product insects:

Consep, Insects Limited, Santa Cruz Hort, Arbico, Peaceful Valley, Great Lakes, Necessary, IFM, Pest Management (BioLure)- Phero Tech (Phero Tech Lures) • Trécé, Pest Management (Storgard)

\section{Physical Controls}

Controlled Atmosphere System.(for stored grains):

The Aerogen

Row Covers:

American Agrifabrics (Agryl) • Great Lakes (Reemay) • Gardens Alive! (Super-Light) • Gardener's Supply, Gardens Alivel, Necessary (Floating Row Covers) - Harmony (Reemay, Tufbell) • Peaceful Valley (Reemay, Tufbell, Agryl, Poly)

Sticky Barrier:

Intagra (Bug Gum) • Tanglefoot, Bozeman, Gardens Alive!, Great
Lakes, Harmony, Necessary, Phero Tech, Gardenville (Tanglefoot)

Sticky Trap/Tape:

For stored product insects:

Santa Cruz Hort (Wing \& Delta Traps)

For agricultural $\&$ hortlcultural insects:

Scentry, Great Lakes, Arbico, Bozeman, Pest Management (Scentry Gypsy Moth Trap)

Traps:

Consep (Gypsy Moth Trap) - Ladd, Bozeman (Red Sphere Fruit Fly) • Consep, Harmony (SureFire Codling Moth)

Tree Wrap:

Olson (Stiky Tree Guards) • Bozeman, Peaceful Valley (Bug Belt) • Great Lakes (Tred Not Tape) - Intagra (Tree Banding Tape) - Seabright, Great Lakes, Harmony, Phero Tech (Stickem Green)

\section{Horticulturál Controls}

Insect-Resistant Turfgrass:

Endophytic Ryegrass (repels billbugs \& caterplllars):

Lofts Seed (Repell) - Turf Seed (Citation II, Birdie II, Omega II, CBS II, Manhattan II, Charger, Quickstart, 246 Sunrye, Sunrye)

Endophytic tall fescue:

Turf Seed (Apache)

\section{Biological Controls}

Attractant for soldier bugs that attack pest caterpillars:

Sterling, Bozeman, Natural Insect (Rescue!)

Insect-Attacking Nematodes:

Steinernema carpocapsae: BioLogic, IPM Labs, Natural Insect Control (Scanmask, Hort Scanmask)

Microbial Feeding Stimulant:

Harmony (Entice) • Sandoz Crop Protection (Gustol)

Microbial Insecticides:

Bacillus thuringiensis (BT):

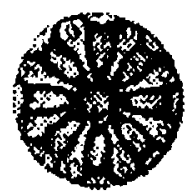

Abbott, Peaceful Valley, Johnny's, English Woodlands (Dipel, Dipel 4L, 2X, 10G, ES, Xentari WDG) - Insects Limited (Topside Dipel) •

Koppert, PBI/Gordon (Bactospeine) • Nitron, Gardenville (Caterpillar Killer) - ARBICO - IFM - Necessary - Bozeman - Ciba Geigy (Agree, Design) - Agricola (BST-88, BST-88A)

\section{Bacillus thuringiensis kurstaki (BTK):}

Ecogen (Cutlass) - Monterey (Lepid BT) • Mycogen (M-Peril) • Mycogen, Gardens Alive! (MVP) • Novo Nordisk, Rol-Eko (Biobit, Foray) • Ringer, Peaceful Valley, Harmony, Gardenville (Caterpillar Attack, Vegetable Insect Attack) - Sandoz Crop Protection, Peaceful Valley, Harmony (Javelin WG) • Bozeman - Monterey, Harmony (Worm-Ender)

Codling moth granulosis virus:

Association for Sensible Pest Control (For experimental use only) NPV (nucleopolyhedrosis virus) (for codling moth): Agricola (VPN-80, VPN-82) - U.S. Forest Service, USDA

Parasitoids:

Bracon hebetor (for stored product moth): Biofac $\cdot$ Praxis

Casinaria arjuna (for gypsy moth): Praxis

Coccygomimus disparis (for gypsy moth): Praxis

Cotesia marginiventris (for gypsy moth, stored product moth): ARBICO

- Biofac 
Cotesia melanoscela (Korean strain) (for gypsy moth): BioBase - Praxis Cotesia plutellae (for diamondback moth): ARBICO • Biofac Diadegma insularis (for diamondback moth): ARBICO - Praxis Glyptapanteles flavicoxis (for gypsy moth): BioBase

Glyptapanteles indiensis (for gypsy moth): Praxis

Goniozus legneri (for navel orangeworm, omnivorous leafroller):

ARBICO - Bio Ag - Bio-Agronomics - Biological Pest - BoBiotrol •

Coast - FAR - Kuida - Peaceful Valley - Praxis - Rincon-Vitova - TriCal

Lydella thompsoni (for European corn borer): Praxis

Macrocentrus ancylivorus (for Oriental fruit moth): ARBICO - Praxis

Meteorus pulchricornis (for gypsy moth): Praxis - BioBase

Microchelonus blackburni (for pink bollworm): Praxis

Microplitis plutellae (for diamondback moth): Praxis

Ooencyrtus kuvanae (for gypsy moth): Praxis

Pentalitomastix plethoricus (for navel orangeworm): ARBICO •

BoBiotrol - Praxis

Trichogramma dendrolimi (for moth eggs): BoBiotrol

- Trichogramma evanescens (for moth): Biobest

Trichogramma georgia (for moth eggs in field crops): Buena Biosystems

Trichogramma minufum (for Eastern U.S. orchards): ARBICO - Biofac • BoBiotrol - Bozeman - Buena Biosystems - FAR - Gardens Alive! - M \& R Durango - Nature's Control

Trichogramma planeri (for Westem U.S. orchards): ARBICO - Bio Ag - Bio-Agronomics - Biological Pest - BoBiotrol - Bozeman - Buena Biosystems - Coast - FAR - Kuida - Rincon-Vitova - TriCal

Trichogramma pretiosum (for moth eggs in vegetable \& field crops): ARBICO - Bio Ag - Bio-Agronomics - Biofac - Biological Pest BoBiotrol - Bozeman - Buena Biosystems - Coast - FAR - Gardens Alive! - Kuida - M \& R Durango - Nature's Control - Rincon-Vitova - TriCal

Trichogramma spp. (for moth eggs in vegetable \& field crops): Beneficial Insectary - Conrad Appel - Gardenville - KWS - Kunafin - Natural Insect• Natural Pest Controls - Peaceful Valley - Praxis - Smith \& Hawken

Trichogrammatoidea bactrae (for moth egg in fields \& orchards, pink bollworm): Praxis - Rincon-Vitova

\section{Predators:}

Spined soldier bug, Podisus maculiventris: ARBICO - Gardens Alive! • Natural Insect - Praxis

Warehouse pirate bug, Xylocoris flavipes: (for stored product moths): ARBICO - Biofac - Praxis

\section{Least-Toxic Chemical Controls}

Cryolite:

Peaceful Valley (Cryolite 96) • Monterey (Kryocide)

\section{Diatomaceous Earth: See ANTS}

Insect Growth Regulators:

Fenoxycarb:

Ciba Geigy, Target (Torus system)

\section{Insecticidal Oil: See APHIDS}

\section{Insecticidal Soap: See APHIDS}

Mating Confusants:

Consep, IFM, Harmony (Checkmate) - Pacific Biocontrol • Great Lakes, Harmony, Necessary, Peaceful Valley, IFM (ISOMATE) - Scentry, Great Lakes, Arbico, Bozeman (NoMate for tomato pinworm) - Scentry, Great Lakes, Arbico, Bozeman, Pest Management (Attract \& Kill,
NoMate PBW-MEC for pink bollworm)

Neem or azadirachtin:

AgriDyne, Necessary, Harmony (Azatin) - Ringer, IFM, Harmony, Gardenville (BioNeem) - AgriDyne (Align) - Grace-Sierra, Harmony
(Margosan-O).

Pyrethrin:

Roxide - Whitmire (PT 3-6-10 Aero-Cide) - Waterbury (Purge III) Johnny's, Peaceful Valley (Pyrenone) - Hess \& Clark (Capsul-Ban) • Roussel, Insects Limited (Kicker)

Pyrethrum:

Monterey (Bug-Buster-O)

Ryania (restricted for use on commercial products):

Agri-Systems • Gardens Alive! • Harmony - IFM • Necessary • Peaceful Valley

\section{Sabadilla:}

Bozeman - Gardens Alive! - Gardenville - Harmony (Veratran) - IFM Jolınny's (Red Devil Dust) - Necessary (Sabadilla Pest Control) • Peaceful Valley

Silica Aerogel: See ANTS

\section{COCKROACHES}

\section{Identification \& Monitoring -}

Flashlight with Red Filters:

Streamlight, Brody (Streamlight)

Pheromones \& Traps:

GTF Labs (Rid-A-Roach) • Bi-Pro Industries (GP-1 Pheromone Lure)

\section{Physical Controls}

Caulk:

Whitmire, Brody (Pest Proof Caulk)

Traps:

AgriSense, Great Lakes (Trappit) • Bi-Pro Industries (ZAP-TRAP)

Glue board trap:

Bell (Trapper) - Bi-Pro Industries (EX1GT Glue Tray)

Trap \& attractant:

Consep (The Dungeon) • Brody (Roach Pot) - Seabright (Safe Stop)

Vacuums:

Rexair (Rainbow Water Vacuum)

\section{Biological Controls}

Microbial Insecticides:

Metarhizium fungal bait station:

EcoScience (Biopath)

Parasitoids:

Anastatus tenviles: Praxis

Comperia merceti: Praxis

Tetrastichus hagenowii: Praxis

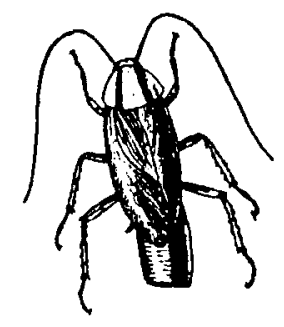

\section{Least-Toxic Chemical Controls}

Baits:

Abamectin: 
Whitmire (Avert PT-300, PT-310) • Gardens Alive!

Borate-based:

NISUS (NIBAN Granular Bait) • Blue Diamond Exterminating (Blue

Diamond) - Seabright (Roach Free)

Hydramethylnon (child-resistant):

Clorox Company (Maxforce, Combat)

Borate-Based Insecticides:

Organic Control (Ant-T-Roach) • Enforcer, Gardenville (Roach Ridd) • Waterbury (Mop-Up, Roach-Kil, Borid) • Whitmire (PT 240 or 249

Perma-Dust) • Copper Brite, Harmony (Roach Prufe) - Perma Proof • Peaceful Valley

Diatomaceous Earth:

Natural Insecto (Insecto) • Organic Plus • Necessary

With pyrethrin:

Diacide (Homeguard) • Permaguard, Pristine, Nitron, Gardenville

(Permaguard) - Organic Plus

Fumigation bags:

Urban Insect Solutions

Hydramethylnon:

Cyanamid, Agricultural Products Division (Siege)

Insect Growth Regulators:

Fenoxycarb:

Ciba/Geigy, Target (Torus system)

Hydroprene:

Zoecon, Gardenville (Gentrol)

Insecticidal Soap, with citrus oil:

Ringer, Gardens Alive!, Peaceful Valley, Gardenville (Safer)

Pyrethrin:

Roxide - Whitmire (PT 3-6-10 Aero-Cide, PT-170 X-Clude, PT-175

MicroCare, PT-565 Plus) • Hess \& Clark (Capsul-Ban) - Roussel,

Insects Limited (Kicker)

Ryania (restricted for use on commercial products):

Agri-Systems (Natur-Gro Roach Powder)

Silica Aerogel:

100\% Pure:

Cabot (Cab-O-Sil) (Must order 10 pound minimum) $・$ Degussa

(Wessalon S., Aerosil 380) (Must order 25 pound minimum)

With ammonium fluosilicate:

Roussel (Dri-Die; 95\% silica aerogel, 2\% ammonium fluosilicate; 3\% inerts) - Target (Dri-Die)

With pyrethrin:

Purity Products - Roussel, Insects Limited,

Target, Gardenville (Drione) • Roxide,

Harmony (Revenge) •

Whitmire (PT 230 or PT 239 Tri-Die)•

Van Waters \& Rogers

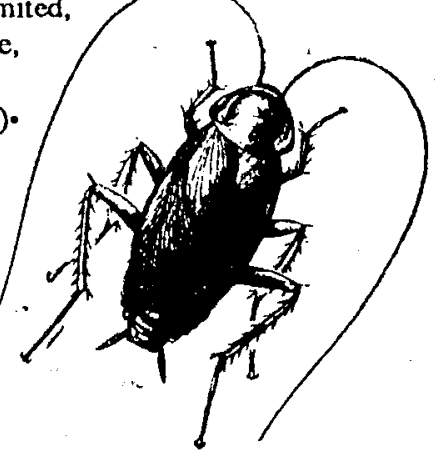

\section{CRICKETS (See Grasshoppers)}

\section{EARWIGS}

\section{Physical Controls}

Barriers \& Exclusion Devices:

Intagra (Tree Banding Tape, Bug Gum)

Caulk:

Whitmire, Brody (Pest Proof Caulk)

\section{Least-Toxic Chemical Controls}

Diatomaceous Earth: See ANTS

Insecticidal Soap: See APHIDS

\section{FLEAS}

\section{Identification \& Monitoring}

Sticky light trap:

Enforcer Products (Over Nite Flea Trap)

\section{Physical Controls}

\section{Combs:}

Gardens Alive! - Hansen's Pet (Ancol)

- Hogil (Innomed)

Vacuums:

Rexair (Rainbow Water Vacuum)

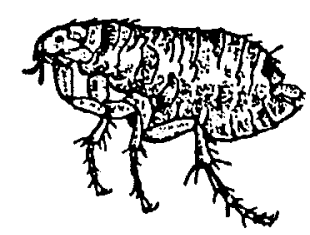

\section{Biological Controls}

Insect-Attacking Nematodes:

Biosys (Vector for licensed PCO's only, BioFlea)

\section{Least-Toxic Chemical Controls}

Boric acid powder formulation:

Flea Busters (Rx for Fleas) (Call company for treatment)

Diatomaceous Earth:

EcoSafe Products (EcoŹne) - Natural Insecto (Insecto) • Organic Plus • Necessary - Permaguard

With pyrethrin:

Diacide (Homeguard) • Permaguard, Pristine, Nitron (Permaguard) •

Organic Plus

Insect Growth Regulators:

Methoprene (with permethrin) for fleas:

Zoecon, Gardens Alive!, Necessary, Peaceful Valley (Vigren, Flea'Trol) • Enforcer (Flea Fogger, Flea Spray)

Insecticidal Oil:

Citrus oll (d-limonene) extract:

Farnam, Gardens Alive! (Flea Stop Shampoo) - Vet Express (VIP Pet

Spray, Shampoo, Dip) - Hansen's Pet (Citrus Plus Shampoo, Spray)

Insecticidal Soap:

With citrus oil:

Ringer, Bozeman, Gardenville (Safer Flea Soap, Spray) 
With citrus oll \& pyrethrum:

Harmony, Bozeman, Gardens Alive! (Flea \& Tick Attack)

With pyrethrum:

Ringer, Bozeman, Gardens Alive!, Harmony (Flea \& Tick Attack) •

Ringer, Gardens Alivel, Harmony, Nitron, Gardenville (Safer Yard and

Garden)

\section{Pyrethrin:}

Del (Lice, Tick \& Flea Spray) • Roxide • Whitmire (PT 3-6-10 Aero-

Cide, PT-175 MicroCare, PT-565 Plus) - Enforcer (Flea \& Tick Spray,

Powder, Shampoo) - Hess \& Clark (Capsul-Ban) - Roussel, Insects

Limited (Kicker)

Pyrethroid (microencapsulated):

3M Corp (Sectrol) (Sold only to veterinarians)

Pyrethrum:

EcoSafe Products, Gardenville (EcoZone)

Silica Aerogel: See ANTS

\section{FLIES (Nuisance)}

\section{Identification \& Monitoring}

Pheromones \& Traps:

AgriSense (Decoy, Magnet) ; AgriSense, Great Lakes, Pest Management (Trappit) • Farnam (Trap 'n Toss) • Phero Tech (Phero Tech Lures) • Phero Tech, Great Lakes (Unitrap) - Trécé, Gardens Alivel, Great Lakes, Olson, Pest Management (Pherocon)

\section{Physical Controls}

Fly bonnet for horses:

WF Young (Fly Bonnet)

Screening:

ATI (Greenhouse screen)

- Hydro-Gardens (Fly Barr) • Tie-Net

Sticky Adhesive:

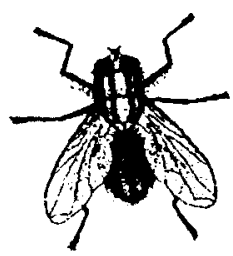

Tanglefoot, Arbico, Bozeman, Great Lakes,

Pest Management, Gardenville, Harmony, Natural Insect Control,

Charley's (Tangle-Trap)

Traps:

ARBICO (Solar) • Baley's (Pyramid) • Great Lakes (Musca Terminator)

- Olson (Stiky) • Roxide (Aeroxon) • Sterling (Rescue!) • Woodstream,

Brody, Great Lakes, Peaceful Valley, Natural Insect Control (Victor FlyIn-Saucer)

Light traps:

D. Gilbert Industries (Electrocuting Light Traps)

Solar ny traps:

ARBICO $\cdot$ Natural Insect

Sticky trap/tape:

Hess \& Clark (Sticky Roll) • Olson, Great Lakes, Pest Management

(Stiky Stuff, Stiky Traps) • Phero Tech (Chroma)

\section{Biological Controls}

Insect-Attacking Nematodes:

Heterorhabditis heliothidis: Gardens Alive!

Heterorhabditis megidis: Biological Crop • De Groene
Heterorhabditis spp.: Better Yield - Bunting - Hydro-Gardens - IFM -

Koppert • M \& R Durango - Natural Insect - Nature's Control - Nematec

- Praxis $\bullet W$. Neudorff

Steinernema bibionis: W. Neudorff

Steinernema carpocapsae or Neoaplectana carpocapsae: Biofac .

BioLogic - Biological Crop - Bozeman - Bunting - Ciba-Geigy - De

Groene - Gardens Alive! $\cdot$ Hydro-Gardens $\cdot$ IPM Labs $\cdot M$ \& R Durango

- Natural Insect • N-Viro - Peaceful Valley

Steinernema spp.: Better Yield • Biosys • Gardenville • IFM • Johnny's - Koppert • Nature's Control $\cdot$ Nitron $\cdot$ Praxis

\section{Microbial Insecticides:}

Ivermectin for bot flies, etc.:

Merck Ag Vet(Ivomec Pour-On) (Sold only to veterinarians \& licensed suppliers)

Ivermectin and clorsulon:

Merck Ag Vet(Ivomec-F Injection) (Sold only to veterinarians \& licensed suppliers)

Parasitoids:

Muscidifurax raptor: Biofac $\cdot$ Gardenville $\cdot$ IPM Labs $\cdot$ Kunafin

Muscidifurax raptorellus: Buena Biosystems

Muscidifurax spp.: Beneficial Insectary • BoBiotrol - Peaceful Valley • Praxis - Spalding

Muscidifurax zaraptor: ARBICO - Bozeman - Canadian Insectaries • Kunafin $\cdot M$ \& R Durango $\cdot$ Natural Pest $\cdot$ Rincon-Vitova • Urbio

Nasonia vitripennis or Mormoniella vitripennis: ARBICO - Natural Pest - Praxis • Urbio

Pachycrepoideus vindemiae: Kunafin - Praxis

Spalangia cameroni: Kunafin

Spalangia corana: Biofac

Spalangia endius: ARBICO • Canadian Insectaries • Kunafin • Natural

Pest $\cdot$ Rincon-Vitova $\cdot$ Urbio

Spalangia nigroaenea: Kunafin $\cdot$ Rincon-Vitova

Spalangia spp.: Beneficial Insectary • Buena Biosystems • M \& R

Durango - Peaceful Valley $\cdot$ Praxis $\cdot$ Spalding

Sphegigaster sp.: Peaceful Valley • Praxis

Urolepis rufipes: Praxis

Parasitic mites:

Glyptholaspis confusa: Praxis

\section{Predators:}

Histerid beetle, Carcinops pumilio: Praxis

Predatory mite, Macrocheles spp.: Praxis

Dung beetle, Onthophagus gazella: Praxis

Predatory fly, Ophyra aenescens: Praxis

\section{Least-Toxic Chemical Controls}

\section{Diatomaceous Earth: See ANTS}

Pyrethrin:

Roxide $•$ Roxide, Harmony (Revenge) • Whitmire (PT 3-6-10 Aero-

Cide, PT-175 MicroCare, PT-565 Plus) • Waterbury (Purge III) - Hess \& Clark (Capsul-Ban) • Roussel, Insects Limited (Kicker)

\section{Repellents:}

Bozeman, Natural Insect Control, Gardenville (Skeeter Shoo, Botanical)

Silica aerogel: See ANTS 


\section{Identification \& Monitoring}

Pheromones \& Traps:

ARBICO, Harmony (Catch-em) - Hercon, Great Lakes, Pest Management (Multi-Pher, Unitrap, Delta) • Trécé, Gardens Alive!, Great Lakes, Olson, Pest Management (Pherocon) - Scentry, Pest Management, Great Lakes, Arbico, Bozeman (Scentry Gypsy Moth)

\section{Physical Controls}

Traps:

Apple maggot red sphere trap:

Ladd, Pest Management, Harmony, Necessary, Natural Insect,

Gardenville (Ladd) • Consep, Harmony (SureFire)

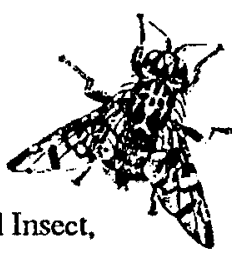

Light traps:

D. Gilbert Industries (Gilbert Insect Light Traps)

Sticky trap/tape:

Phero Tech (Chroma) - Scentry, Great Lakes, Arbico, Bozeman, Pest Management (Jackson Fruit Fly Trap) • Olson

\section{Least-Toxic Chemical Controls}

Pyrethrin:

Roussel, Insects Limited (Kicker)

\section{FUNGUS GNATS}

\section{Physical Controls}

Screening:

Hydro-Gardens (Fly Barr) • ATI (Greenhouse screen) • Tie-Net

Sticky Adhesive:

Seabright, Great Lakes, Harmony, Phero Tech (Stickem Special) • Tanglefoot, Arbico, Bozeman, Great Lakes, Pest Management, Gardenville, Harmony, Natural Insect, Charley's (Tangle-Trap)

Traps:

Arbico - Gardenville $\cdot$ Hansens Bio Systems - Natural Insect $\cdot$ Ringer, Gardenville (Safer Flying Insect) • Phero Tech (Chroma) • Olson, Great Lakes, Pest Management (Stiky Stuff, Stiky Traps)

\section{Biological Controls}

Insect-Attacking Nematodes:

Heterorhabditis heliothidis: Gardens Alive!

Heterorhabditis megidis: Biological Crop • De Groene

Heterorhabditis spp.: Better Yield - Bunting - Hydro-Gardens - IFM Koppert - M \& R Durango - Nature's Control $\cdot$ Natural Insect $\cdot$ Nematec - Praxis • W. Neudorff

Steinernema bibionis: W. Neudorff

Steinernema carpocapsae or Neoaplectana carpocapsae: Biofac • BioLogic - Biological Crop - Bioshield • Bozeman - Bunting • CibaGeigy - De Groene - Gardens Alive! • Hydro-Gardens $• M$ \& R Durango - N-Viro • Peaceful Valley

Steinernema spp.: Better Yield $\cdot$ Biosys $\cdot$ Gardenville $\cdot$ Hydro-Gardens • IFM - Johnny's • Koppert • Nature's Control • Nitron - Praxis
Microbial Insecticides:

Bacillus thuringiensis (BT):

Abbott, Gardens Alive!, Harmony, IPM Labs, Peaceful Valley, Gardenville (Gnatrol)

Predators:

Predatory mite, Geolaelaps spp.: Praxis • Rincon-Vitova

Predatory mite, Hypoaspis miles: ARBICO • Applied Bionomics • IPM

Labs - Natural Insect

\section{Least-Toxic Chemical Controls}

\section{Diatomaceous Earth: See ANTS}

Insect Growth Regulators:

Sandoz Crop Protection (Enstar)

Methoprene:

Zoecon (Apex) • Sandoz (Enstar)

Insecticidal Oil: See APHIDS

Neem or azadirachtin:

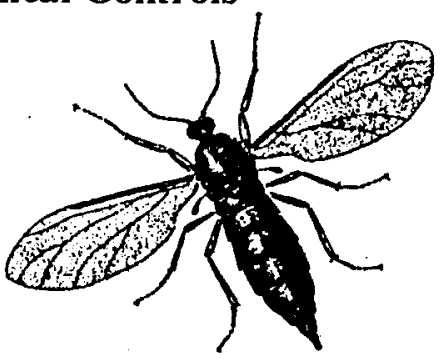

AgriDyne, Necessary, Harmony (Azatin) - Ringer, IFM, Harmony, Gardenville.(BioNeem) • Grace-Sierra, Harmony (Margosan-O)

Pyrethrin:

Hess \& Clark (Capsul-Ban) • Whitmire (PT 3-6-10 Aero-Cide, PT-565 Plus)

\section{GRASSHOPPERS}

\section{Physical Controls}

\section{Row Covers:}

American Agrifabrics (Agryl) • Great Lakes (Reemay) - Gardener's Supply, Gardens Alive!, Necessary (Floating Row Covers) • Gardens Alive! (Super-Light) • Harmony (Reemay, Tufbell) • Peaceful Valley (Reemay, Tufbell, Agryl, Poly)

\section{Biological Controls}

Microbial Insecticides:

Protozoan pathogen, Nosema locustae:

Gardenville $\cdot$ Harmony $\cdot M$ \& R Durango $\cdot$ Nitron, Bozeman, Gardens Alive! (Semispore Bait)

\section{Least-Toxic Chemical Controls}

Borate-Based Insecticides:

NISUS (NIBAN Granular Bait for crickets) • Whitmire (PT 240 or PT 249 Perma-Dust)

\section{Diatomaceous Earth: See ANTS}

\section{Insecticidal Soap: See APIIDS}

Repellent Garlic Spray:

Guardian Spray, New Earth (ENVIRepel, Guardian Spray) • Garlic Research Labs, Agape (Garlic Barrier)

Ryania (restricted for use on commmercial products): Agri-Systems • Gardens Alive! • Harmony - IFM - Necessary • Peaceful Valley

Sabadilla:

Bozeman • Gardens Alive! • Gardenville • Harmony (Veratran) • IFM 


\section{GRUBS (See Beetles)}

\section{LEAFHOPPERS}

\section{Identification \& Monitoring}

Sticky trap/tape:

Scentry, Great Lakes, Arbico, Bozeman (Versagard)

\section{Physical Controls}

Barriers \& Exclusion Devices:

Bozeman - Gardens Alive!

Plastic film to attract leafhoppers:

Entosphere (HopperFinder)

Sticky Adhesive:

Seabright, Great Lakes, Harmony, Phero Tech (Stickem Special)

Traps and Sticky Tape:

Bozeman - Gardens Alivel - Harmony - IFM - IPM Labs - Ringer, Gardenville (Safer) • Necessary • Phero Tech (Chroma)

\section{Biological Controls}

Parasitoids:

Anagrus atomus? English Woodlands

Anagrus epos (for grape leafhopper): ARBICO • Praxis

Anagrus spp.: Biological Crop

\section{Least-Toxic Chemical Controls}

\author{
See APHIDS
}

\section{LEAFMINERS}

\section{Physical Controls}

Sticky Trap/Tape:

Olson, Pest Management

\section{Biological Controls}

Microbial Insecticides:

Entomopathogenic Fungus:

Metarhizium anisopliae (for meadow spittlebug, còffee leafminer):

Agricola

Parasitoids:

Dacnusa sibirica: ARBICO - Beneficial Resources - Biobest - Biofac • Bunting - Chr. Hansens - Duclos - Gartnerei - Koppert - Natural Pest Control - Natural Pest Controls - Praxis • Sautter \& Stepper • W.

Neudorff
Dacnusa spp.: Biological Crop

Diglyphus isaea: - ARBICO - Azienda - Biobest - Biofac - Biolab •

Biological Control - Bunting - Chr. Hansens - Duclos - Gartnerci •

Koppert - Natural Pest Control - Natural Pest Controls - Plant Sciences •

Sautter \& Stepper - W. Neudorff

Diglyphus spp.: Beneficial Resources • Biological Crop • Praxis

\section{Least-Toxic Chemical Controls}

Neem or azadirachtin:

AgriDyne, Necessary, Harmony (Azatin) - Ringer, IFM, Harmony, Gardenville (BioNeem) • Grace-Sierra, Harmony (Margosan-O)

\section{LICE}

\section{Identification \& Monitoring}

Head lice detection kit:

Del (Pronto Lice Detection Kit, for home use)

Head lice prevention, management and educational products:

National Pediculosis Association

\section{Physical Controls}

Head lice comb:

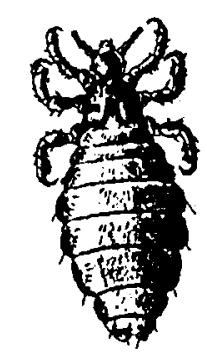

Health Enterprises (MC-12) • Hogil (Innomed)

\section{Least-Toxic Chemical Controls}

Pyrethrin:

Del (Bare Liquid, Lice, Tick \& Flea Spray)

Pyrethrin shampoo, for head lice:

Carter-Wallace (Triple X) • Del (Pronto) • Pfizer (RID) • Smith Kline

Beecham (A-200) (For large retailers only)

Pyrethrin, with pubic lice comb:

Hogil (Innogel Plus for pubic lice)

\section{MAGGOTS (See Flies)}

\section{MEALYBUGS}

\section{Physical Controls}

Screening:

ATI (Greenhouse screen) • Hydro-Gardens (Fly Barr) • Tie-Net

\section{Biological Controls}

Parasitoids:

Anagyrus pseudococci: Amos Rubin - Praxis

Leptomastidea abnormis: Amos Rubin - Praxis

Leptomastix dactylopii: Amos Rubin • Azienda • Biolab • Biological Control • Bugs for Bugs • Canadian Insectarics • IPM Labs • Natural Insect $\cdot$ Praxis $\cdot$ W. Neudorff 
Leptomastix sp.: J.P. Kass

Pauridia peregrina: Praxis

Pseudaphycus maculipennis: J.P. Kass

\section{Predators:}

Lacewing, Sympherobius sanctus: Amos Rubin

Lady beetles:

Mealybug destroyer, Cryptolaemus montrouzieri: ARBICO - Amos Rubin - Azienda - Biobest - Biofac - Biolab - Biological Control Biological Crop - Bozeman - Bugs for Bugs • Bunting - Canadian Insectaries - English Woodlands - FAR - Hydro-Gardens - IPM Labs • Koppert - M \& R Durango - Natural Insect - Natural Pest Control • Natural Pest Controls - Nature's Alternative - Nature's Control • Peaceful Valley - Plant Sciences - Praxis - Rincon-Vitova - W. Neudorff Nephus reunioni: Amos Rubin Scymnus sp. Amos Rubin

\section{Least-Toxic Chemical Controls}

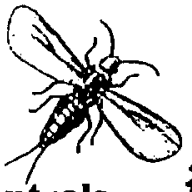

Diatomaceous earth, with pyrethrin: See ANTS

Insect Growth Regulator: Sandoz (Enstar)

Insecticidal Oil:

Highly refined oll (from fish):

IFM (Crocker's Fish Oil) - Peaceful Valley (Fish Oil)

Highly refined oil (from petroleum):

Mycogen, Gardens Alive!, Harmony, IFM, Peaceful Valley (Sunspray) • Valent USA, Harmony, Peaceful Valley (Volck Supreme Spray)

Highly refined oil (from petroleum), with sulfur:

Necessary (Dormant Oil \& Lime Sulfur)

Highly refined oil (from vegetables):

ARBICO (Spreadall, 93\% cottonseed) • Peaceful Valley (Stoller Natur'l Oil)

\section{Insecticidal Soap: See APHIDS}

Neem or azadirachtin:

AgriDyne, Necessary, Harmony (Azatin) • Grace-Sierra, Harmony

(Margosan-O) • Ringer, IFM, Harmony, Gardenville (BioNeem)

\section{MITES}

\section{Identification \& Monitoring}

Traps:

Phero Tech (Varroa Mite Trap)

\section{Physical Controls}

Furniture covers for dust mites:

Allergy Control Products - Coverplus (Ventflex)

Vacuums:

Rexair (Rainbow Water Vacuum)

\section{Biological Controls}

Microbial Insecticides:

Abamectin:

Harmony (Avid)

Predators:
Lady beetle, Stethorus punctillum (for European red spider mite): Praxis

Predatory bugs:

Orius albidipennis: Bunting • Koppert

Orius laevigatus: Koppert

Orius majusculus: Biological Crop • Bunting - Koppert • Sautter \& Stepper $\cdot$ W. Neudorff

Orius insidiosis: Bunting • Gartnerei $\bullet$ IPM Labs $\cdot$ Koppert $\cdot$ Nature's Control

Orius spp.: Biobest $\cdot$ Biolab

- Chr. Hansens - Duclos •

Natural Insect Peaceful Valley

- Plant Sciences

Orius tristicolor: ARBICO

- Bozeman • IPM Labs • Praxis

Predatory mites:

Amblyseius californicus

(for two-spotted spider mite): ARBICO

- Bio Ag • Bio-Agronomics • Biotactics

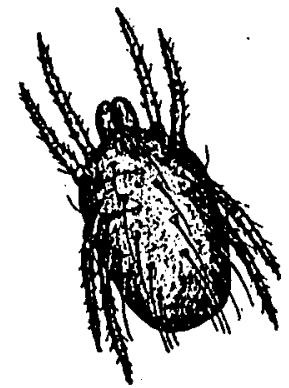

- BoBiotrol - Bozeman - Coast - FAR - Hydro-Gardens - IPM Labs -

Kuida $\cdot M$ \& R Durango - Natural Insect - Natural Pest Controls •

Nature's Control - Peaceful Valley - Rincon-Vitova • Sierra Ag • TriCal

Amblyseius cucumeris or Neoseiulus cucumeris(for two-spotted spider mite): Applied Bionomics - ARBICO - Better Yield - Biobest - Biofac • Bunting - Chr. Hansens - Gartnerei - IPM Labs - Koppert - M \& R Durango - Nature's Alternative - Natural Insect - Natural Pest Control • Natural Pest Controls - Peaceful Valley - Pest Management - Praxis • Rincon-Vitova $\cdot$ W. Neudorff

Amblyseius fallacis (for two-spotted spider mite, European red mite): Applied Bionomics

Mesoseiulus longipes or Phytoseiulus longipes (for two-spotted spider mite): ARBICO - Biotactics - Bozeman - FAR - Hydro-Gardens - IPM • M \& R Durango - Natural Insect - Nature's Control - Peaceful Valley • Praxis

Metaseiulus occidentialis or Galendromus occidentalis: ARBICO • Bio $\mathrm{Ag} \cdot$ Bio-Agronomics - Biological Control - Biological Pest - Biotactics - BoBiotrol - Bozeman - Central Coast - Coast - FAR - IPM Labs • Koppert - Kuida $-M$ \& R Durango - Natural Insect $\cdot$ Natural Pest Controls - Nature's Control - Peaceful Valley - Plant Sciences - Praxis • Rincon-Vitova $\cdot$ TriCal

Orientiseius rickeri or Anthoseius rickeri: Praxis

Phytoseiulus persimilis (for two-spotted spider mite): Applied Bionomics - ARBICO - Azienda - Barmann - Beneficial Resources • Better Yield - Bio Ag - Bio-Agronomics - Biobest - Biocontrol - Biofac • Biolab - Biological Control - Biologic al Crop - Biological Pest • Biotactics - BoBiotrol - Bozeman - Budai - Bunting - CO. DEA • Central Coast - Chr. Hansens - Coast - Duclos - ETS - English Woodlands - FAR - Gartnerci - Hawkesbury - Hydro-Gardens - IPM Labs Koppert - Kuida - M \& R Durango - Natural Insect- Natural Pest Control - Natural Pest Controls - Nature's Alternative - Nature's Control • Peaceful Valley - Pest Management - Plant Sciences - Praxis - RinconVitova - Sautter \& Stepper - Sweetbriar - TriCal - W. Neudorff - Zaklad

Typhlodromus athiasae (for red mite): Amos Rubin

Typhlodromus limonicus: Praxis

\section{Least-Toxic Chemical Controls}

Benzyl Benzoate:

Fisons (Acarosan) 
Insect Growth Regulators:

Methoprene:

Zoecon (Altosid)

Insecticidal Oil:

Highly refined öll (from fish):

IFM - Peaceful Valley

Highly refined oll (from petroleum):

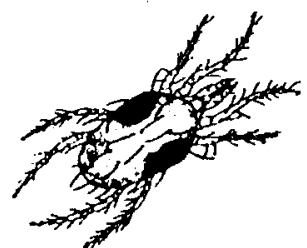

Monterey, Harmony (Saf-T-Side) - Valent USA, Harmony, Peaceful

Valley (Volck Supreme Spray) - Mycogen, Gardens Alive!, Harmony,

IFM, Peaceful Valley (Sunspray)

Highly reflned oll (from petroleum), with sulfur:

Necessary (Dormant Oil \& Lime Sulfur)

Highly reflned oll (from vegetables):

ARBICO (Spreadall, $93 \%$ cottonseed) • Peaceful Valley (Stoller Natur'1 Oil)

\section{Insecticidal Soap: See APHIDS}

Pyrethrin:

Hess \& Clark (Capsul-Ban) - Roxide - Whitmire (PT 3-6-10 Aero-Cide, PT-170 X-Clude, PT-175 MicroCare, PT-565 Plus)

Pyrethroid (nuvalinate):

Zoccon, Brushy Mountain (Apistan Strips)

Pyrethrold (permethrin-soaked cotton balls given to laboratory

rodents to kill ectoparasite vectors):

Ecohealth (Mite Arrest)

Repellent Garlic Spray (for spider mite):

Guardian Spray, New Earth (ENVIRepel, Guardian Spray) • Garlic

Research Labs, Agape (Garlic Barrier)

Silica Aerogel: See ANTS

Tannic acid:'

Allergy Control Products

\section{MOSQUITOES}

\section{Identification \& Monitoring}

Mosquito larvae collection devices:

Clark Mosquito Control (Dippers)

\section{Physical Controls}

Screening:

BioQuip • REI

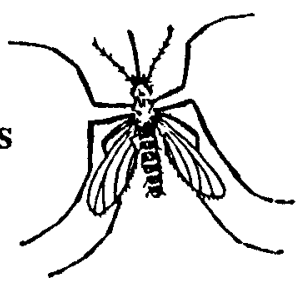

\section{Biological Controls}

Microbial Insecticides:

Bacillus thuringiensis israelensis (BTI) for mosquito \& blackfly larvae:

Abbott Laboratories (Vectobac) - Novo Nordisk, Rol-Eko, Peaceful

Valley (Skeetal, Bactimos) - Sandoz Crop Protection (Teknar) - Summit,

Nitron,SSutton, Smith \& Hawken, Natural Insect, Gardenville (Mosquito

Dunks) - Bozeman, Peaceful Valley, Gardenville, Necessary (Mosquito

Control) - Summit, Rol-Eko, Peaceful Valley, Novo Nordisk (Bactimos)

Parasitoids:

Encarsia opulensa (for citrus blackfly): Praxis

Predators:

Mosquito fish, Gambusia affinis: J. Reilly (Fish shipped May-July) •
Natural Pest Controls $\cdot$ Sea Ranch

\section{Least-Toxic Chemical Controls}

Insecticidal Oil: See APHIDS

Insect Growth Regulators:

Methoprene pellets, Zoecon (Altosid)

Pyrethrin:

Waterbury (Purge III)

Repellents:

Bozeman, Gardenville, Natural Insect(Skeeter Shooo Botanical)

DEET repellent:

REI (Cutter's, Jungle Juice)

\section{MOTHS (See Caterpillars)}

\section{NEMATODES}

\section{Biological Controls}

Entomopathogenic fungus:

International Mycological Institute

Natural chitin-protein nematicide:

Igene Biotechnology, IFM, Harmony, Necessary; Peaceful Valley, Gardenville (ClandoSan)

\section{SAWFLIES}

\section{Physical Controls}

Traps and Sticky Tape:

Phero Tech (Chroma)

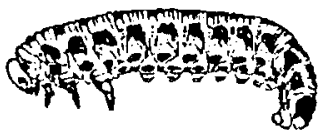

\section{Least-Toxic Chemical Controlś}

\section{Insecticidal Soap: See APHIDS}

Neem or azadirachtin:

AgriDyne, Necessary, Harmony (Azatin) - Ringer, IFM, Harmony, Gardenville (BioNeem) • Grace-Sierra, Harmony (Margosan-O)

\section{SCALES}

\section{Identification \& Monitoring}

Pheromones \& Traps:

ARBICO, Harmony (Catch-em) - Hercon, Great Lakes, Pest Management (Multi-Pher, Unitrap. Delta Traps) - Scentry, Pest Management, Great Lakes, Arbico, Bozeman (Scentry Gypsy Moth) - Scentry, Great Lakes, Arbico, Bozeman, Pest Management, Natural Insect (Delta \& Wing Trap)

Sticky Trap/Tape:

Arbico - Bozeman - Great Lakes • Pest Management - Scentry 


\section{Physical Controls}

Barriers \& Exclusion Devices:

Bozeman - Gardens Alive!

Screening:

Hydro-Gardens (Fly Barr)

Sticky Adhesive:

Seabright, Great Lakes, Harmony, Phero Tech (Stickem Special)

Traps and Sticky Tape:

Bozeman - Gardens Alivel • Harmony - IFM - IPM Labs - Phero Tech (Chroma) - Necessary

\section{Biological Controls}

\section{Parasitoids:}

Aphytis lingnanensis (for California red scale): Amos Rubin - Bugs for Bugs

Aphytis melinus (for red scale parasitoid): ARBICO - Bassett's - Bio Ag

- Bio-Agronomics - Biological Pest - BoBiotrol - Coast - FAR - IPM

Labs - Kuida - Natural Insect - Natural Pest Controls - Peaceful Valley •

Praxis - Rincon-Vitova - Sespe Creek - Sierra Ag - TriCal

Coccophagus lycimnia: Praxis

Comperiella bifasciala (for red scale): Praxis

Metaphycus helvolus (for black scale): ARBICO • FAR - IPM Labs •

Natural Insect - Natural Pest Controls - Peaceful Valley - Praxis •

Rincon-Vitova $\cdot$ Sespe Creek

Microterys flavus (for brown scale): ARBICO - Natural Pest Controls • Praxis

Parasitic mite, Cunaxoides spp.: Praxis

Predators:

Lady beetles:

Chilocorus baileyii (for armored scale): Bugs for Bugs

Chilocorus circumdatus (for citrus snow scale, armored scale): Bugs for Bugs

Chilocorus spp.: Praxis

Cybocephalus sp.: Praxis

Lindorus lophanthae: ARBICO - Bozeman - Bugs for Bugs - Canadian Insectaries - IPM Labs - Natural Insect - Praxis - Rincon-Vitova

Predatory mite, Euseius spp. (for scale general, sweetpotato whitefly): Praxis

\section{Least-Toxic Chemical Controls}

Insect Growth Regulator:

Sandoz (Enstar)

Insecticidal Oil: See APHIDS

Insecticidal Soap: See APHIDS

Pyrethrin:

Johnny's, Peaceful Valley (Pyrenone)

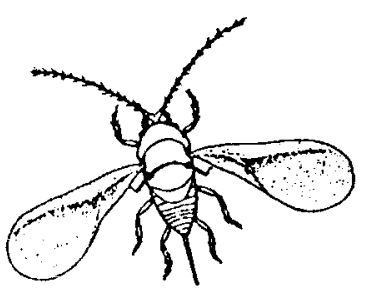

\section{SILVERFISH}

\section{Least-Toxic Chemical Controls}

Borate-Based Insecticides:

Waterbury (Mop-Up)- Whitmire (PT 240 or PT 249 Perma-Dust) •
Peaceful Valley (Roach Powder)

Diatomaceous Earth: See ANTS

Pyrethrin:

Hess \& Clark (Capsul-Ban) • Roxide • Whitmire (PT 3-6-10 Acro-Cide, PT-170 X-Clude liquid concentrate, PT-175 MicroCare, PT-565 Plus)

Silica Aerogel: See ANTS

\section{SLUGS and SNAILS}

\section{Physical Controls}

Barriers \& Exclusion Devices:

Copper Snall barrier:

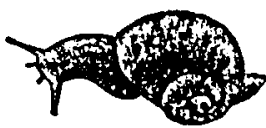

Custom Copper, Harmony, The Natural Gardening, Gardenville,

Peaceful Valley (Snail-Barr)

\section{Traps:}

Gardenville - Sound Merchandise (Garden Sentry) •

Nitron, Peaceful Valley, Gardens Alive!, ARBICO (Slug Saloon)

Trap with barley/rice yeast bait:

Gardener's Supply (Slug Trap)

\section{Biological Controls}

Predators:

Predatory snail, Rumina decollala (for brown garden snail): ARBICO .

C. H. Musgrove • FAR - Peaceful Valley • Rincon-Vitova - Sespe Crcek Predatory fly, Tetanocera spp. (for European slug): Praxis

\section{Least-Toxic Chemical Controls}

Insecticidal Soap: See APHIDS

\section{TERMITES}

\section{Identification \& Monitoring}

Odor Detector:

Termitect II (Odor Detector)

Rigid borescope for

detecting termites:

Fibertron, Van Waters \& Rogers (Econoscope)

Trained Dogs:

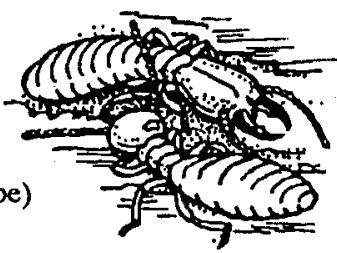

Beacon Dogs • Clark Pest • Industrial Narcotic • TADD

\section{Physical Controls}

Electrical Treatment (for drywood termite \& boring beetles):

ETEX (Electrogun)

Heat Treatment:

Isothermics (Licenses companies - Call for Local Practitioners) •

Mission Pest Control • Steffenson (for Northern Califomia only)

Microwave Treatment:

The Termite Inspector 
Sand Barrier:

Live Oak Structural

\section{Least-Toxic Chemical Controls}

Borate Termiticide \& Wood Preservative:

NISUS (Bora-Care) • NISUS, CSI (Impel) • U.S. Borax (TimBor)

Pyrethróld Soll Treatment:

Zeneca (Demon TC with cypermethrin, Torpedo with permethrin)

Silica Aerogel (In wall volds and attics to repel

drywood termites): See ANTS.

\section{THRIPS}

\section{Identification \& Monitoring}

Pheromones \& Traps:

AgriSense (Magnet) - Great Lakes, Phero Tech (Unitrap) • Phero Tech (Phero Tech Lures) - Trécé, Gardens Alive!, Great Lakes, Olson, Pest Management (Pherocon)

Sticky Trap/Tape:

Arbico • Bozeman • Great Lakes• Pest Management • Scentry

\section{Physical Controls}

Screening:

ATI (Greenhouse screen) • Tie-Net

Traps and Sticky Tape:

Gardenville • Hansens Bio • Harmony • Natural Insect • Olson • Pest

Management $\cdot$ Phero Tech

\section{Biological Controls}

Parasitoids:

Thripobius semiluteus: ARBICO • FAR •

Natural Insect • Praxis • Peaceful Valley

Predators:

Predatory bugs:

Deraeocoris brevis: Applied Bionomics

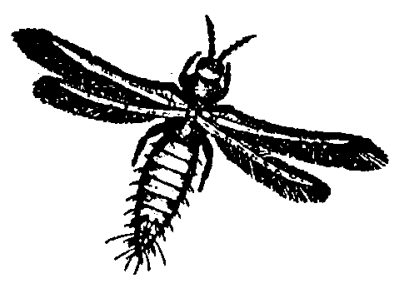

Orius albidipennis: Bunting $\cdot$ Koppert

Orius insidiosis: Beneficial Resources - Bunting • Koppert • IPM Labs • Gartnerei - Nature's Control

Orius laevigatus (for western flower thrips): Amos Rubin - Koppert

Orius majusculus: Biological Crop • Bunting • Koppert - Sautter \& Stepper $\cdot$ W. Neudorff

Orius spp.: Biobest - Biolab • Chr. Hansens - Duclos - Natural Insect • Peaceful Valley $\cdot$ Plant Sciences $\cdot$ Rincon-Vitova

Orius tristicolor: ARBICO $\cdot$ Bozeman • IPM Labs • Praxis

Predatory mites:

Amblyseius barkeri: FAR - IPM Labs • M \& R Durango - Nature's

Control • Natural Insect • Sautter \& Stepper • W. Neudorff

Amblyseius cucumeris or Neoseiulus cucumeris: ARBICO - Applied Bionomics - Better Yield • Biobest - Biofac $\cdot$ Bozeman - Bunting $\bullet$ Chr. Hansens • Gartnerei - IPM Labs - Koppert • M \& R Durango - Nature's Altemative - Natural Insect - Natural Pest Control - Natural Pest Controls • Nature's Control • Peaceful Valley • Pest Management • Plant
Sciences $•$ Praxis $・$ Rincon-Vitova $\cdot$ W. Neudorff

Amblyseius mckenziei: Hydro-Gardens • Natural Insect

Amblyseius spp.: Beneficial Resources • Biological Crop

Hypoaspis miles: Applied Bionomics • IPM Labs • Natural Insect

\section{Least-Toxic Chemical Controls}

\section{Diatomaceous Earth: See ANTS}

\section{Insecticidal Soap: See APHIDS}

Neem or azadirachtin:

AgriDyne, Necessary, Harmony (Azatin) - Ringer, IFM, Harmony, Gardenville (BioNeem) • Grace-Sierra, Harmony (Margosan-O)

Pyrethrin:

Johnny's, Peaceful Valley (Pyrenone) • Roussel, Insects Limited (Kicker)

\section{Repellent Garlic Spray:}

Garlic Research Labs, Agape (Garlic Barrier) - Guardian Spray, New Earth (ENVIRepel, Guardian Spray)

Ryania (restricted for use on commmercial products): Agri-Systems • Gardens Alive! • Harmony • IFM • Necessary • Peaceful Valley

\section{TICKS}

\section{Physical Controls}

Curved tweezers:

BioQuip Products (foreceps)

Tweezers, magnifier \& specimen storage bag: REI (Tick Solution Kit)

\section{Least-Toxic Chemical Controls}

Dlatomaceous Earth, with pyrethrin:

Organic Plus - Permaguard, Pristine, Nitron (Permaguard Pet \& Animal)

- Pristine, Nitron, Gardenville (Permaguard Houschold)

Insecticidal Oil:

Cltrus oil (d-limonene) extract:

Hansen's Pet Systems (Citrus Plus

Shampoo, Spray) • Vet Express

(VIP Pet Spray, Shampoo, Dip)

Insecticidal Soap:

With citrus ofl:

Ringer, Bozeman, Gardens Alive!,

Harmony, IFM, Nitron, Johnny's,

Smith \& Hawken, Peaceful Valley,

Charley's, Gardenville (Safer Insecticidal Soap)

With citrus oil \& pyrethrum:

Harmony, Bozeman, Gardens Alive!, Pest Management Supply (Flea \& Tick Attack)

With pyrethrum:

Ringer, Bozeman, Gardens Alive!, Harmony (Flea \& Tick Attack) • Ringer, Gardens Alive!, Harmony, Nitron, Gardenville (Safer Yard and Garden)

Pyrethrin:

Del (Lice, Tick \& Flea Killing Spray) 
Pyrethroid (permethrin-soaked cotton balls taken to rodent nests to kill ectoparasite vectors):

Ecohealth, Harmony, Peaceful Valley (Damminix)

Repellents:

DEET repellent:

REI (Cutter's, Jungle Juice)

Permethrin repellent:

Forestry Suppliers (Permanone) • Roussel (Permanone)

Silica Aerogel: see ANTS

\section{WASPS (See Yellowjackets)}

\section{WEEVILS (See Beetles)}

\section{WHITEFLIES}

\section{Identificảtion \& Monitoring}

Pheromones \& Traps:

AgriSense (Magnet) - Trécé, Gardens Alive!, Great Lakes, Olson, Pest Management (Pherocon)

Sticky Trap/Tape:

Scentry, Great Lakes, Arbico, Bozeman, Pest Management (Versagard)

\section{Physical Controls}

Screening:

ATI (Greenhouse screen)• Hydro-Gardens (Fly Barr) • Tie-Net

Sticky Adhesive:

Tanglefoot, Arbico, Bozeman, Great Lakes, Pest Management,

Gardenville, Harmony, Natural Insect Control, Charley's (Tangle-Trap)

Traps:

Ringer, Gardenville (Safer Flying Insect)

Sticky trap/tape:

Arbico $\cdot$ Gardenville $\cdot$ Hansens Bio •

Natural Insect - Phero Tech (Chroma) -

Olson, Great Lakes, Pest Management

(Stiky Stuff, Stiky Traps) • Seabright (Sticky Traps)

\section{Biological Controls}

Microbial Insecticides:

Verticillium lecanii fungus (for greenhouse use): Koppent (Mycotal, Vertalec)

Parasitoids:

Encarsia formosa (for greenhouse whitefly): ARBICO - Applied

Bionomics - Barmann - Beneficial Resources - Better Yield - Biobest •

Biofac - Biolab - Biological Crop - Bozeman - Budai • Bunting • CIE •

CO. DEA - CTIF - Chr. Hansens - Duclos - English Woodlands - FAR • Gartnerei - Hydro-Gardens - IPM Labs - Koppert - M \& R Durango • Natural Insect - Natural Pest Control - Natural Pest Controls • Nature's Alternative - Nature's Control - Peaceful Valley - Pest Management • Plant Sciences • Praxis $\bullet$ Rincon-Vitova $\cdot$ Sautter \& Stepper $\cdot$ W. Neudorff $\cdot$ Zaklad
Eretmocerus californicus (for sweetpotato whitefly): IPM Labs • Praxis

Predators:

Lady beetle, Delphastus pusillus (for sweetpotato whitefly): Amos Rubin - Applied Bionomics - ARBICO - Bozeman - Biological Control • Hydro-Gardens • IPM Labs - Natural Insect • Pest Management - Praxis

- Rincon-Vitova

Predatory bug, Macrolophus caliginosus: Beneficial Resources $•$ Biolab

Predatory mite, Euseius rubini: Amos Rubin

Predatory mite, Euseius spp.: Praxis

\section{Least-Toxic Chemical Controls}

\section{Diatomaceous Earth: See ANTS}

Insect Growth Regulators:

Sandoz Crop Protection (Enstar)

Insecticidal Oil:

Highly refined ofl (from petroleum):

Monterey, Harmony (Saf-T-Side) - Mycogen,

Gardens Alive!, Harmony, IFM, Peaceful Valley (Sunspray)

Highly refined oil (from vegetables):

Peaceful Valley (Stoller Natur'l Oil)

Insecticidal Soap: See APHIDS

Neem or azadirachtin:

AgriDyne, Necessary, Harmony (Azatin) - Ringer, IFM, Harmony, Gardenville (BioNeem) - Grace-Sierra, Harmony (Margosan-O)

Pyrethrin:

Johnny's, Peaceful Valley (Pyrenone) • Roussel, Insects Limited (Kicker)

Pyrethrum:

Monterey (Bug-Buster-O)

Repellent Garlic Spray:

Guardian Spray, New Earth (ENVIRepel, Guardian Spray) • Garlic

Research Labs, Agape (Garlic Barrier)

\section{WORMS (See Caterpillars)}

\section{YELLOWJACKETS}

\section{Identification \& Monitoring}

Pheromones \& Traps:

AgriSense, Great Lakes, Pest Management (Trappit) • Consep, IFM, Harmony, Pest Management (Checkmate) • Consep, Smith \& Hawken (Consep Yellowjacket Trap) - Phero Tech (Phero Tech Lures) • Phero Tech, Great Lakes (Unitrap) • Trécé, Gardens Alive!, Great Lakes, Olson, Pest Management (Pherocon)

\section{Physical Controls}

Beekeeping equipment for use on bee swarms, yellowjacket nest removal:

A.I. Root (bee veil, gloves, suits) • Dadant \& Sons (Bee veils \& coveralls) 
Bee swarm traps:

Pest Management, Great Lakes, Arbico, Bozeman (Swarm Trap)

Traps:

Baley's (Pyramid) - Seabright, Great Lakes, Harmony, Phero Tech, Pest Management (Yellowjacket Inn) - Sterling (Rescuel Yellowjacket) • Woodstream, Brody, Great Lakes, Peaceful Valley, Natural Insect (Victor Fly-In-Saucer)

\section{Least-Toxic Chemical Controls}

Boric acid 20\% aerosol:

Whitmire (PT 240 or PT 249 Perma-Dust)

Diatomaceous Earth (with pyrethrin): See ANTS

Insecticidal Soap:

With citrus oll \& pyrethrum:

Ringer, Peaceful Valley (Wasp \& Hornet Attack)

Pyrethrin:

Hess \& Clark (Capsul-Ban) - Roxide - Whitmire (PT 3-6-10 Aero-Cide, PT-565 Plus)

With components that "freeze" wasps:

Mine Safety Appliance (Wasp-Stopper DOA)

Resmethrin plus components that "freeze" wasps:

Roxide (Revenge Wasp \& Hornet Killer)

Silica Aerogel (with pyrethrin): See ANTS

Wasp/Bee Sting Medication:

Self-injected epinephrine:

Center (EpiPen) • Miles (Ana-Guard) • Spencer-Mead 3

\section{MISCELLANEOUS}

\section{Educational Materials}

Databases (Pest Management):

Bio-Integral Resource Center - California Statewide IPM Project

(IMPACT Database)

On-line, CD-ROM database of registered pesticide products,

chemical fact sheets, MSDS sheets:

NPIRS User Services (NPIRS)

Pest Management Publications \& AV Products:

Books on agriculture, hortlculture, pest management: agAccess

Integrated pest management publications, slides \& videos:

Bio-Integral Resource Center (BIRC)

Slides on blological control:

$\mathrm{BCW}$

Slides, reference library, trainlng manuals:

Van Waters \& Rogers

Trainlng kit for pestlcide applicators:

Abraxas Communications Ltd. (video training package)

Vldeos introducing IPM In agriculture, landscaplng, \& greenhouses, two pest ID slides kits:

San Luis Video

\section{Identification \& Monitoring}

Computers \& Software:

Apple scab forecasting equipment:
Pest Management Supply (PSAOC consultant software)

Portable data recording equipment:

Omnidata (Biophenometers)

Information/Identification Services \& Supplies:

Entomological specimens $(10,000$ specles): Combined Scientific

Supplies, Great Lakes

Equipment, supplles \& books: BioQuip - Pest Management

Ichneumonid identification service: American Entomological Institute

Identification and Information service for pests and beneficlal insects: International Institute of Entomology

Identification of insect pathogens: Consulting Diagnostic Service

Information, identification of agricultural, urban insects: Cooperative Extension Service (local of fices)

Information service on blological control: International Institute of Biological Control

Monitoring Devices:

Electronic insect counter: Automata (Data-Lynx BUGCOUNT)

Flashlight with red filters: Streamlight, Brody (Streamlight)

Inspectlon mirror: Applied Bionomics, Harmony Farm (Magnifier hand lenses, headband magnifiers) - Brody

Microscopes: Pest Management (Donegan Optical) - RF Inter-Science, Pest Management (Macroscope 25, Macroscope 45, Macroscope 18-36 Zoom)

Moisture meters: Delmhorst, Brody (Delmhorst)

Sweep nets: Oakfield Apparatus

Temperature monitor: Great Lakes (Taylor Min/Max Thermometer)

- IFM (CropClok 1000 Temperature Integrator, Dickenson Self-

Contained Temperature Recorders)

Vacuums: ARBICO (Bug Sweep) • BioQuip Products (Insect Vacs) • D.

VAC Company (D-VAC) • McCluney • Gardener's Supply (Bug Vacuum)

Weather monitor \& disease forecasting system: Automata (Data-Lynx Degree Day) $\cdot$ Ncogen (EnviroCaster) $\cdot$ Pest Management (Pessl Metos Products)

Pesticide Residue Detection:

Aquatic sampling equipment: Wildlife Supply

Detection of carbamate, organophosphàte $\&$ thlophosphate residues In air, water, soll or surfaces: Great Lakes, IFM (Enzytec) - Neogen (Ver atox) • Quantix (For atrazine, triazine, alachlor, metolachlor, benomyl, chlorothalonil, trifluralin, imazaquin, PAH's)

\section{Physical Controls}

Row Covers:

Charley's (Harvest-Gard)

\section{Biological Controls and Supplies}

Artificial Diets:

For lacewings \& lady beetles:

Rincon-Vitova - The Ladybug Company

Wheast for lacewings $\&$ lady beetles:

Bozeman - CRS Company • Peaceful Valley, Charley's (Pred-Feed) • Natural Insect

Fluon Insect Barrier:

Zeneca 


\section{Habitat:}

Bat house to encourage insect predation by bats:

Gardener's Supply (Bat House)

Seed mixture for beneficial insect habitat:

Gardens Alive! (Haven Flowering Herbs) - IFM (Bug \& Breakfast

Blend) - Peaceful Valley (Good Bug Blend, Border Patrol)

Transplants for beneficial insect habitat:

Comflower Farms

Insect and Microbial Rearing Supplies:

Autolysed yeast (rearing medium, fruitfly attractant, balting control): Bugs for Bugs

Sitotroga cerealella (moth eggs for rearing medium): Kunafin

Supplies for rearing insect pathogens:

International Mycological Institute - GIBCO

(for rearing insect pathogens) • Oliver

Pollination aids:

Scentry (Bee-Scent)

Pollinators: bumble bee;

Bombus spp.: W. Neudorff

Pollinators: house flies:

Rincon-Vitova

\section{Predators:}

Chinese praying mantid,

Tenodera aridifolia sinensis:

ARBICO - Bozeman -

Gardenville $\cdot M$ \& R Durango

- Natural Insect - Natural Pest Controls

- Peaceful Valley - Praxis - Smith \& Hawken

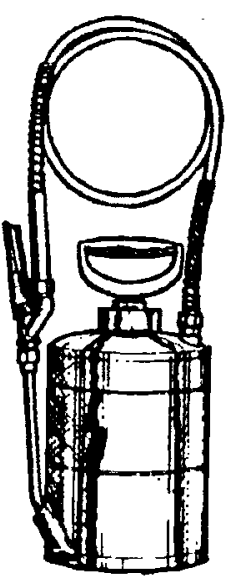

- Unique Insect Control

Red hybrid earthworms, Lumbricas sp.:

Unique Insect Control

\section{Pesticide Application Equipment}

\section{Application Equipment:}

B\&G Equipment (B\&G Dusters) - Roussel UCLAF (Actisol Duster) • Technicide (Technicide Duster) • Van Waters \& Rogers (Getz Gun) • Pristine (Murayama Back Pack) • Bozeman (Dustin Mizer) • IFM • Nitron, Peaceful Valley, Garden's Alive!, Charley's, Gardenville (Dustin Mizer) • Parker (P.E.S.T. Power Applicator) • Woodstream, Peaceful Valley (Pest Pistol)

Application equipment for structural pest control:

B \& G $\cdot$ Harmony $\cdot$ Necessary Trading $\cdot$ Peaceful Valley

Bait applicator:

Specialty Products (BaitGun)

Electrostatic sprayers:

Electrostatic Spraying Systems

Sprayers:

Bozeman • Gardener's Supply (Spraydoc) • Gardenville (Berthoud sprayer)

- Green Pro • Harmony • IFM (Aeropure Sprayer) - Necessary Trading

Respirators:

BWS - Harmony

- Lab Safety Supply

(full hood respirator) - Necessary Trading

- Survivair (Series 86 Disposable APR)

(Call Survivair for local distributors)

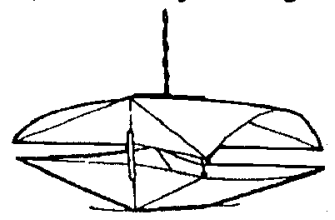

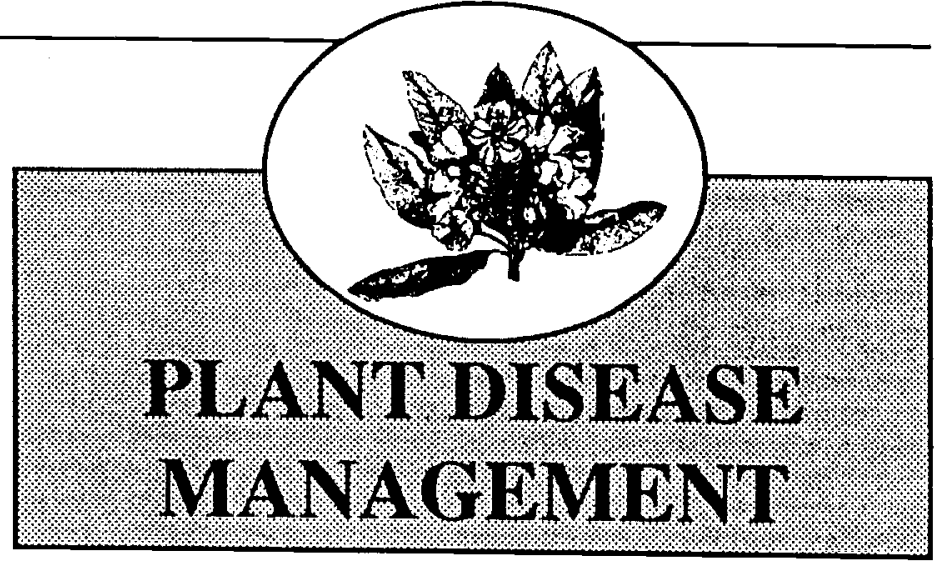

\section{CROWN GALL DISEASE}

\section{Biological Controls}

Microbial Fungicides:

Agrobacterium radiobacter strain K84: AgBioChem, Necessary, Harmony (Galltrol-A, Gallex) - New BioProducts, IPM Labs (NORBAC 84-C)

\section{FUNGAL FOLIAGE/FLOWER FRUIT PATHOGENS}

\section{Identification \& Monitoring}

Apple scab forecasting equipment:

Harmony (Qualimetrics Hygrothermograph) • Pest Management (VENTEM prediction software)

Weather monitor $\boldsymbol{\&}$ disease forecasting system (for fire blight):

Pest Management (MAR YBLYT)

\section{Physical Controls}

Fungal Barriers:

Antitranspirants:

Aquatrols (FoliCote) - Green Pro (Vita Coat) - Mine Safety, Miller (Vapor Gard) • Mycogen (ForEverGreen) • PBI/Gordon (Transfilm) • Precision (Preserve) - Wilt-Pruf (Wilt-Pruf) - Nature's Touch (Leaf Cote Clear) - Gardenville (Cloud Cover)

Habitat:

Styrofoam blocks to increase ventilation:

Beaver Plastics, First Choice (Ventblocks)

\section{Horticultural Controls}

Disease-Resistant Roses:

Heritage $\cdot$ High Country $\cdot$ Hortica

\section{Biological Controls}

Microbial Fungicides:

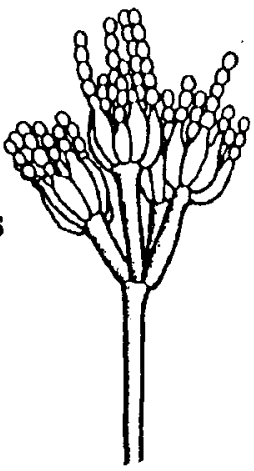

Glomus intraradix (for pythium, fusarium): Premier (Mycori-Mix, potting soil innoculated with fungus) 
Phlebia gigantea: Kemira (Rotstop)

Streptomyces griseoviridis strain K61 (for fusarium, damping off. pythium, phytophthora, etc.): Kemira, Hydro-Gardens, Ag Bio Development (Mycostop)

Trichoderma sp.: Binab (Binab-T), to treat tree wounds

Seed Treatment:

Bacillus subtilis: Gustafson (Kodiac)

Pseudomonas cepacia: Stine Seed (Blue Circle)

Pseudomonas fluorescens: Ecogen (Dagger)

\section{Least-Toxic Chemical Controls}

\section{Copper \& Sulfur Fungicides:}

Monterey (Nordox, Copper Count N, Micronized Sulfur) • Peaceful Valley, Gardenville (Kocide, Bordeaux) • Harmony • Gardenville.

Necessary (Top Cop. Top Cop with sulfur, That Liquid Sulfur)

Flowable sulfur formulation:

Ringer, Harmony, Charley's, IFM, Gardenville (Safer Garden Fungicide)

Highly refined oll (from petroleum) \& sulfur:

Necessary

\section{Microbial Fungicides:}

Streptomycin antiblotic (for pear, apple fire blight):

Necessary • Peaceful Valley (Agrimycin)

Terramycin antiblotic (for pear fire blight, peach bacterial spot): Peaceful Valley (Mycoshield)

\section{TREE WOUND PATHOGENS}

\section{Physical Controls}

Herbal tree wound seal:

Pest Management (Tanglefoot Tree Paint)

\section{TURF DISEASES}

\section{Physical Controls}

Turf Aerator:

The Natural Gardening (Gorilla Core Aerator)

\section{Biological Controls}

Microbial Soil Amendments:

New Era Farm Service

(composted soil ammendments)

Beneficial microorganisms that attack turf pathogens: Agro-Chem (Gteen Magic) • Unique Products (Wonderlawn, Mega Flowers \& Vegetables, Bioturf, Natural C) $\cdot$ Ringer, Peaceful Valley (Restore) - Nature's Touch • (Nutra Aid, Strengthen \& Renew)

\section{MISCELLANEOUS}

\section{Educational Materials}

Pest Management Publications \& AV Products:

Integrated pest management publications, slides $\&$ videos:

Bio-Integral Resource Center (BIRC)

\section{Identification \& Monitoring}

Apple scab forecasting equipment:

Great Lakes IPM (Belfort Leaf Wetness Recorder)

Information/Identification Services:

Fungal identlfication service: Intemational Mycological Institute Information, identification of agrlcultural, urban insects: Cooperative Extension Service (local offices)

Information service on biological control of microbes: International Institule of Biological Control

Nematode identification service: C.A.B. International Institute of Parasitology

Plant Disease Detection Kits (for fusarium mold, pythium, phytophthora, rhizoctonia, sclerotina, brown patch, dollar spot): Neogen Corp. (Alert, Agri-Screen, Reveal)

Plant Disease Detector: Automata Inc. (Leaf Moisture Sensor)

Plant Disease Prediction Software (for general fungal disease, apple scab, botrytis, powdery mildew): Automata Inc. (GET WELL)

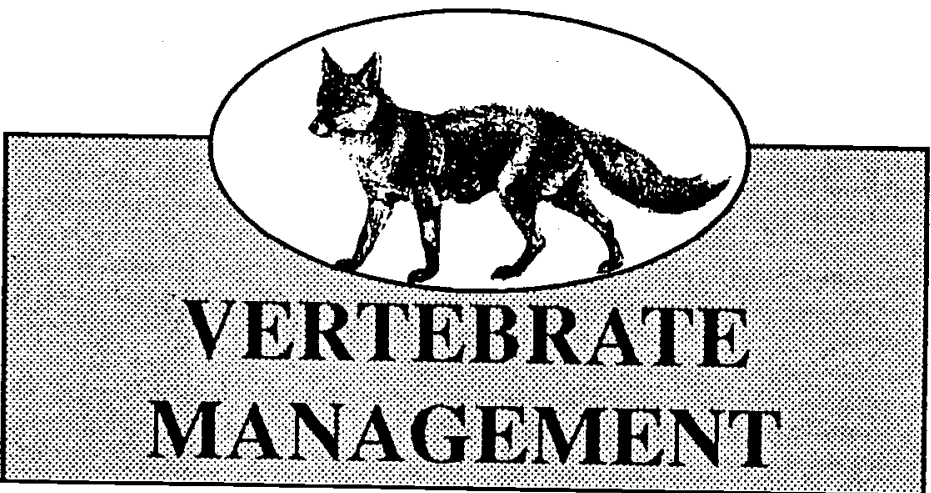

\section{BATS}

\section{Educational Materials}

Bat House Construction Booklet:

Bat Conservation International, Plow \& Hearth, Peaceful Valley (Bat house)

\section{Identification \& Monitoring}

Flashlight with red filters:

Streamlight, Brody (Streamlight)

Physical Controls

Bat proofing service using excluders:

Bay Area Bat Protection

Copper mesh hole filler: 
Allen Special Products (Stuf-Fit)

\section{Netting:}

Wildlife Control (Bat Kit, Swallow \& Woodpecker Kit for home-made bat check valve, Wildlife Net) - Pest Management (Wildlife Net)

\section{BIRDS}

\section{Identification \& Monitoring}

\section{Landing nets:}

Nichols

\section{Physical Controls}

Barriers \& Exclusion Devices:

The Huge Company • Wildlife Control (Pigeon Barrier)

Copper mesh hole filler:

Allen Special Products (Stuf-Fit)

Metal projectors:

Cat Claw (Cat Claw)

Netting:

Wildlife Control, Pest Management (Wildlife Net) • Bird-X •

Gardenville - JT Eaton - InterNet, Bluebird, Great Lakes (Internet) •

Animal Repellents $\cdot$ Peaceful Valley $\cdot$ Nichols $•$ Nixalite (Netting) •

Sterling Marine $\cdot$ Tenax $(\mathrm{Omex}) \cdot$ Tie-Net

Wire exclusion systems:

King \& Kienlen - Shelley

Deterrents:

Alarms \& detonators:

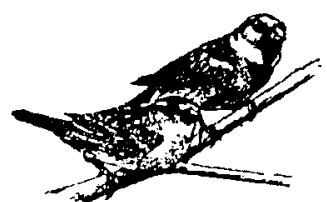

Bird-X (BirdGard, Transonic IX-L, Ultrason ET) - Brody (AV-Alarms) •

Reed-Joseph (Electronic Scare Away, Scare Away Pyrotechnics, Multi

Bang Automatic Cannon, Rotomat Revolving Platform) - Wildlife

Control (ZON Propane Cannons, Shell Crackers, Bird Bombs, Bird

Whistlers, ZON Automatic Scarecrow) - Agricultural Supply, Margo

(Zink Bird Banger) - Critter Control - Bird-X, Brody (Nixalite)

Inflating, noise-making scarecrow:

Robert Royal (Scarey Man Fall Guy)

Light deterrent:

Bird-X (Bird Strobe Light) • Brody (Bird X Lite Rays)

Reflectlve tape:

Reed-Joseph, W. Atlee (Scare-A way Reflecting Tape) - Wildlife Control, Agricultural Supply, Peaceful Valley (Mylar Silver Strips) - Bird-X (IrriTape) • Brody (Scare Hawk) • Smith \& Hawken (Birds Beware)

\section{Live Traps:}

National Live Trap • Nature House • Reed-Joseph (Trap Away) •

Tomahawk, Brody, Plow \& Hearth (Tomahawk) - Woodstream, Brody,

Great Lakes, Harmony, Peaceful Valley, Gardener's Supply, Gardenville

(Havahart, Victor) - Brody (Starling Trap)

\section{Repellents:}

Bird-X (Bird-Prof) • Brody (Roost No More)

Repelling balloons:

Reed-Joseph (Evil Eye) - ARBICO (Bird Scare Eye Balloon, Owl

Scarecrow) • Plow \& Hearth (Scare Eye) • Raven (Mylar Balloons \& Blimps) - Bird-X, Peaceful Valley, Gardenville, Pest Management (Scare Eye) - W. Atlee (Birds-Away Inflating Snake, Ollie-Owl) • Bird$\mathrm{X}$ (Terror Eyes)
Repelling kites:

Sutton $\cdot$ Cochranes

Sticky repellent:

Tanglefoot (Tanglefoot Bird Repellent)

Sticky Adhesive:

Olson, Hydro-Gardens (Stiky Stuff)

- Seabright (Stickem Special)

- The Huge Company - Brody

(Eaton's 4 the Birds Gel)

- Pest Management, Great Lakes,

Necessary, Nixalite (Tanglefoot)

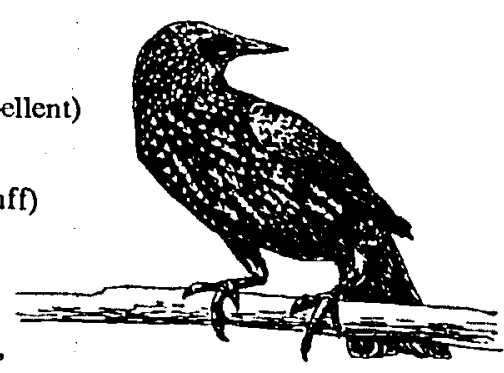

\section{Least-Toxic Chemical Controls}

Repellents:

Nixalite, Peaceful Valley, Plow \& Hearth (Ropel) - Bird-X (Bird Proof)

- JT Eaton (Birds Repellent)

\section{CATS/DOGS}

\section{Physical Controls}

Electric Fencing:

Gardener's Supply

\section{Least-Toxic Chemical Controls}

Repellents:

Sterling (Rescue! Dog \& Cat Repellent) - Peaceful Valley (Ropel) • Gardener's Supply (Champon's)

\section{DEER}

\section{Physical Controls}

\section{Deterrents:}

Alarms \& detonators:

Agricultural Supply $\cdot$ Critter Control $•$ Margo

Roadside highway reflectors to deter deer:

Strieter (Strieter-Lite)

Electric fencing:

North Bradford (Techfence) • Gardener's Supply

Tree Wrap:

Pest Management (T\&G Plastic Tree Shields) • Peaceful Valley (Spiral Tree Guard)

\section{Least-Toxic Chemical Controls}

\section{Repellents:}

Intagra, Gardener's Supply, Gardens Alive!, Harmony, Plow \& Hearth, Peaceful V alley (Deer-Away) - Harmony (National Deer Repellent) • Necessary, Gardenville, Gardens Alive!, Harmony , Peaceful Valley (Hinder Deer \& Rabbit Repellent) • Peaceful Valley, Harmony (National Scent, Miller's Hot Sauce) - Peaceful Valley (Ropel) - Gardener's Supply (Champon's) • Lakeshore (Green Screen) 


\section{LIVESTOCK PREDATORS}

\section{Physical Controls}

\section{Alarms \& Detonators:}

Reed-Joseph (Scare Away Pyrotechnics, Multi Bang Automatic Cannon, Rotomat Revolving Platform) - Agricultural Supply • Critter Control - Margo

\section{Electric Fencing:}

Gallagher, Poultry Health Services (Gallagher) - Waterford (ShockTactics, Flexinet) - Lifetime Fence - Gardener's Supply

\section{Live Traps:}

Reed-Joseph (Trap Away) - Tomahawk, Brody, Plow \& Hearth (Tomahawk) - Woodstream; Brody, Great Lakes, Harmony, Peaceful Valley, Gardener's Supply, Gardenville (Havahart, Victor)

\section{Least-Toxic Chemical Controls}

Repellents:

Peaceful Valley, Nixalite, Peaceful Valley, Plow \& Hearth (Ropel)

\section{RABBITS}

\section{Physical Controls}

Alarms \& Detonators:

Bird-X (BirdGard, Transonic IX-L, Ultrason ET)

Electric Fencing:

Gallagher, Poultry Health Services (Gallagher) • Waterford

(ShockTactics, Flexinet) • Lifetime Fence - Gardener's Supply

Netting:

Wildlife Control, Pest Management (Wildlife Net)

\section{Tree Wrap:}

Pest Management (T\&G Plastic Tree Shields) • Peaceful Valley (Spiral Tree Guard)

\section{Least-Toxic Chemical Controls}

Repellents:

Necessary, Gardens Alive!, Gardenville, Harmony (Hinder Deer \& Rabbit Repellent) - Harmony (National Scent, Miller's Hot Sauce) • Peaceful Valley (Ropcl) • Lakeshore (Green Screen) • Gardener's Supply (Champon's)

\section{RACCOONS}

Alarms \& Detonators:

\section{Physical Controls}

Bird-X (BirdGard, Transonic IX-L, Ultrason ET)

Barriers \& Exclusion Devices: Stromberg's (Catcher-Holder Poles for animal restraint)
Copper mesh hole filler:

Allen Special Products (Stuf-Fit)

Electric fencing:

Gallagher, Poultry Health Services (Gallagher) - Waterford

(ShockTactics, Flexinet) - Lifetime Fence • Gardener's Supply

\section{Live Traps:}

National Live Trap - Reed-Joseph (Trap Away) - Safeguard (Safeguard)

- Tomahawk, Brody, Plow \& Hearth (Tomahawk) - Wildlife Control

- Woodstream, Brody, Great Lakes, Harmony, Peaceful Valley,

Gardener's Supply, Gardenville (Havahart, Victor)

Wildlife Removal/Relocation Services:

Critter Control (for small animals only)

\section{Least-Toxic Chemical Controls}

Repellents:

Lakeshore (Green Screen) • Nixalite, Peaceful Valley, Plow \& Hearth (Ropel) • Gardener's Supply (Champon's)

\section{RODENTS}

\section{Identification \& Monitoring}

Flashlight with red filters:

Streamlight, Brody (Streamlight)

Rodent contamination detection devices:

Brody (Spectronics, Blacklight Kits)

\section{Physical Controls}

Alarms \& detonators:

Bird-X (BirdGard, Transonic IX-L, Ultrason ET)

Reed-Joseph (Scare Away Pyrotechnics, Multi Bang Automatic Cannon, Rotomat Revolving Platform)

Agricultural Supply • Critter Control • Margo

Barriers \& Exclusion Devices:

Harmony (Root Guard Baskets)

Copper mesh hole filler:

Allen Special Products (Stuf-Fit)

Metal mesh barrier:

In-Tex Products (Guard-All)

Tree wrap:

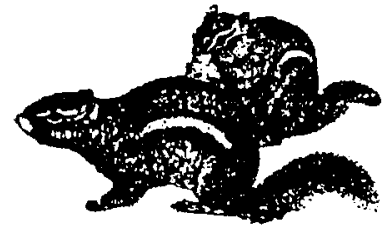

Pest Management (T\&G Plastic Tree Shields)

\section{Traps:}

Sterling (Rescue! Dispose-A-Mouse) • Enforcer (Enforcer Rat \& Mouse Glue Traps)

Glue board trap:

Woodstream (Victor Holdfast) • Bell (Trapper products) • L\&F Products

(d-Con Ultra Set) · JT Eaton (Eaton's Stick-em)

Live traps:

Consep, Gardens Alive!, Harmony (Black Hole Gopher Trap) (Call

Consep for local distributors) - Reed-Joseph (Trap Away) - Seabright,

Phero Tech (Smart Mouse Trap) • Tomahawk, Brody, Plow \& Hearth

(Tomahawk) - Woodstream, Brody, Great Lakes, Harmony, Peaceful

Valley, Gardener's Supply, Gardenville (Havahart, Victor) -

Woodstream (Victor Holdfast) • Brody (Ketch-all, Snap-E, Clean Catch)

- Necessary (Victor Tin Cat) 


\section{Least-Toxic Chemical Controls}

Baits:

Necessary (Rampage)

Balt stations:

Bell, Necessary (Protecta, RTU) • Brody (Magic Box, Eaton, Bell

Protecta)

Stop feed, single dose ( $0.075 \%$ cholecalciferol Vitamin D3):

Bell (Quintox)

Zinc phosphide (2\%):

Bell (ZP Rodent Bait AG)

Repellents:

Nixalite, Peaceful Valley, Plow \& Hearth (Ropel) • Roxide (Revenge

Rodent Smoke Bomb)

\section{SKUNKS}

\section{Identification \& Monitoring}

Skunk Deodorant:

Drs. Foster \& Smith (Skunk Kleen, Outright Skunk Odor Eliminator)

\section{Physical Controls}

\section{Electric Fencing:}

Gallagher, Poultry Health Services (Gallagher) - Waterford

(ShockTactics, Flexinet) • Lifetime Fence • Gardener's Supply

\section{Live Traps:}

National Live Trap - Reed-Joseph (Trap Away) - Safeguard (Safeguard) - Tomahawk, Brody, Plow \& Hearth (Tomahawk) - Woodstream, Brody, Great Lakes, Harmony, Peaceful Valley, Gardener's Supply, Gardenville (Havahart, Victor)

\section{Least-Toxic Chemical Controls}

Repellents:

Peaceful Valley (Ropel)

\section{WILDLIFE}

\section{Physical Controls}

Alarms \& Detonators:

Reed-Joseph (Scare Away Pyrotechnics, Multi Bang Automatic Cannon, Rotomat Revolving Platform) - Agricultural Supply • Critter Control • Margo

Copper Mesh Hole Filler:

Allen Special Products (Stuf-Fit)

Electric Fencing:

Waterford (ShockTactics, Flexinet)

Lifetime Fence

Live traps:

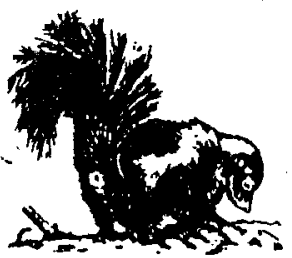

Margo (for large animals) • National Live Trap • Reed-Joseph (Trap Away) - Safeguard (Safeguard) - Tomahawk, Brody, Plow \& Hearth (Tomahawk) - Wildlife Control - Woodstream, Brody, Great Lakes, Harmony, Peaceful Valley, Gardener's Supply, Gardenville (Havahart,
Victor) • Brody (No Odor Skunk Trap)

Wildlife Removal/Relocation Services:

Margo (for large animals) • Critter Control (for small animals only)

\section{Least-Toxic Chemical Controls}

Repellents:

Nixalite, Peaceful Valley, Plow \& Hearth (Ropel)

\section{MISCELLANEOUS}

\section{Educational Materials}

Pest Management Publications \& AV Products:

Integrated pest management publications, slides $\&$ videos: Bio-Integral Resource Center

\section{Identification \& Monitoring}

\section{Information/Identification Services:}

Information, identification of agricultural, urban insects: Cooperative Extension Service (local offices)

Computers, Software, Instruments:

Weather monitor $\&$ disease

forecasting system:

Neogen Corp. (EnviroCaster)

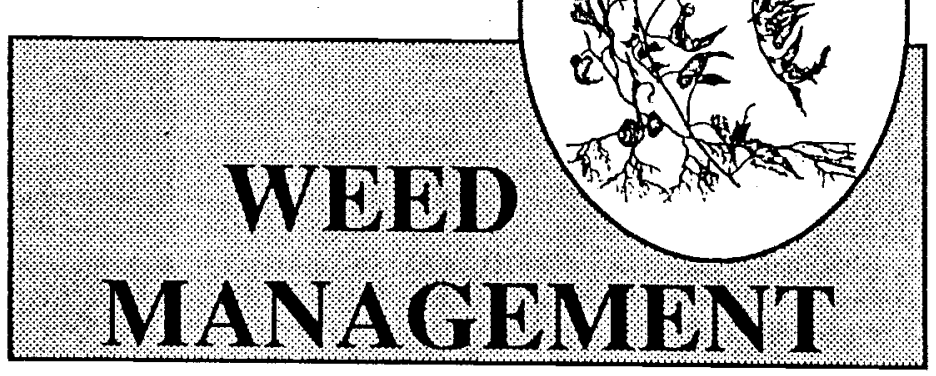

\section{Educational Materials}

Pest Management Publications \& AV Products:

Integrated pest management publications, sildes $\&$ videos: Bio-Integral Resource Center

Slides on biological control of stored grain pests: $\mathrm{BCW}$

\section{Identification \& Monitoring}

Information/Identification Services:

Information, identification of agricultural, urban insects:

Cooperative Extension Service (local of fices) - USDA Biocontrol of Weeds Laboratory

Information service on biological control of weeds:

International Institute of Biological Control

Pesticide detection kits:

Quantix Systems (For atrazine, triazine, alachlor, metolachlor, benomyl, 
chlorothalonil, trifluralin, imazaquin, PAH's)

\section{Physical Controls}

Barriers \& Exclusion Devices:

Aspinalt crack flller:

Garon Products (Crack-Fill)

Mulch:

Peaceful Valley (Hortopaper, IRT, Planter's Paper) • Birkett Mills (MUL-TEX)

Sunlight Barrler (aquatic weeds):

Aquashade (Aquashade)

Weed control fabric:

Easy Gardener (WeedBlock Landscape Fabric) - Reemay (Typar Landscape Fabric) • Gardener's Supply (Weed Mat) - Gardens Alive! •

Flamers:

Ben Meadows - Thermal Weed Control Systems - Forestry Suppliers (Panama) - Plow \& Hearth (Weed Destroyer) - Flame Engineering, Harmony (Red Dragon)

Heat Treatment (system using boiling water):

Waipuna (Waipuna System) (Call BIRC for details on use in the U.S.)

Skin Protectant:

Polson oak/ivy cleanser/prevention:

Apex Medical (Stoko-Gard Outdoor Cream) (Sell only to pharmacists) Interpro (Ivy Shield Prevention Cream) - Smith \& Hawken (Tecnu Cleanser)

\section{Weeding Animals:}

Books, pamphlets, lists of breeders:

American Donkey \& Mule - American

Southdown Breeders' (sheep)

- Natural Colored Wool Growers'

Chickens:

Inman Hatcheries - Murray McMurray Hatchery

- Stromberg's Chicks \& Game Birds Unlimited

Weeding Tools:

Brush-clearing tools: Forestry Suppliers

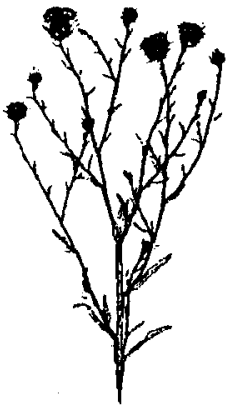

Hand tools: Gardener's Supply - Smith \& Hawken - Plow \& Hearth Peaceful Valley - BCS America - A.M. Leonard - Gardenville - New Tribe (Weed Wrench)

Mechanical harvester: Hockney Underwater Weed Cutter Rotary cutters: Bush Hog (Bush Hog Rotary Cutter)

Shields to protect tree bark from weed trimmer injury: Pest Management (T\&G Plastic Tree Shields)

Tiller/mulcher/mower: Weed Badger (Weed Badger)

Weedeater: Beaird-Poulan/Weed Eater - Homelite Div. of Textron (Brushcutter) (Call Homelite for local distributors) - BeeTee Engine Sales, BecTee Engine Sales (Husqvarna)

\section{Biological Controls}

Sterile grass carp fish (for aquatic weeds):

American Spon (not often available in CA; diploid and triploid) - East Arkansas - Inslee Fish Farm - J.M. Malone (triploid grass carp) - Keo Fish Farms (diploid and triploid) • Richmond Fisheries • Whiskers Catfish Farm (triploid)
Herbivores:

Aceria malherbae (for field bindweed): Praxis

Agapeta zoegana (for knapweed): Praxis

Agrilus hyperici (beetle borer for St. johnswort): Bio Collect

Aphthona spp. (beetle for leafy spurge): Praxis - BCW

Apion fuscirostre (stem weevil for Scotch broom): Bio Collect

Apion ulicis (stem weevil for gorse): Bio Collect

Bangasternus orientalis (for yellow starthistle): Bio Collect - BCW • Peaceful Valley

Ceuthorynchidius horridus (for musk thistle): Praxis

Ceutorhynchus litura (thistle weevil for Canada thistle): $\mathrm{BCW}$ - Praxis Chrysolina quadrigemina (beetle for St. johnswort): Bio Collect - BCW Coleophora klimeschiella (for Russian thistle leafminer): Bio Collect Coleophora parthenica (for Russian thistle stem borer): Bio Collect Hyles euphorbiae (hawk moth for spurge weed): Praxis

Larinus planus (weevil for Canada thistle): BCW

Leucoptera spartifoliella (leafminer for Scotch broom): Bio Collect

Longitarsus jacobaeae (flea beetle for tansy ragwort): $\mathrm{BCW} \cdot \mathrm{Bio}$ Collect

Microlarinus lareynii (weevil for puncturevine): Bio Collect Microlarinus lypriformus (weevil for puncturevine): Bio Collect Oberea erythrocephala (beetle for leafy spurge): BCW - Praxis Phrydiuchus tau (weevil for Mediterranean sage): Bio Collect

Rhinocyllus conicus (weevil for musk, milkthistle, Italian thistle): Bio Collect $\cdot \mathrm{BCW} \cdot$ Praxis

Sphenoptera jugoslavica (stem borer for knapweed): Praxis Spurgia esulae (gall midge for leafy spurge): Praxis Urophora affinis (gall fly for knapweed): $\mathrm{BCW}$ Urophora cardui (gall fly for Canada thistle): BCW Urophora quadrifasciala (gall hy for knapweed): $\mathrm{BCW}$ Urophora sirunaseva (gall fly for yellow starthistle): Bio Collect Urophora spp. (gall fly for Canada thistle, knapweed): Praxis Trichosirocalus horridus (weevil for musk thistle): BCW - Praxis Tyria jacobaeae (cinnabar moth for tansy ragwort): Bio Collect $•$ BCW Zeuxidiplosia giardi (for St. johnswort): Bio Collect

Microbial Herbicides:

Aeschynomene virgica (for nor thern jointvetch): Ecogen (Collego)

Distributes stem \& seed weevils against puncture vine, other beneficials:

CALTEC Agri Marketing Services

Weeder Geese (brown Chinese):

Holderread's Waterfowl Farm \& Preservation Center • Morris Farms \& Hatcheries

\section{Least-Toxic Chemical Controls}

Application equipment:

Forestry Suppliers (Hypo-Hatchet Tree Injectors) • AG Spray Equipment - Chemihoe (Chemihoe for spot treatments)

\section{Herbicidal Soap:}

Mycogen, Charley's (Sharpshooter Herbicide Concentrate) - Mycogen (DeMoss) - Ringer, Bozeman, Gardens Alive!, Harmony, IFM, The Natural Gardening, Necessary, Nitron, Peaceful Valley (SuperFast Weed and Grass Killer -name changed from Sharpshooter in 1993) - Ringer (Moss Attack) 


\section{ADDRESSES}

3M Corp, 3M Center, St Paul, MN 55133; 612/733-1110

Abbott Laboratories, 1400 Sheridan Rd. N., Chicago, IL 60064; 800/323-9597

Abraxas Communications Ltd., 118810 Kingsway Ave., Edmonton, Alb. CANADA T5G 0X5; 403/425-8957

agAccess, PO Box 2008, Davis, CA 95616; 916/756-7177, Fax 916/756-7188

Agape Farms, 29 Ironia Rd., Mendham, NJ 07945; 201/543-5992, Fax 201/543-0648

AgBloChem Inc., 3 Flectwood Ct., Orinda, CA 94563; 510/254-0789.

AgBio Development Inc., 9915 Raleigh Street, Westminister, CO 80030; 303/469-9221, Fax 303/469-9598

Agricola "El Sol", 30 Calle 11-42, Zona 12, GUATEMALA, C.A.; Tel. 760496

Agricultural Supply, 1435 Simpson Way, Escondido, CA 92029; 619/741-0066

AgriDyne Technologies Inc., 417 Wakara Way, Salt Lake City, UT 84108; 800/657. 3090, 801/583-3500

AgriSense, 4230 W. Swift, Ste. 106, Fresno, CA 93722; 800/756-2847

Agrl-Systems International, 238 O.W. Rd., Bangor, PA 18013; 215/363-6700, Fax 215/ 863-4622:

Agro-Chem Corp., 11150 W. Addison St., Franklin Park, IL 60131; 708/455-6900

AG Spray Equlpment, 1521 Industrial Dr., Hoppkinsville, KY 42240; 502/886-0296

A.I. Root Co., PO Box 706, 623 W. Liberty St., Medina, OH 44258; 216/725-6677

Allen Special Products Inc., PO Box 605, Montgomeryville, PA 18936; 800/848-6805, 215/362-7515, Fax 215/997-6654

Allergy Control Products Inc., 96 Danbury Rd., Ridgefield, CT 06877; 800/422-DUST

American Agrifabrics, 8607 Roberts Dr., Ste. 200, Atlanta, GA 30350; 404/594-0699, Fax 404/594-0665

Amerlcan Donkey \& Mule Society, 2901 North Elm St., Denton, TX 76201; 800/5844002, 817/382-6845

Amerlcan Entomologlcal Institute, $3005 \mathrm{SW}$ 56th Ave., Gainesville, FL 32608; 904/3776458, Fax 904/377-6458

American Southdown Breeders' Asśoc., HCR 13, Box 220, Freedonia, TX 76842; 915/429-6226

American Sport Fish Hatchery, PO Drawer 20050, Montgomery, AL 36120; 205/281. 7703, Fax 205/288-6376

A.M. Leonard, Inc., 6665 Spiker Rd., PO Box 816, Piqua, OH 45356; 800/543-8955, Fax 800/433-0633
Amos Rubin (Int.), 14, Rahavat Ilan, Givat Shmuel 51905, ISRAEL; 972-3-5351798. Fax 972-3-5341019

AnImal Repellents Inc., PO Box 999, Griffin, GA 30224; 800/241-5064

Apex Medical, PO Box 1235, Sioux Falls, SD 57101; 800/328-2935

Applied Bionomics Ltd., 11074 W. Saanich Rd., Sidney, BC CANADA V8L 5P5; 604/ 656-2123, Fax 604/656-3844

Aquashade Inc., $60120 \mathrm{~W}$. Douglas Ave., Milwaukee, WI 53218; 800/558-5106, Fax 414/438-5671

Aquatrols, 5 North Olney Ave., Terry Hill, NJ 08003; 800/257-7797, 609/751-0309

ARBICO, PO Box 4247 CRB, Tucson, AZ 85738; 800/767-2847, 602/825-9785, Fax 602/825-2038

Assoclation for Sensible Pest Control, PO

Box 201, Clayton, CA 94517-0201

ATI Plastics, 4405 101st Ave., Edmonton,
Alb. CANADA T5J 2K1; 800/661-3606

Automata Inc., 16216 Brooks Rd., Grass Valley, CA 95945-8816; 916/273-0380, Fax 916/273-0381

Azienda Agrarla Giardina, S.P. 104, Contrada Carrozzieri Milocca, 90600 Siracusa, ITALY; 0931-721444

B\&G Equipment Company, P.O. Box 130 , Plumsteadville, PA 18949-0130; 215/7668811, Fax 215/766-8240

Baley's, 1206 N. 31st Ave., Melrose Park, IL 60160; 708/681-0900, Fax 708/544-3204

Barmann, Fabricius Str. 2, 4010 Hilden, GERMANY; 02103-51233

Bassett's Cricket Ranch Inc., 365 S. Mariposa, Visalia, CA 93292; 209/747. 2738, Fax 209/74-3619

Bat Conservation International, PO Box 162603, Austin, TX 78716-2603; 512ß327. 9721, Fax 512/327-9724

Bay Area Bat Protection, 1312 Shiloah Rd.,

\section{KOPPERT DISTRIBUTORS ADDRESSES}

Agrofresas S.A., Chalct El Pozuclo, Ctra. Rábida - Moguer, Apartado de Correos 71, 21800 Moguer, SPAIN; 55-372443 Aster Devrleze N.V., Molenstraat 2, 2638

Reet, BELGIUM; 03-8884090

Casteels N.V., Mechelbaan 73, 2861

O.L.Vr. Waver, BELGIUM; 015 755529

Complejo Asgrow Semillas S.A., Zurbano 67-2 B, 28010 Madrid, SPAIN; 1 4420399

EcoTrade, Netolická 11, 37012 Ceské Budejovice, CZECHOSLOVAKIA; 3840260, Fax 38-43861

G.A.M. van der Goes, Georg Hench Strabe 13-2, 8400 Nümberg 80 , GERMANY; 011-328062

G.F. Garta A/S, Nordholmen 5, Postboks 1130, 2650 Hvidovre, DENMARK; 31 491144 , Fax 31491558

Gerhart, Inc., 6346 Avon Belden Rd., N Ridgeville, OH 44039; 216/327-8056. Fax 216/353-9457

Harantonis, 58500 Skydra, Pellis, GREECE; 381-89802

J. Mertens B.V., Vergelt 3, Postbus 8319, 5990 AA Baarlo, Neiderlande, GERMANY; 04707-9292, Fax 04707-3045

Koppert France S.A.R.L., Lot. Ind. du Puits des Gavottes , 147 Avenus des Banquets, 84300 Cavaillon FRANCE; 90783013

Koppert Hungaria Kft., Moricz Zs. u.
11., 2500 Esztergom, HUNGARY; 3633-313240

Koppert Italia S.r.l., Via Porrettana Nord 37/2, 40043 Marzabotto (BO), ITALY; 051-931346, Fax 051-931346

Leu + Gygax AG, Fellstrasse 1, Postfach 30, 5413 Birmenstorf AG, SWITZER-

LAND; 056-851515

Neoquímica, Av. Defensores de Chaves 35-6, 1000 Lisbon, PORTUGAL; 01 548417, Fax 01-3560230

Olcosa, La Moura 38, 15008 La Coruña, SPAIN; 81-253928

Oy Schetellg AB, Martinkylantie 52, 01720 Vantaa, FINLAND; 0-852061

R. Bässler, Universität Hohen heim, 305 Versuchsstation fur Gartenbau, 70593 Stuttgart, GERMANY; 0711-4593080, Fax 0711-4593750

Safer Ltd., 3 Pullman Ct., Scarborough. Ont. CANADA MIX 1E4; 800/3875306 (in Canada), 416/291-8150, Fax 416/291-1755

Stan Brouard Ltd., PO Box 383, Landes du Marche, Vale, Guemsey, CHANNEL ISLANDS; 0481-52521

Svenska Predator AB, Knut Pais Vag 8, Box 14017, 25014 Heisingborg, SWEDEN; 042-201130

TukoGardenia Oy, Kytomaantic 14, 04200 Kerava, FINLAND; 0-6191

Zaadhandel Hollandla N.V., Westkaai 8. 2170 Merksem, BELGIUM; 03-6470607 
Sturgeon Bay, WI 54235; 414/743-9049

BCS America Inc., 13601 Providence Rd., Matthews, NC 28105-7729; 704/846-1040

(BCW) Blological Control of Weeds, 1418 Maple Dr., Bozeman, MT 59715;406/5865111

Beacon Dogs Inc., 1409 Bay Head Rd., Annapolis, MD 21401; 410/757-4999

Beaird-Poulan/Weed Eater, PO Box 9329. Shreveport, LA 71139-9329; 318/683-3596

Beaver Plastics Ltd., 12150160 St., Edmonton, Alb. CANADA T5V 1H5; 403/ 453-5961, Fax 403/453-3955

BeeTee Engine Sales, 21075 Alexander Ct. Unit H, Hayward. CA 94540; 510/887-8301

Bell Laboratories Inc., 3699 Kinsman Blvd., Madison, WI 53704; 608/2A1-0202, Fax 608/241-9631

Beneficlal Insectary, 14751 Oak Run Rd., Oak Run, CA 96069; 800/477-3715,916/ 472-3715, Fax 916/472-3523.

Beneficial Resources, Inc., PO Box 34, Tucbotville, PA 17772; 717/649-6289, Fax 717/649-5162

Ben Meadows Co., PO Box 80549, Atlanta, GA 30366; 800/241-6401, 404/455-0907, Fax 800/628-2068

Better Yleld Insects, RR 3, Site 4, Box 48, Belle River, Ont. CANADA NOR 1A0; 519/ 727-6108, Fax 519/727-5989

BInab Blolnovation AB, Bredholmen Box 56, S-54242, Algaras, SWEDEN; 46-506-42072, Fax 46-506-42072

Blo-Agronomics, PO Box 1013, Clovis, CA 93613; 209/297-9288

Bio Ag Services, 4218 W. Muscat, Fresno, CA 93706; 209/268-2835

BioBase Technologies, Inc., RD 1 Box 715, Landisburg, PA 17040; 7 17/789-3434

Biobest, Ilse Velden, 18, 2260 Westerlo, BELGIUM; 32-14-231701, Fax 32-14231831

Blo Collect, 5481 Crittenden St., Oakland, CA 94601; 510/436-8052, Fax 510/532-0288

Blocontrol Ltd., PO Box 515, Warwick, Queensland, AUSTRALIA 4370; 076/ 614488

Biofac, Inc., PO Box 87, Mathis, TX 78368; 512/547-3259, Fax 512/547-9660

Bio-Integral Resource Center, PO Box 7414, Berkeley, CA 94707; 510/524-2567, Fax 510/524-1758

Biolab Centrale Ortofrultticola, Via Masiera I, 1191; 47020 Cesena (FO), ITALY; 0547380637, Fax 0547-380795

BioLogic, PO Box 177, Willow Hill, PA 17271; 717/349-2789, Fax 717/349-2789

Biological Control Insectarles, Kibbutz Sde Eliyahu, D.N. Bet-Shean, ISRAEL 10810; 06-580904-6, Fax 06-587090
Biological Control of Weeds, 1418 Maple Dr. Bozeman, MT 59715; 406/586-5111. Sec BCW

Biological Crop Protection, Ltd., Occupation Rd., Wye, Nr. Ashford Kent TN25 5AH, UNITED KINGDOM; 0233-813240, Fax 0233-813383

BioQuip Products, 17803 La Salle Ave., Gardena, CA 90248-3602; 310/324-0620. Fax 310/324-7931

BioShield, PO Box 9068, Fresno, CA 93790; 209/226-7463, 209/297-7480

Blosys Corp., 1057 East Meadow Circle, Palo Alto, CA 94303; 800/821-8448, 415/8569500

Biotactics, Inc., 425 La Cadera Dr. W., Ste. 10D, Riverside, CA 92501; 909/320-1366, Fax 909/781-6572

Biotechnlk, Winnckendonker Str. 43, (Potthaus) D 4178 Kevelaer 2, GERMANY; 01149-2825/6571, Fax 01149-2825/1776

B1-Pro Industries Inc., PO Box 998-845, El Segundo, CA 90245-3060; 800/634-7888, 310/476-0250

BIrd-X Inc., 730 West Lake St., Chicago, IL $60661 ; 800 / 662-5021,312 / 648-2191$, Fax 312/648-0319

Birkett Mills, PO Box 440, Penn Yan, NY 14527; 315/536-3311, Fax 315/536-6740

Bluebird Enterprises, 6408 South Fig, Fresno, CA 93706; 209/268-1200

Blue Diamond Exterminating Inc., Hwy. 421 S., Hyden, KY 41749; 606/672-3880

BoBiotrol, 54 South Bear Creek Dr., Merced, CA $95340 ; 209 / 722-4985,209 / 358-1488$, Fax 209/358-1488

Bon-Aire Industries, Inc., 3240 Industry Dr., PO Box 490, Signal Hill, CA 90801

Bozeman Bio-Tech, Inc., PO Box 3146, 1612 Gold Ave., Bozeman, MT 59772; 800/2896656, 406/587-5891, Fax 406/587-0223

Brody Enterprises Inc.; 9 Arlington Place, Fair Lawn, NJ 07410; 800/458-8727, 201/ 794-3616

Brushy Mountain Bee Farm, Inc., Route 1, Box 135, Moravian Falls, NC 28654

Budal Cs, Hodmesvasarhely Inst. of Soll \& Crop Protection Service, PO Box 99, 6801, Hodmesvasarhely, HUNGARY; 46-611

Buena Blosystems, PO Box 4008, Ventura, CA $93007 ; 805 / 525-2525$, Fax 805/5256058

Bugs for Bugs, 28 Orton St., Mundubbera 4626, AUSTRALIA; 61-71-654663, Fax 6171-654626

Bunting Biological, PO Box 2430, Oxnard, CA 93034-2430; 805/986-8265, Fax 805/ 986-8267

Bunting Biological Control Ltd., Great Horkesley, Colchester, Essex C06 4AJ,
UNITED KINGDOM; 0206-271300, Fax 0206-272001

Bush Hog, PO Box 1039, Selma, AL 367021039; 205/872-6261, Fax 205/872-0168

BWS Distributors Inc., 1847 Piner Rd., Santa Rosa, CA 95403; 800/862-4685

C. A.B. International Institute of Parasitology, 395A Hatfield Rd., St. Albans, Herts. ALA OXU, UNITED KINGDOM; (0727) 33151

Cabot Corp., PO Box 188, Tuscola, IL 61453; 217/253-3370

California Statewide IPM Project, $2101 \mathrm{C}$ Wuickson Hall, University of Calfornia, Davis, CA 95616; 916/752-8350

CALTEC Agri Marketing Services, PO Box 576155, Modesto, CA 95357; 800/491 BUGS, 209/575-1295, Fax 209/575-0366

Canadian Insectaries, 5 Alderwood Rd., Winnipeg, Man. CANADA R2J 2K7; 204/ 257-3775, Fax 204/256-2206

Carter-Wallace, Inc., 1345 Avenue of the Americas, New York, NY 10105; 212/339. 5000

Cat Claw Inc., PO Box 3778, Johnstown, PA 15904; 800/832-2473, Fax 814/269-3800

Center Laboratories Inc., 35 Channel Dr., Port Washington, NY 11050; 800/223-6837, Fax 516/767-4229

Central Coast Insectary, 391 Hames Rd., Watsonville, CA 95076-0234; 408/7261853, Fax 408/726-5123

Charley's Greenhouse Supply, 1569 Memorial Hwy., Mt. Vernon, WA 98273; 800/322-4707

Chemihoe Inc., PO Box 387, Plainview, TX 79073; 806/293-3553

C. H. Musgrove, 2901 Everwood Dr., Riverside, CA 92503; 909/785-1680

Chr. Hansens Bio Systems, Boge Alle 10-12, DK-2970 Horsholm, DENMARK; 45-45-7666-66, Fax 45-45-76-60-66

Ciba Geigy/Plant Protection Division, PO Box 18300, Greensborough, NC 274198300; 919/632-6000

CIE La Croix, Rue du Pont, 21 Plougastel, Daoulas, FRANCE 29213; 98-403030, Fax 98-042437

Clark Mosquito Control, PO Box 72288, Roselle, IL 60172; 708/894-2000

Clark Pest Remedy, PO Box 1031, McDonough, GA 30253; 404/957-1915

Clorox Company, 3030 LBJ Freeway, Ste. 1220, LB38, Dallas, TX 75234; 214/4846326, Fax 214/406-0496

Coast Agri-Protect Dept., RR\#2, 464 Riverside Rd. S., Abbotsford, BC CANADA V2S 4N2; 604/853-4836

Cochranes of Oxford, Ltd., Leafield Witney, Oxfordshire, UNITED KINGDOM OX85NY 
CO. DEA, CREAT del Chambre d'Agriculture, Quartier la, Baronne, La Gaude, FRANCE 06610;

Combined Scientific Supplles, PO Box 1446, Fort Davis, TX 79734; 915/426-3851

Conrad Appel, Bismarckstr. 5g, 6100 Darmstadh, GERMANY; 06151-852200

Consep Membranes Inc., 213 SW Columbia St., Bend, OR 97702; 800/367-8727, 503/ 388-3688, Fax 503/388-3705

Consulting Dlagnostic Service, 992 Santa Barbara Rd., Berkeley, CA 94707; .510/5249476

Copper Brite Inc., PO Box 50610, Santa Barbara, CA 93150-0610; 805/565-1566, Fax 805/565-1394

Cornflower Farms, PO Box 896, Elk Grove, CA 95759; 916/689-1015, Fax 916/689. 1968

Coverplus, Hyde, Cheshire, UNITED KINGDOM

Critter Control, 640 Starkweather, Plymouth, MI 48170; 313/453-6300

CRS Company, 2909 NE Anthony Ln., St. Paul, MN 55119; 612/571-7060

CSI, One Woodlawn Green, Ste. 250, Charlotte, NC 28217; 800/421-8661, 704/ 522-0825, Fax 704/527-8232

CTIF L/SAP, Centre de Balandran, 30127 Bellegarde, FRANCE

Custom Copper, PO Box 4939, Ventura, CA 93004; 805/647-1652,206/676-5969

Cyanamid, Agricultural Products Division, One Cyanamid Plaza, Wayne, NJ 074708426; 201/831-2087

Dadant \& Sons, 51 S. 2nd St., Hamilton, IL 62341-1399; 800/637-7468, 217/847-3324, Fax 217/847-3660

De Groene Vlieg, Duivenwaardsedijk 1, 3244 LG Nieuwe Tonge, THE NETHERLANDS; 01875-1862, Fax 01875-2445

Degussa Corp., 65 Challenger Rd., Ridgefield Park, NJ 07660; 800/237-6745, 201/641 6100

Delmhorst Instrument Co., 1018 6th St., Towaco, NJ 07082; 201/334-2557

Del Pharmaceuticals, Inc., 565 Broad Hollow Rd., Farmingdale, NY 11735; 516/293-7070, Fax 516/293-1515

D. Gilbert Industries, 5611 Krueger Dr., Jonesboro, AR 72401; 800/643-0400, 501/ 932-6070, Fax 501/932-5609

Diacide Inc., 1016 6th St., Nevada, IA 50201; 800/767-6222

Drs. Foster and Smith, Inc., 2253 Air Park Rd., PO Box 100, Rhinelander, WI 54501; 800/826-7206

Duclos Agro-Biotech S.A., B.P. 3, 13240 Septemes les, Vallons, FRANCE; 91 963000, Fax 91-962527
D-VAC Company, PO Box 2506, Ventura, CA 93002; 805/643-5407, Fax 805/643-6267

Earth Tools, 9754 Johanna P1., Shadow Hills, CA 91040; 800/825-6460, 818/353-5883, Fax 818/353-8966

East Arkansas Fish Distributors, PO Box 361, Hazen, AR 72064; 501/255-3455

Easy Gardener, PO Box 21025, Waco, TX 76702-1025; 800/327-9462, 817/753-5353

Ecogen Inc., 2005 Cabot Blvd. West, Langhome, PA 19047-1810; 215/757-1590, Fax 215/757-2956

Ecohealth Inc., 110 Broad St., Boston, MA 02110; 617/742-2400, Fax 617/350-6260

EcoSafe Products Inc., PO Box 1177, St. Augustine, FL 32085; 800/274-7387, 904/ 824-5884, Fax 904/824-5100

EcoSclence, Inc., One Innovation Dr., Worcester, MA 01605; 508/754-0300

Electrostatic Spraying Systems, Inc, PO Box 151, Watkinsville; GA 30677; 706/7690025, Fax 706/769-8072

Enforcer Products, Inc., PO Box 1068, Cartersville, GA 30120; 800/241-5656, 706/ 386-0801, Fax 706/386-1659

English Woodlands Biocontrol, Hoyle Depot, Graffham Petworth, West Sussex, GU28 OLR, UNITED KINGDOM; 07986-574

Entosphere, Inc., 33253 Ave. 9, Madera, CA 93638

Eriez Magnetics Pty. Ltd., PO Box 82, Terry Hills, AUSTRALIA (NSW) 2084; 02/4501988

ETEX Ltd., 3200 Polaris Ave. \#9, Las Vegas, NV 89102; 800/543-5651, 702/364-5911. Fax 702/364-8894

ETS Rene Briand, La Gibraye, 44430 Saint, Sebastien sur Loire, FRANCE

Fairfax Blological Laboratory Inc., PO Box 300, Clinton Comers, NY 12514; 914/2663705, Fax 914-266-5390

FAR (Foothill Agricultural Research), 510$1 / 2$ West Chase Dr., Corona, CA 91720; 909/371-0120, Fax 909/279-5150

Farnam Co., PO Box 12068, Omaha, NE 68112; 602/285-1660, Fax 602/285-1803

Fibertron Inc., 1321 Valwood Pkwy., Bldg. 440, Carrollton, TX 75006; 214/406-8881, Fax 214/406-8882

First Choice Manufacturing Ltd., 1940256 th Ave., Surrey, BC CANADA V3S 6K4; 604/ 534-8626

Fisons Corp., 755 Jefferson Rd., Rochester, NY 14623; 800/234-5535, 716/475-9000

Flame Engineering Inc., PO Box 577. LaCrosse, KS 67548; 800/255-2469, 913/ 222-2873, Fax 913/222-3619

Flea Busters, 6555 Northwest 9th Ave., Ste. 412, Fort Lauderdale, FL 33309; 800/666$3532,213 / 470-3532$ (in LA)
Foothill Agricultural Research, 510-1/2 West Chase Dr., Corona, CA 91720; 909/3710120, Fax 909/279-5150. See FAR

Forestry Suppllers Inc., 205 W. Rankin St., Jackson, MS 39201-6126; 800/647-5368, 601/354-3565

Gallagher Power Fence, 18940 Redland Rd., PO Box 708900, San Antonio, TX 78270; $800 / 531-5908$

Gardener's Supply Co., 128 Intervale Road, Dept. GRSM, Burlington, VT 05401; 802/ 863-1700, Fax 802/660-3501

Gardens Alive!, 5100 Schenley Place, Lawrenceburg, IN 47025 ; $812 / 537-8650$ or 812/537-8652, Fax 812/537-8660

Gardenville of Austin, 8648 Old Bee Caves Rd., Austin, TX 78735; 512/288-6115, Fax 512/288-6114

Garlic Research Labs, 3550 Wilshire Blvd., Ste. 200. Los Angeles, CA 90010; 213/386-5300

Garon Products Inc., PO Box 1924, Wall, NJ 07719-1924; 800/631-5380, 201/449-1776

Gartnerel Hatto Welte, Maurershorn 10, 7752 Insel Reichenau, Bodensee, GERMANY; 07534-7190, Fax 07534-1458

GIBCO BRL, 8400 Helgerman Court, Gaitherburg, MD 20877; 800/828-6686, 301-840-8000, Fax 800/331-2286

Grace-Slerra, PO Box 4003, Milpitas, CA 95035; 800/492-8255, 408/263-8080, Fax 408/262-9344

Great Lakes IPM: 10220 Church Rd. NE, Vestaburg, MI 48891; 800/235-0285, 517/ 268-5693, Fax 517/268-5311

Green Pro Servlces, 380 S. Franklin St., Hempstead, NY 11550; 800/645-6464, 516/ 538-6444, Fax 516/538-2042

GTF Labs, PO Box 2646, Fresno, CA 93745; 209/268-3417

Guardian Spray Corp., PO Box 40121, Bakersfield, CA 93384; 805/323-4412

Gustaison, Inc., PO Box 660065, Dallas, TX 75266-0065; 214/985-8877

Hansens Bio Systems, Boge Alle 10-12, Horsholm, DENMARK DK-2970; Fax 01142-76-54-55

Hanisen's Pet Systems, PO Box 0024 , Newport Beach, CA 92660; 800/HPS-5735, 714/960-7549, Fax 714/846-4080

Harmony Farm Supply, 3244 Hwy. 116, \#B, Sebastopol, CA 95472; 707/823-9125, Fax 707/823-1734

Hawkesbury Integrated Pest Management, PO Box 436, Richmond, NSW 2753 , AUSTRALIA; 045/701331, Fax 045/701314

Health Enterprises Inc., 90 George Levin Dr., N. Attleboro, MA 02760; 800/633-4243

Hercon Environmental, Aberdeen Rd., Emigsville, PA 17318; 717/764-1191, Fax 717/764-5211 
Herltage Rosarium, 211 Haviland Mill Rd., Brookville, MD 20833; 301/774-2806

Hess \& Clark, Inc., 7th and Orange Streets, Ashland, OH 44805; 800/992-3594

High Country Rosarium, 1717 Downing St., Denver, CO 80218; 303/832-4026, Fax 303/ 832-4026

Hockney Underwater Weed Cutter Co., PO Box 1000, 913 Cogswell Dr., Silver Lake, WI 53170; 414/889-4581

Hogil Pharmaceutical Corp./Interplex Labs, One Byram Brook Place, Armonk, NY 10504; 800/PET-COMB, 914/273-9666, Fax 914/273-9743

Holderread's Waterfowl Farm \& Preservation Center, PO Box 492, Corvallis, OR 97339; 503/929-5338

Homelite Div. of Textron Inc., 14401 Carowinds Blvd., Charlotte, NC 28273; 704/ 588-3200, Fax 704/588-0926

Hortica Inc., RR 1, 723 Robson Rd., Waterdown, Ont. CANADA LOR 2H1; 416/ 689.6984 or 689.3002

Hydro-Gardens, Inc., PO Box 25845 , Colorado Springs, CO 80936; 800/634-6362, 719/495-2266, Fax 719/531-0506

IFM, 333 Ohme Gardens Rd., Wenatchee, WA 98801; 800/332-3179, Fax 509/662-3179

Igene Blotechnology Inc., 9110 Red Branch Rd., Columbia, MD 21045; 800/346-6421, 410/997-2599. Fax 410/730-0540

Industrial Narcọtic Discovery Systems, Inc., PO Box 130, Kenner, LA 70063; 504/4669964

Inman Hatcheries, PO Box 616, Aberdcen, SD 57402-0616; 800/843-1962

Insects Limited, 10540 Jessup Blvd., Indianapolis, IN 46280-1438; 800/992-1991

Inslee Fish Farm, Inc., PO Box 207. Connerville, OK 74836; 405/836-7150

Intagra, 8500 Pillsbury Ave. S., Minneapolis, MN 55420; 800/468-2472, 612/881-5535, Fax 612/881-7002

International Institute of Biological Control, Silwood Park, Buckhurst Rd., Ascot, Berks. SL5 TTA, UNITED KINGDOM; (0344) 872999

International Institute of Entomology, 56 Qucens Gate, London SW7 5JR, UNTTED KINGDOM; 071/584-0067, Fax 071/581 1676

Internatlonal Mycological Instltute, Bakeham Lane, Egham, Surrey TW20 9TY, UNITED KINGDOM; (0784) 470-111, Fax (0784) 470-909

InterNet Inc., 2730 Nevada Ave. N., Minneapolis, MN 55427; 800/328-8456, Fax 612/ 541-9692

Interpro Inc., PO Box 1823, Haverhill, MA $01831 ; 800 / 456-6489$
In-Tex Products Inc., PO Box B, Cleveland, NY 13042; 315/675-8471

IPM Laboratories Inc., PO Box 300, Locke, NY 13092-0300; 315/497-3129, Fax 315/ 497-3129

Isothermics, PO Box 6951, Orange, CA 92613-6951; 714/974-0951

J.M. Malone \& Son Enterprises, PO Box 158 , Lonoke, AR 72086; 501/676-2800, Fax 501/ 676-2910

Johnny's Selected Seeds, 305 Foss Hill Rd., Albion, ME 04910; 207/437-9294, 207/437. 4301, Fax 207/437-2165

J.P. Kass, Degearweg 65, 2461 TT Langeraar, THE NETHERLANDS; 31-1723-7859

J. Rellly, 5000 Trenton St., Metairic, LA 70003; 504/887-3666

J.T. Eaton \& Company, Inc., 1393 E. Highland Rd., Twinsburg, OH 44087; 800/ 321-3421, Fax 216/425-8353

KemIra Oy/Kemira Blotech, PO Box 330, 00101 Helsinki, FINLAND; 358-0-13211, Fax 358-0-694-1375

Keo Fish Farms, Inc., PO Box 123, Keo, AR 72083; 501/842-2872, Fax 501/842-2156

King \& Kienlen, 6325-C McDonough Dr., Norcross, GA 30093; 404/441-2836, Fax 404/441-9260

Koppert B.V., Veilingweg 17 PO Box 155, 2650 AD Berkelen, Rodenrijs, THE NETHERLANDS; (31) 1891-40444, Fax (31) 01891-15़203

Kuida Ag Supply Co., 14715 S. Mariposa Ave., Gardena, CA 90247; 310/378-5137 or 408/758-9933, Fax 310/378-1015

Kunafin Trichogramma Insectaries, Rtc. 1 Box 39, Quemado. TX 78877; 800/832$1113,210 / 757-1181$, Fax 210/757-1468

KWS, Postfach 146, 3352 Einbeck, GERMANY; 05561-311390, Fax 05561-311322

Lab Safety Supply, PO Box 1368, Janesville, WI 53547-1368; 800/356-0783, 800/3562501, Fax 800/543-9910

Ladd Research Industries Inc., PO Box 1005, Burlington, VT 05402; 802/878-6711, Fax 802/878-8074

Lakeshore Enterprises, 2804 Benzic Hwy., Benzonia, MI 49616; 800/968-9453, 616/ 882-9601, Fax 616/882-5242

L\&F Products, Household Products Div, 225 Summit Ave., Montvale, NJ 07645

Lifetime Fence Co., Inc., 2 Paisley Industrial Park, Carmichael, PA 15320; 412/966-2612

Live Oak Structural, 801 Camelia \#B, Berkeley, CA 94710; 510/524-7101

Lofts Seed Co., PO Box 146, Bound Brook, NJ 08805; 800/526-3890

M \& R Durango Inc., PO Box 886, Bayficld, CO 81122; 800/526-4075, 303/259-3521. Fax 303/259-3587

Margo Horticultural Supplies, Ltd., RR6.
Site 8, Box 11, Calgary, Alb. CANADA T2M 4L5 ; 403/285-9731

McCluney Manufacturing Inc., 28

Morehouse Dr., Freedom, CA 95076; 408/ 722-2194

Merck Ag Vet Division, PO Box 2000, Rahway, NJ 07065; 908/594-4000

MicroFlo Co., PO Box 5948, Lakeland, FL $33807 ; 800 / 451-8461 ; 800 / 543-3120$ (in CA), 813/647-3609, Fax 813/647-3412

Miles Allergy Products, 3525 Regal St., PO Box 3145, Spokane, WA 99220; 509/489. 5656

Miller Chemical \& Fertilizer Corp., PO Box 333. Hanover, PA 17331; 800/233-2040, 717/632-8921

Mine Safety Appliance Co., 5060 Commercial Circle, Unit D, Concord, CA 94520; 800/672-2222,412/967-3000

Mission Pest Control, 23286 Del Lago Dr., Laguna Hills, CA; 92653, 714/586-3799

Monterey Lawn \& Garden Products Inc., PO Box 5317, Fresno, CA 93755; 209/2254770

Morris Farms \& Hatcheries, Inc., 18370 232nd St., Goulds, FL 33170; 305/248-5589

Murray McMurray Hatchery, Wcbster City, IA 50595-0458; 515/832-3280

Mycogen Corp., 4980 Carroll Canyon Rd., San Dicgo, CA 92121; 800/745-7476, 619/ 453-8030

National Live Trap Corp., PO Box 302, Tomahawk, WI 54487; 715/453-2249

National Pediculosis Association, PO Box 149, Newton, MA 02161; 800/446-4672, 617/449-6487, Fax 617/449-8129

Natural Colored Wool Growers' Assoc. 3012 Sutton Rd., Lapecr, MI 48446; 313/ 797-4155

Natural Insect Control, RR\#2, Stevensville, Ontario CANADA LOS 1S0; 416/382-2904, Fax 416/382-4418

Natural Insecto Products, Inc., 630 North Eckhoff St., Orange, CA 92668; 800/3322002, 714/939-9192, Fax 714/939-9548

Natural Pest Control Ltd., Yapton Road, Barnham, Bognor Regis, West Sussex, UNITED KINGDOM PO22 OBQ; Yapton (0243) 553250, Fax 0243552879

Natural Pest Controis, 8864 Little Creek Dr., Orangevale, CA 95662; 916/726-0855

Nature House Inc., Purple Martin Junction, Griggsville, IL 62340; 217/833-2393

Nature's Alternative Insectary, Box 19 Dawson Rd., Nanoose Bay, BC CANADA VOR 2R0; 604/468-7912, Fax 604/468-7912

Nature's Control, PO Box 35, Medford, OR 97501; 503/899-8318, Fax 503/899-9121

Nature's Touch, 11150 W. Addison St., Franklin Park, IL 60131; 708/455-8600 


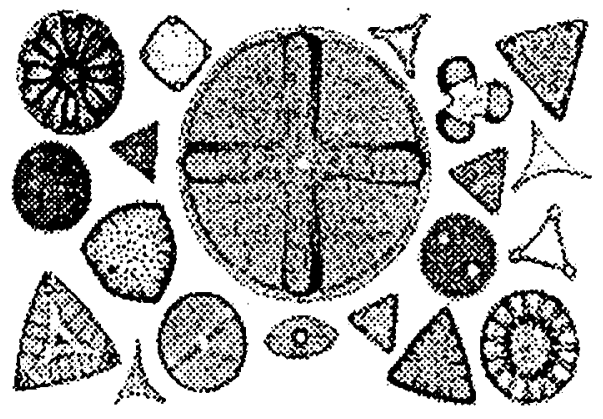

Necessary Trading Co., PO Box 305, New Castle, VA 24127-0305; 800/447-5354, 703/ 864-5103, Fax 703/864-5186

Nematec, PO Box 93, Lafayette, CA 945490093; 510/735-8800

Neogen Corp., 620 Lesher Place, Lansing, MI 48912; 800/234-5333, 517/372-9200, Fax 517/372-2006

New BloProducts Inc., 4737 N.W. Elmwood Dr., Corvallis, OR 97330; 503/752-2045

New Earth Resources, Inc., 826 10th St., Huntington, WV 25701; 800/576-5033

New Era Farm Service, 23004 Rd. 140, Tulare, CA 93274; 209/686-3833

New Tribe, 5517 Riverbanks Rd., Grants Pass, OR 97527; 503/476-9492

Nichols Net \& Twine Co., 2200 Hwy 111, Granite City, IL 62040; 618/797-0211

NISUS Corp.; 101 Concord St. N., Knoxville, TN 37919; 800/264-0870, 615/637-1226, Fax 615/637-2588

Nitron Industries Inc., PO Box 1447 , Fayetteville, AR 72702; 800/835-0123, 501/ 750-1777, Fax 501/750-3008

Nixalite of America, PO Box 727, E. Moline, IL 61244; 800/624-1189, 309/755-8771, Fax 800/624-1196

North Bradford Enterprises, RD 1 Box 364, Bradford, ME 04410; 207/327-1237

Novo Nordisk Entotech, 1497 Drew Ave., Davis, CA 95616; 916/757-4700

NPIRS User Services, CERIS, 1231 Cumberland Ave., Ste. A, W. Lafayette, IN 47906-1317; 317/494-6614, Fax 317/4949727

N-VIro, 610 Walnut Ave., Bohemia, NY 11716; 516/567-2628

Oakfleld Apparatus Inc., PO Box 65, Oakfield, WI 53065; 414/583-4114.

Ollver Products Co., 445 Sixth St. NW, Grand Rapids, MI 49504; 616/456-7711

Olson Products Inc., PO Box 1043, Medina, OH 44258; 216/723-3210, Fax 216/7239977

Omnidata Int'I Inc., PO Box 448, Logan, UT 84321; 801/753-7760, Fax 801/753-6756

Organic Control Inc., 5132 Venice Blvd., Los Angeles, CA 90019; 213/937-7444
Organic Plus; 108 Dale Ave. SE, Albuquerque, NM 87105; 800/933-2278, 505/877-

7888, Fax 505/873-9596

Pacific Biocontrol Corp. (Biocontrol Ltd.), 719 Second St., Ste.12, Davis, CA 95616; 800/999-8805, 916/757-2307, Fax 916/7531912

Parker Pest Control Inc., Rte. 2, Box 37, 1 Parker Place, Ponca City, OK 74604; 800/ 654-4541

PBI/Gordon Corp., PO Box 4090, Kansas City, MO 64101; 800/821-7925, 816/421. 4070, Fax 816/474-0462

Peaceful Valley Farm Supply, PO Box 2209, Grass Valley, CA 95945; 916/272-4769

Perma-guard, Inc., PO Box 25282, Albuquerque, NM 87125; 505/883-3061, Fax 505/ 881-6714

Perma Proof Corp, 1927 Howard St., Chicago, IL 60626; 312/764-5559

Pest Management Supply Inc., 311 River Dr., Hadley, MA 01035; 800/272-7672, 413/5497246, Fax 413/549-3930

Pfizer, Inc, 235 East 42nd St,, New York, NY 10017; 212/573-2323

Phero Tech Inc., 7572 Progress Way, Delta, BC CANADA V4G 1E9; 604/940-9944, Fax 604/940-9433

Phillips Fibers Corp., PO Box 66, Greenville, SC 29602; 800/845-5737, 803/242-6600, Fax 803/234-6666

Plant Sciences Inc./Koppert, 342 Green Valley Rd., Watsonville, CA 95076; 408/ 728-7771, Fax 408/728-4967

Plow \& Hearth, 301 Madison Rd., PO Box 830, Orange, VA 22960-0492; 800/627. 1712 , 703/672-1712, Fax 703/672-3612

Poultry Health Services, PO Box 40028, Jacksonville, FL 32203; 904/786-5195

Praxis, 2723 116th Ave., Allegan, MI 49010; 616/673-2793, Fax 616/673-2793

Precision Laboratories Inc., PO Box 127. Northbrook, IL 60065; 800/323-6280, 708/ 498-0800

Premier Enterprises Ltd., 326 Main St., Red Hill, PA 18076; 800/424-2554, Fax 215/679. 4119

Pristine Products, 2311 E. Indian School Rd., Plıoenix, AZ 85016; 800/266-4YOU, 602/ 955-7031

Purity Products, 4 Maxwell Ct., Santa Rosa, CA $95401 ; 707 / 546-2585$

Quantix Systems, 2611 Branch Pike, Cinnaminson, NJ 08077; 800/322-5487, 609/ 786-3080, Fax 609/786-9608

Raven Industries Inc., PO Box 1007, Sioux Falls, SD 57117; 605/336-2750

Reed-Joseph International Co., PO Box 894, 232 Main St., Greenville, MS 38701; 800/ 647-5554, 601/335-5822, Fax 601/335-8850
Reemay Inc., 70 Old Hickory Blvd., PO Box 511, Old Hickory, TN 37138; 800/284-2780, 615/847-7226

REI, PO Box 1938, Sumner, WA 98390; 206/ 395-3780

Rexair Inc., 3221 West Big Beaver, Stc. 200, Troy, MI 48084; 313/643-7222

RF Inter-Sclence Co., PO Box 505, Huntington, NY 11743; 516/421-1342, Fax 516/421-1422

Richmond Fisheries, 8609 Clark Rd., Richmond, IL 60071; 815/675-6545, Fax 815/675-6545

Rincon-VItova Insectaries Inc., PO Box 1555, Ventura, CA 93002; 800/248-BUGS, 805/643-5407 (in CA). Fax 805/643-6267

Ringer Corp., 9959 Valley View Rd., Eden Prairie, MN 55344-3585; 800/423-7544, 612/941-4180, Fax 612/941-5036

Robert Royal, PO Box 108, Midnight, MS 39115; 601/247-4409

Rocky Mountain Insectary, PO Box 152, Pallisade, CO 81526

Rol-Eko Co. Ltd., Horticultural Promotion Bureau, 05-500 Piaseczno, Mysiadto,, Warszawa, POLAND; 022-568241/567386, Fax 022-568241

Roussel UCLAF Corp., 95 Chestnut Ridge Rd., Montvale, NJ 07645; 800/438-5837, Fax 201/307-3281(Fax)

Roxide International Inc., PO Box 249, New Rochelle, NY 10802; 800/431-5500, 914/ 235-5300, Fax 914/235-5328

Safeguard, PO Box 8, New Holland, PA 17557; 800/433-1819

Sandoz Crop Protection, 1300 East Touhy Avenue, Des Plaines, IL 60018; 800/553. 4833, 708/699-1616

San Luis Video Publishing, PO Box 6715, Los Osos, CA 93412; 805/528-8322, Fax 805/528-7227

Santa Cruz Horticultural Supply, PO Box 1534, Moro Bay, CA 93443; 805/772-8262

Sautter \& Stepper, Rosenstr. 19, 72119 Ammerbuch, Altingen, GERMANY; 07032 75501, Fax 07032-74199

Scentry (a division of Ecogen Inc.), 3673 S. Bullard Ave., Ste. 122, Goodyear, AZ 85338 ; 800/548-6531, 602/233-1772; 9323802. Fax 602/932-5614

Seabright Enterprises, 4026 Harlan St., Emeryville, CA 94608; 800/284-7363, 510/ 655-3126, Fax 510/654-7982

Sea Ranch, Route 2 Box 604, Sheridan, AZ 72150

Sespe Creek Insectary, 1400 Grand Ave., Filimore, CA 93015; 805/524-3565

Shelley Enterprises, 18176 Amold Dr., Sonoma, CA 95476; 707/996-3714

SICA-CAF, Fue de Biot, 06560 Valbonne. FRANCE; 93-421789 
Sierra Ag, 2749 E. Malaga, Fresno, CA 93725; 209/233-0585

Sigma Chemical Co., PO Box 14508, St. Louis, MO 63178; 800/325-3010, 314/771. 5750, Fax 800/325-5052

Smith \& Hawken Tools, 117 E. Strawberry Dr., Mill Valley, CA 94941; 800/776-3336, Fax 606/727-1166

Smith Kline Beecham Consumer Brands, PO Box 1467, Pittsburgh, PA 15230; 412/9281000

Sound Merchandise, 7903 E 1st St., PO Box 37. Manchester, WA 98353; 206/565-2362, Fax 206/876-2139

Spalding Laboratories, 760 Printz Rd., Arroyo Grande, CA 93420; 800/845-2847, 805/489-5946

Specialty Products, 977 Waterman Ave., East Providence, RI 02914; 800/556-3484, 401/ 434-1680, Fax 401/431-0237

Spencer-Mead, Inc., 1730 Walton Rd., Ste. 307, Bluebell, PA 19422; 800/645-3696

Steffenson Pest Control, 195 East Sunnyoaks Ave., Campbell, CA 95008; 408/379-5245

Sterling International, Inc., 15916 E. Sprague Ave., Veradale, WA 99037; 800/666-6766, 509/926-6766, Fax 509/928-7313

Sterllng Marlne Products, 18 Label St., Montclair, NJ 07042; 201/783-9800

Stine Seed Co., 2225 Laredo Trail, Adel, IA 50003; 515/677-2605

Streamilght Inc., 1030 W. Germantown Pike, Norristown, PA 19403; 215/631-0600

Strieter Corp, 2100 18th Ave., Ste. 1, Rock Island, IL 61201; 800/747-3337, 309/794. 9800, Fax 309/788-5646

Stromberg's Chlcks \& Game Birds UnllmIted, Box 400, Pine River, MN 56474; 218/ 587-2222

Summit Chemical Co., 7657 Canton Center Dr., Baltimore, MD 21224; 800/227-8664, 410/282-5200, Fax 410/282-7963

Survivalr (a Divlsion of Comasec Inc.), 3001 S. Susan St., Santa Ana, CA 92704; 714/ 545-0410

Sutton Ag Enterprises, 746 Vertin Ave., Salinas, CA 93901; 408/422-9693

Sweetbriar Development, Inc., 1767 San Juan Rd., Watsonville, CA 95076; 408/722-5577 or $726-3256$

TADD Services Corp., 1617 Old County Rd., Ste. 4, Belmont, CA 94002-3940; 800/345TADD, 415/595-5171, Fax 415/595-0121

Tanglefoot Co., 314 Straight Ave. SW, Grand Rapids, MI 49504; 616/459-4139

Target Specialty Products, 1280 North 10 th St., San Jose, CA 95112; 800/767-0719, 408/ 293-6032

Technicide, 11731 Sterling Avc., Ste. H, Riverside, CA 92503; 714/359-5866
Tenax Corp., 4800 E. Monument St., Baltimore, MD 21205; 800/356-8495,410/ 522-7000, Fax 415/522-7015

Termitect II, PO Box 8301, Albuquerque, NM 87198; 505/263-3703; 268-7739

The Aerogen Co. Ltd., Newman Lane, Alton, Hampshire, ENGLAND GU34 2Q; 44-420. 83744, Fax 44-420-80032

The Huge Company, 7625 Page Blvd., Saint Louis, MO 63133; 800/873-4843

The Ladybug Company, 8706 Oro-Quincy Hwy, Berry Creek, CA 95916; 916/5895227

The Naturai Gardening Co., 217 San Anselmo Ave., San Anselmo, CA 94960; 415/456-5060

The Termite Inspector, 25213 Anza Dr., Valencia, CA 91355; 800/322-9661, 805/ 294-2600, Fax 800/622-0261

Thermal Weed Control Systems, Inc., 3403 Hwy. 93, Eau Claire, WI 54701; 715/839. 7242

Tie-Net International, Inc., PO Box 520, West Chicago, IL 60186-0520; 800/7360990, 708/293-3737, Fax 708/293-5303

Tomahawk Live Trap Company, PO Box 323, Tomahawk, WI 54487; 715/453-3550, Fax 715/453-4326

Trécé Inc., 1143 Madison Ln., Salinas, CA 93907-1817; 408/758-0205, Fax 408/7582625

TriCal Blosystems, Inc., PO Box 1327, Hollister, CA 95024; 408/637-0195, Fax 408/637-0273

Turf Seed Inc., PO Box 250, Hubbard, OR 97032; 503/651-2130, Fax 503/651-2351

Unique Insect Control, 5504 Sperry Dr., Citrus Heights, CA 95621; 916/961-7945, Fax 916/967-7082

Unique Products, Inc., 2525 Perimeter Place Dr., Ste. 110, Nashville, TN 37214; 615/ 885-2086

Urban Insect Solutions, 1420 Jondyman Ct., Lexington, KY 40517; 606/273-3747

Urblo s.r.l., via Ghiarino, 640056 Crespellano (BO), ITALY; 051-960244

U.S. Borax, 26877 Tourney Rd., Valencia, CA 91355-1847; 805/287-5400, Fax 800/6264872

USDA Blocontrol of Weeds Laboratory, 800 Buchanan, Albany, CA; 510/559-6082

U.S. Forest Service, USDA, 14th and Independence Aves., Washington, DC 27709

- Valent USA, PO Box 8025, Walnut Creek, CA 94596-8025; 510/256-2700, Fax 510/256-2776

Van Waters \& Rogers, Pest Control

Supplies Dept., 2256 Junction Ave, San Jose, CA $95131 ; 800 / 872-8052$ or $800 / 926$ $8489,408 / 435-8700$

Vet Express Inc., PO Box 155, Hazelhurst, WI
$54531 ; 800 / 458-7656$

Waipuna Systems Ltd., PO Box 106-001, Auckland, New Zealand; 64-9-366 4431, Fax 64-9-366 4470

Waterbury Companies Inc., PO Box 1812 , 32 Mattatuck, Waterbury, CT 06722; 800/ 845-3495, 203/597-1812

Waterford Corp., 404 N. Link Lane, Fort Collins, CO 80522-1513; 800/525-4952, 303/482-0911, Fax 303/482-0934

W. Atlee Burpee Seed Co., 300 Park Ave., Warminster, PA 18974; 215/674-4900

Weed Badger, 5673 95th Ave. SE, Marion, ND 58466; 800/437-3392, 701/778-7511

W.F. Young, Inc., 111 Lyman St., Springfield, MA 01102; 800/628-9653

Whiskers Catfish Farm, 216 Thomton Lane, Bowling Green, KY 42104; 502/842-2555

Whitmire Research Laboratorles, Inc., 3568 Tree Court Industrial Blvd., St. Louis, MO 63122; 800/325-3668, 314/225-5371, Fax 314/225-3739

Wildife Control Technology Inc., $2501 \mathrm{~N}$. Sunnyside Ave. \#103, Fresno, CA 93727; 800/235-0262, 209/294-0262

Wildlife Supply Co., 301 Cass St., Saginaw, MI 48602; 517/799-8100, Fax 517/799-8115

Wilt-Pruf Products Inc., PO Box 469, Essex, CT 06426-0469; 203/767-7033, Fax 203/ 767-7265

Woodstream Corp., 69 N. Locust St., Lititz, PA 17543-0327; 800/800-1819, 717/626. 2125

W. Neudorff, Abt. Nutzorganismen, An der Muhle 3, Postfach 1209, D3254, Emmerthal 1, GERMANY; 49-5155-62460, Fax 495155-6010

Zaklad Produkcjl Enteromotagow "Erna", Nowy Sacz, Wielopole 86, POLAND; 321 78, Fax 0326393

Zeneca Agricultural Products, 1800 Concord Pike, Wilmington, DE 19897; 800/759-4500, 302/886-1000, Fax 302/886-1024

Zoecon Corp., 12200 Denton Dr., Dallas, TX 75234; 800/248-7763

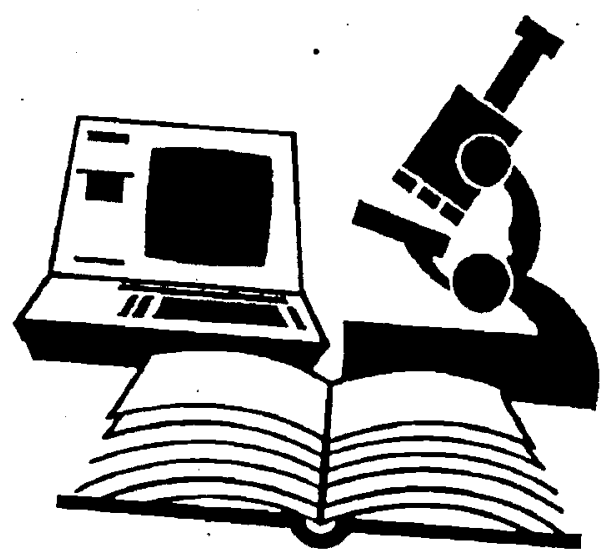




\section{Index}

A

Abamectin 3,13

Aceria malherbae 24

Aeschynomene virgica 24

Agapeta zoegana 24

Agrilus hyperici 24

Agrobacterium radiobacter 19

Alarms \& detonators 21, 22, 23

Amblyseius barkeri 16

Amblyseius californicus 13

Amblyseius cucumeris 13,16

Amblyseius fallacis 13

Amblyseius makenziei 16

Amblyseius spp. 16

Anagnus atomus 12

Anagrus epos 12

Anagrus spp. 12

Anagyrus pseudococei 12

Anaphes spp. 5

Anastatus tenvites 8

Anisopteromalus calandrae 5

Antibiotic, streptomycin 20

Antibiotic, terramycin 20

Antitranspirants 19

Aphelinus abdominalis 4

Aphidius matricariae 4

Aphidius spp. 4

Aphidoletes aphidimyza 4

Aphthona spp. 24

Aphytis lingnanensis 15

Aphytis melinus 15

Apion fuscirostre 24

Apion ulicis 24

Apple scab foreeasting equipment 7 , 19,20

Application equipment 19,24

Artificial diets 18

Attractant for cucumber beetle 5

Attractant for soldier bugs $5,6,7$

B

Bacillus subtilis 20

Bacillus thuringiensis (BT) $5,7,11$

Bacillus thuringiensis israelensis (BTT) 14

Bacillus thuringiensis tenebrionis (BTT) 5

Bait stations 23

Baits 6, 23

Abamectin 8

Borate-based 9

Hydramethylnon 9

Bangastemus orientalis 24

Barriers \& exclusion devices $3,9,12$, $15,21,22,24$

Bat house construction booklet 20

Bat proofing service 20

Bathyplectes curculionis 5

Beckeeping equipment 17

Benzyl benzoate 13

Borate-based insecticides $3,6,7,9$, $11,15,18$

Borate rods 3, 6

Borate termiticide \& wood preservative 6,16

Borate treatments 3

Boric acid 9,15

Bracon hebetor 7
C

Carcinops pumilio 10

Casinaria arjuna 7

Caulk 3, 8, 9

Cephalonomia waterstoni 5

Ceuthorynchidius horridus 24

Ceutorhynchus litura 24

Chilocorus baileyii 15

Chilocorus circumdatus 15

Chilocorus spp. 15

Chrysolina quadrigemina 24

Chrysoperla camea or Chrysopa camea 4

Chrysoperla comanche 4

Chrysoperla rufilabris or Chrysopa rufilabris 4

Chrysoperla spp. 4

Coccophagus lycimnia 15

Coccygomimus disparis 7

Codling moth granulosis vinus 7

Coleophora klimeschiella 24

Coleophora parthenica 24

Combs 9

Comperia merceti 8

Comperiella bifasciata 15

Controlled atmosphere system 5,7

Copper \& sulfur fungicides 20

Cotesia marginiventris 7

Cotesia melanoscela 8

Cotesia plutellae 8

Cryolite 6, 8

Cryptolaemus montrouzieri 13 .

Cunaxoides spp. 15

Cybocephalus sp. 15

D

Dacnusa sibirica 12

Dacnusa spp. 12

Databases 18

Delphastus pusillus 17

Deraeocoris brevis 4,16

Deterrents 21, 22

Alarms \& detonators 21

Light deterrent 21

Reflective tape 21

Roadside highway reflectors 21

Diadegma insularis 8

Diaeretiella rapae 4

Diatomaccous earth $3,4,6,7,8,9,10$, $11,16,17,18$

With pyrethrin $3,4,6,7,8,9,10$, $11,13,16,17$

Diglyphus isaea 12

Diglyphus spp. 12

Disease-resistant roses 19

$\mathbf{E}$

Earthworms 19

Edovum putuleri 5

Electric fencing $21,22,23$

Electrical treatment 6,15

Encarsia formosa 17

Encarsia opulenta 14

Entomopathogenic fungus 14,19

Metarrhizium anisopliae 6,12

Eretmocerus califomicus 17

Euseius rubini 17

Euseius spp. 15, 17

F

Flamers 24

Flashlight with red filters $8,20,22$
Fluon insect barrier 18

Fly bonnet for horses 10

Fumigation bags 9

Furniture covers 13

G

Garlic spray repellent $3,4,11,14,16$, 17

Gcolaclaps spp. 11

Glomus intraradix 19

Glyptapanteles flavicoxis 8

Glyptapanteles indiensis 8

Glyptholaspis confusa 10

Goniozus legneri 8

H

Habitat 4, 19

Head lice products 12

Heat treatment $3,6,15,24$

Herbicidal soap 24

Hippodamia convergens 4

House flies for pollination 19

Hydramethylnon 3,9

Hyles euphorbiac 24

Hypoaspis miles 11,16

I

Information/identification services \& supplies $18,20,23$

Insect growth regulators $4,11,17$

Fenoxycarb 3,6,9

Hydroprene 9

Methoprene 3, 9, 11,14

Insect rearing supplies 19

Insect-parasitic nematodes $5,7,9,10$, 11

Heterorhabditis heliothidis 5, 10 , 11

Heterorhabditis megidis $5,10,11$

Heterorhabditis spp. 5, 10,11

Steinemema bibionis 5,10

Steinernema carpocapsae or Neoaplectana 7

Steinemema carpocapsae or Neoaplectana carpocapsa 5. 10,11

Steinemema spp. 5, 10,11

Insect-resistant turfgrass 6,7

Insecticidal oil $6,8,9,11,13,14,15$, 16,17

Insecticidal soap $3,4,6,7,8,9,11$, $13,14,15,16,17,18$

With citrus oil 13

With pyrethrin 13

Ivermectin 10

With clorsulon 10

L

Landing nets 21

Larinus planus 24

Lemophagus crioceritor 5 .

Leptomastidea abnormis 12

Leptomastix dactylopii 12

Leucoptera spartifoliella 24

Lindorus lophanthae 15

Longitarsus jacobaeae 24

Lydella thompsoni 8

Lysiphlebus testaceipes 4

M

Macrocheles spp. 10
Macrolophus caliginosus 4,17

Mating confusants 3,5

Meigenia mutabilis 5

Mesoseiulus longipes or Phytosciulus longipes 13

Metaphycus helvolus 15

Metarhizium fungal bait station 8

Metaseiulus occidentialis or Galendromus occidenta 13

Meteorus pulchricomis 8

Microbial feeding stimulant 7

Microbial soil amendments 20

Microchelonus blackbumi 8

Microctonus spp. 5

Microlarinus lareynii 24

Microlarinus lypriformus 24

Microplitis plutellae 8

Microscopes 18

Microterys flavus 15

Microwave treatment 6,15

Milky spore disease (Bacillus popilliae) 5

Moisture meters 18

Monitoring/collection equipment 14,

Mosquito fish 14

Mulch 24

Muscidifurax raptor 10

Muscidifurax raptorellus 10

Muscidifurax spp. 10

Muscidifurax zaraptor 10

$\mathbf{N}$

Nasonia vitripennis or Mormoniella vitripennis 10

Neem $4,6,7,8,11,12,13,14,16,17$

Nematicide 14

Nephus reunioni 13

Netting 21, 22

Nosema locustae 11

NPV (nucleopolyhedrosis virus) 7

O

Oberea erythrocephala 24

Odor detector 15

Onthophagus gazella 10

Ooencyrtus kuvanae 8

Ophyra aenescens 10

Orientiseius rickeri or Anthoseius rickeri 13

Orius albidipennis 4, 13,16

Orius insidiosis 13

Orius laevigatus 4, 13,16

Orius majusculus 13,16

Orius spp. 4, 13, 16

Orius tristicolor 4, 13,16

$\mathbf{P}$

Pachycrepoideus vindemiae 10

Pediobius foveolatus 5

Pentalitomastix plethoricus 8

Perillus bioculatus 5

Pesticide detection kits 18, 23

Pheromones \& traps $3,5,6,7,8,10$, $11,14,16,17$

For agricultural \& horticultural insects 5,7

For forestry insects 5,7

For Japanese beetle 5

For stored product insects 5,7

Phlebia gigantea 20 
Phrydiuchus tau 24

Phytoseiulus persimilis 13

Plant disease detection kits 20

Podisus maculiventris 6,8

Poison oakfivy cleanser/prevention 24

Pollinators 19

Praon volucre 4

Propylea quatuordecimpunctata 4

Pseudomonas cepacia 20

Pscudomonas fluorescens 20

Publications \& AV products 18,20 23

Pyemotes tritici 3

P'yrethrin 3, 4, 6, 7, 8, 9, 10, 11, 12 , $14,15,16,17,18$

For head lice 12

For pubic lice 12

Pyrethroid 10, 14, 16, 17

Pyrethrum 4, 8, 10, 17

$\mathbf{R}$

Repellents 10, 14, 17, 21, 22, 23

Repelling balloons 21

Repelling kites 21

Sticky repellent 21

Resmethrin 18

Respirators 19

Rhinocyllus conicus 24

Rigid borescope 15

Row covers 4, 5, 6, 7, 11, 18

Rumina decollata 15

Ryania 5, 6, 7, 8, 9, 11, 16
Sabadilla $5,6,7,8,11$

Screening $3,10,11,12,14,15,16,17$

Scymnus bipunctatus 13

Seed mixture 19

Silica aerogel $3,6,7,8,9,10,14,17$, 18

With ammonium fluosilicate 3,6 , 7,9

With pyrethrin $3,6,8,9,10$

with pyrethrin 7

Skunk deodorant 23

Spalangia cameroni 10

Spalangia corana 10

Spalangia endius 10

Spalangia nigroaenea 10

Sphegigaster sp. 10

Sphenoptera jugoslavica 24

Spurgia esulae 24

Sterile grass carp fish 24

Stethorus punctillum 13

Sticky adhesive $4,10,11,12,15,17$, 21

Sticky barrier 3,7

Streptomyces griseoviridis 20

Streptomycin antibiotic 20

Sympherobius sanctus 13

T

Tannic acid 14

Tenodera aridifolia sinensis 19

Tetanocera spp. 15
Tetrastichus asparagi 5

Tetrastichus hagenowii 8

Tetrastichus julis 5

Thripobius semiluteus 16

Trained dogs $3,6,15$

Traps $4,5,7,8,10,11,12,13,14,15$, $16,17,18,22$

For apple maggot 7,11

For codling moth 7

Glue board trap 3, 8, 22

Light traps $4,5,10,11$

Live traps 22, 23

Solar fly traps 10

Sticky light trap 9

Sticky trap/lape 4, 7,11, 12,14, 16,17

Trap \& attractant 8

Tree wrap 3, 7, 21, 22

Sticky tree band 5

Trichogramma dendrolimi 8

Trichogramma evanescens 8

Trichogramma georgia 8

Trichogramma minutum 8

Trichogramma platneri 8

Trichogramma pretiosum 8

Trichogramma spp. 8

Trichogrammatoidea bactrae 8

Trichosinocalus horridus 24

Trioxys pallidus 4

Trissolcus spp. 6

Turf aerator 20

Tweezers 16
Typhlodromus athiasae 13

Typhlodromus limonicus 13

Tyria jacobacac 24

$\mathbf{U}$

Urolepis rufipes 10

Urophora affinis 24

Urophora cardvi 24

Urophora quadrifásciata 24

Urophora sirunaseva 24

Urophora spp. 24

V

Vacuums $8,9,13,18$

Verticillium lecanii fungus 4,17

Vibratory screens 5

W

Wasp/bee sting medication 18

Weather monitor 19

Weed control fabric 24

Weeder geese 24

Weeding animals 24

Weeding tools 24

Wildlife removal/relocation services 22,23

$\mathbf{X}$

Xylocoris flavipes 6,8

Z

Zeuxidiplosia giardi 24
The Ultimate in Biological Pest Control Guardian Nematodes ${ }^{\mathrm{m}}$ Lawn Patrol $^{\mathrm{m}}$

(Steinemema spp. \& Heteromabditis spp. beneficial nematodes)

Application rate: 1 million per 2,000/3,000 sq.ft. of greenhouse 24 million per acre

Pests: Controls over 250 root zone pests including:

* Cutworms
- European corn borer
- Sod webworms

* Fungus gnats

*orn rootworm

* White grubs

* Thrips

* Strawberry root weevil

Other beneficial Items: Encarsia formosa, Phytosciulus persimilis, Phytoseiulus longipes, Amblyseius californicus, Aphidoletes aphidimyza, Amblyseius mckenziei, Chrysopa carnea (lacewings), Hippodamia convergens (ladybugs), Nosema locustae (Nolo Bait), Mealybug predators, etc. Sticky ribbons, Sticky cards, Sticky stuff, Insect Screens and much more!

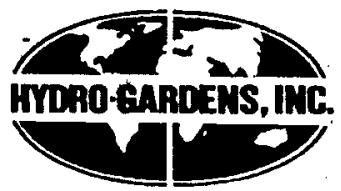

Call TOLL-FREE 1-800-634-6362 for a FREE Catalog

HYDRO-GARDENS, INC.

Your Total Greenhouse Supplier!

P.O. Box 9707, Colorado Springs, CO 80932 * FAX 719-531-0506

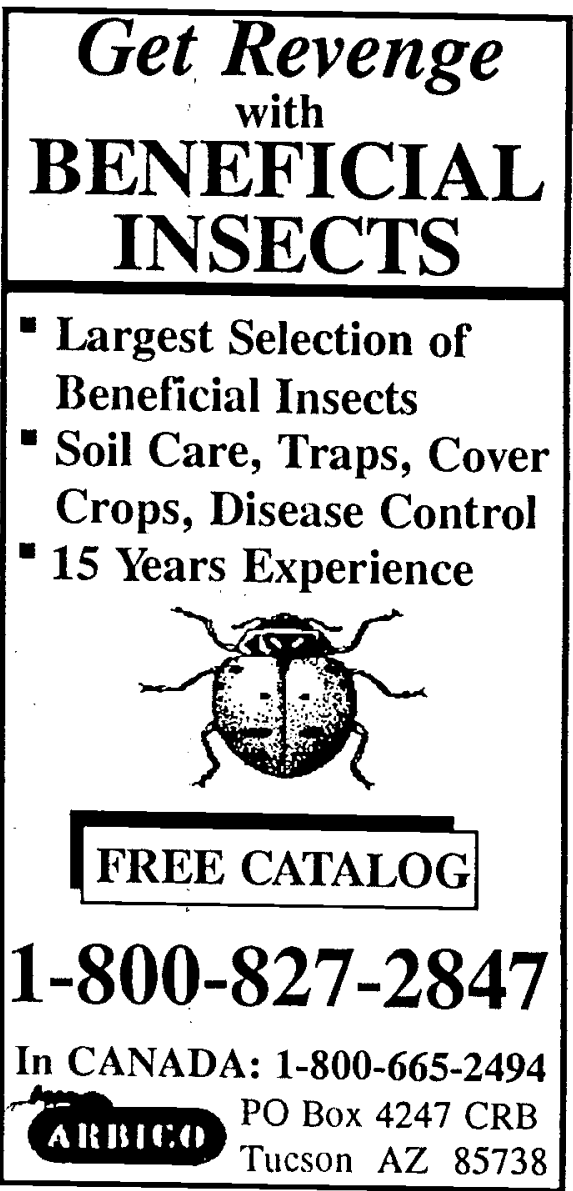




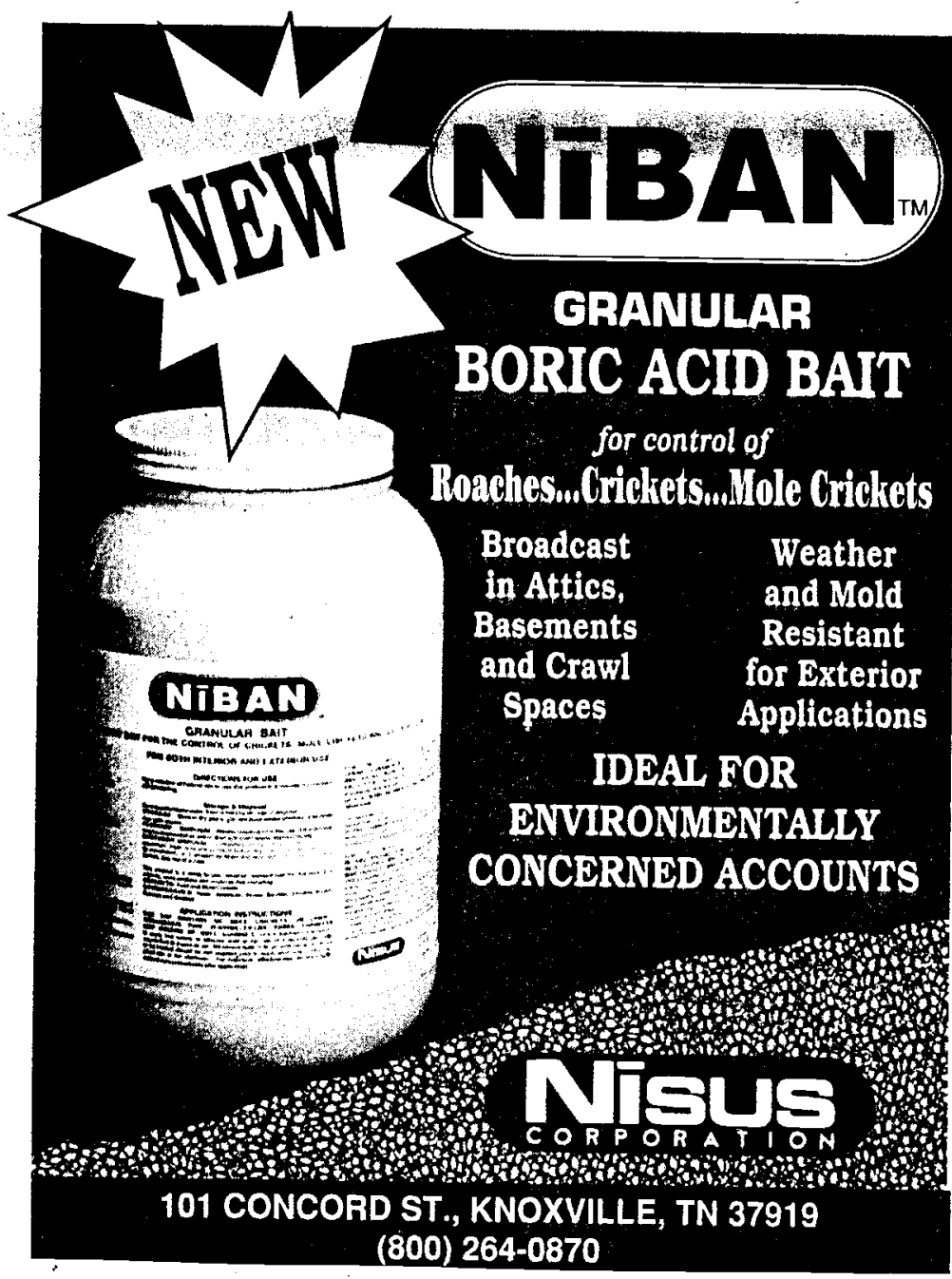

FREE ORGANIC GROWER'S CATALOG

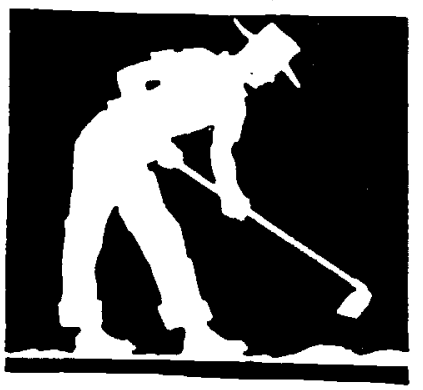

Oldest and largest organic farm and garden supply in the U.S. 120 page catalog, packed with information about organic products E growing techniques. Huge selection of natural pest controls, (including beneficial insects). covercrop, pasture and bulk wildflower seed, natural fertilizers $\varepsilon$ follar feeds, tools, rowcovers, Fall flowerbulbs, seed potatoes, transplant supplies, and more.

\footnotetext{
Peaceful Valley

Farm Supply P.O. Box 2209 P

Grass Valley, CA 95945

(916) 272-4769
}

\section{SNAIL BARR ${ }^{\odot}$}

Non-Toxic Snail \& Slug Control

- Use for flower or vegetable gardens, orchards, nurseries. greenhouses, raised beds \& more.

- Economical, weatherproof, non-toxic and lasts many years.

- No more scarred fruit.

- Over 6 years of successful usage.

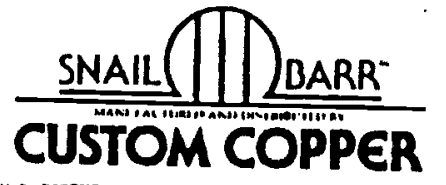

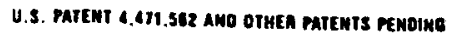

P.O. Box 4939 - Ventura, CA 93004
(805) 647-1652
(619) $749-7132$
FAX
(619) 749-7336

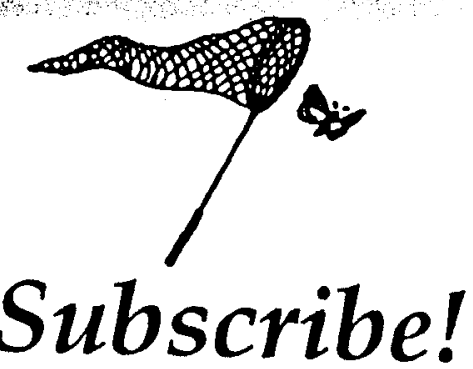

Yes! I want to become a member of the Bio-Integral Resource Center and receive a free subscription to:

\section{The IPM Practitioner}

Enclosed is my check for:

$\square \$ 50 /$ year, Institutions/

Businesses/Libraries

$0 \$ 25 /$ year, Individual

Q \$18/year, Registered Student

* SPECIAL DISCOUNT OFFER

Receive subscriptions to both The IPM Practitioner and the Common Sense Pest Control Quarterly for:

प\$75/year, Institutions/

Businesses/Libraries

$\square \$ 45 /$ year, Individual

Name

Address

City

State Zip

Canadian members, add $\$ 10$ postage; Other foreign, add \$20/air. Foreign orders must be paid in U.S. \$\$ or on an intermational money order.

Enclose your check and mail to:

\section{BIRC}

PO Box 7414

Berkeley, CA 94707

\section{Planning to change your address?}

If so, please notify us six weeks in advance in order not to miss any issues of The IPM Practitioner. Just send a label with a copy of your new address, and we'll do the rest! Thanks: 


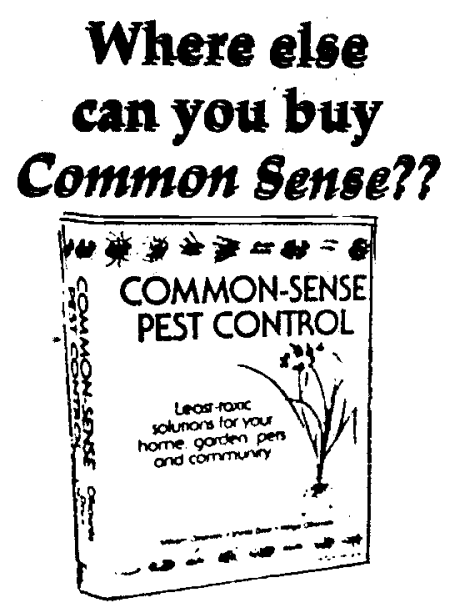

Published by Taunton Press. Hardcover, 715 pages. ISBN \#0-94239-63-2

\section{Common Sense Pest Control}

Least-toxic solutions for your home, garden, pets and community

by William Olkowski, Sheila Daar, and Helga Olkowski

"...the new 'bible' of integrated pest management (IPM)...sure to become a classic handbook for anyone who hates pests but cares about the environment."

- Garden Centers of America

"...a must for born-again bug lovers..."

- The New York Times

"...destined to become the Joy of Cooking of the pest-control world."

$\because$ - Whole Earth Review

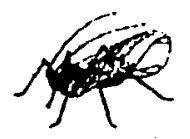

Order it

from BIRC now at

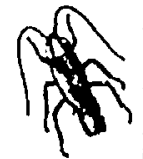

a cost of only $\$ 39.95$

CA residents add $\$ 3.29$ sales tax.

Postage is included - please

allow 4-6 weeks for delivery.

BIRC - PO Box 7414 •

Berkeley, CA • 94707

VISA/Mastercard orders: 510/524-2567 - Fax 510/524-1758

\section{$-$ \\ MreOSTOP:}

blelogieal fUNGIEIBE FBR EBNTRBL OF ROQT BISEASSES:

- a natural product

- effective against Fusaritum, Alternaria and Phomopsis fungi

- on omamentals, vegetables and herbs grown in the greenhouse

- growth stimulating effect

- can be integrated with chemical or biological pesticides

- no special equipment is needed

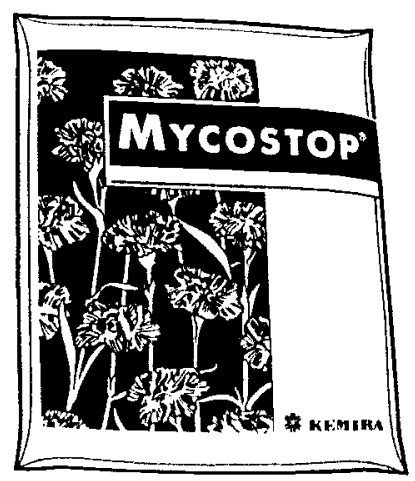

AgBio Development Inc. 9915 Raleigh Street Westminster, CO 80030 Phone: 303-469-9221

Fax: 303-469-9598
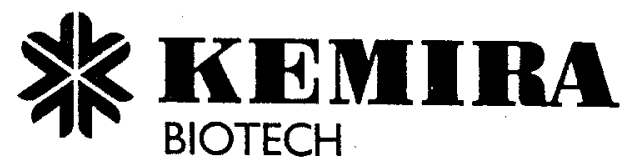

\section{PERMAGUARD $^{\mathrm{TM}}$}

Diatomaceous Earth

- Food Grade for Stored Products -With Pyrethrins for Household
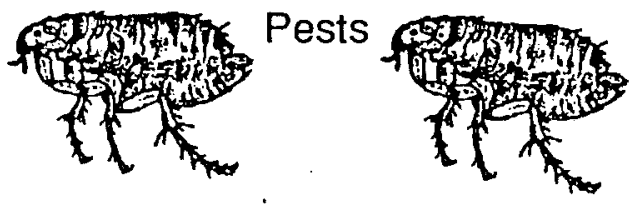

Get Rid of Fleas, Flies, Ticks, Roaches, Grain Weevils and Garden Pests with Nature's Own Organic Pesticide

- Recommended by Sunset Magazine - Used by the Arizona State Fair and Cities of Glendale, Tolleson, and Lake Havasu City -Safe and Easy to Use for Do-it-Yourselfers

PRISTINE PRODUCTS 2311 E. Indian School Rd. Phoenix, AZ 85016 Local: 602/955-7031 Outside Phoenix: 1-800/266-4YOU
PEST CONTROLS MOTHER NATURE WOULD USE NEWS

WHEN THE BUGS MOVE INSIDE

It's that time when pests move in, so keep a close watch out. When they show up, add predators for control, too. Small amounts of predators used now can prevent big problems later on.

Spider-mite Predators $\$ 30 / 1000$ $(5000+\$ 24 / 1000)$ Use 1 for every 20 mites. Whitefly l'arasites $\$ 16.50 / 500$ $(\$ 25 / 1000)$ Use $\cdot 1$ per square foot of greenhouse space. Ladybugs $\$ 6 / 1,500$ $(\$ 20 / 18,000 ; \$ 50 / 72,000)$ Use up to 72,000 /acre.

We ship most orders within hours, and guarantee live delivery. Call or write today for our free brochure "What's Eating My Plants". NATURE'S CONTROL $\triangle \perp \Delta$
P.O. BOX 35

MEDFORD, OR 97501 (503) $899-8318 \quad$ COD'S too FAX (503) 899-9121 


\section{LICE ARE EVERYWHERE ...}

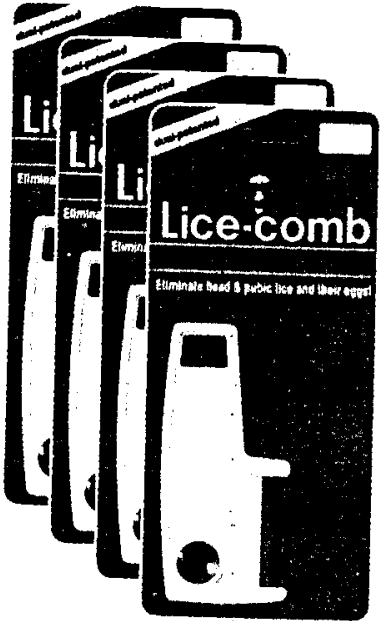

Pediculicides only begin the process of lice treatment. The INNOMED comb makes it work.

Teeth so fine that lice and their eggs are removed without cutting the hair or scratching the scalp.
- Built in 5X magnifier.

- Reusable nickel silver tooth section.

- Dual patented.

\section{The INNOMED LICE COMB ... used WORLDWIDE}

\section{New InnoGel Plus fights crabs} without a scratch.

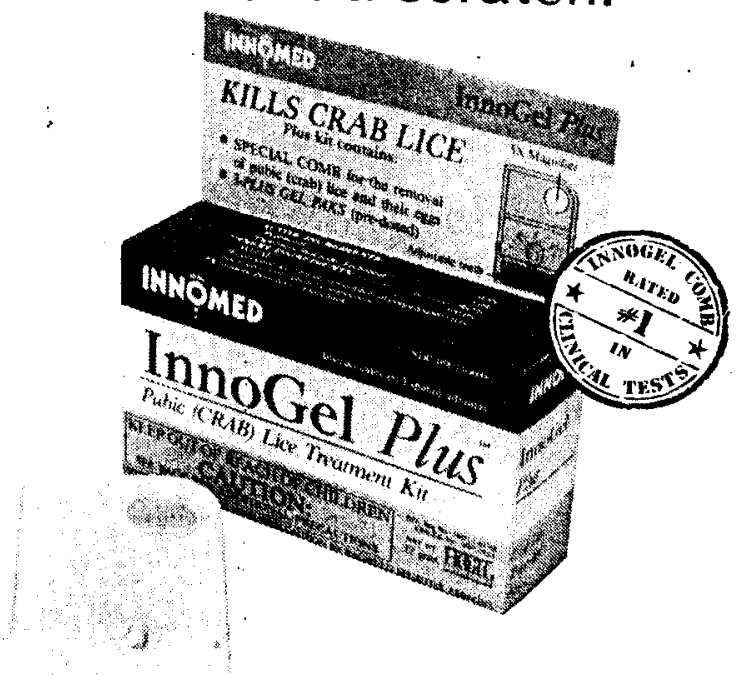

InnoGel Plus is designed exclusively to eliminate crabs.

Getting rid of pubic lice (crabs) is n't as easy as getting them. Crab lice can infest anyone and spread easily with physical contact. The discomfort is legendary. Until now, treatment has been limited to conventional head lice remedies. Finally, there's an adult product for an adult problem.

InnoGel Plus is a complete treatment kit, with three conven- ient gel plus paks and a unique comb for removing crab eggs (nits).

The lrinoGel Plus kit contains everything needed for sale, con-

(c) Hogil Phármaceutical Corp. 1992

The new INNOMED

GROOM 'N FLEA' PET COMB ... effective from every ang/e

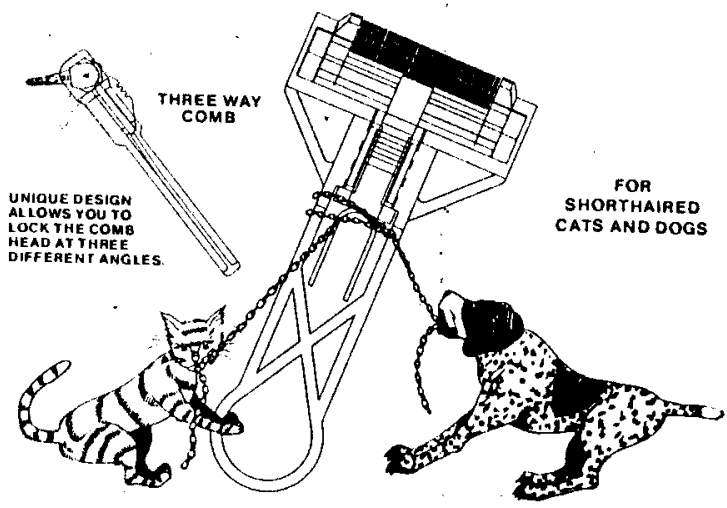

PROFESSIONALLY DESIGNED

PATENTED - The eo pos toon adustable head allows access bes a NEW AGE OESIGN Sale for maximum comlont, Wonl scratch or cul your pet Controls lleas with precision gap controt al 4 microns - Durable laminated nickel sivier leeth

A HEALTHY PET IS A HAPPY PET! 


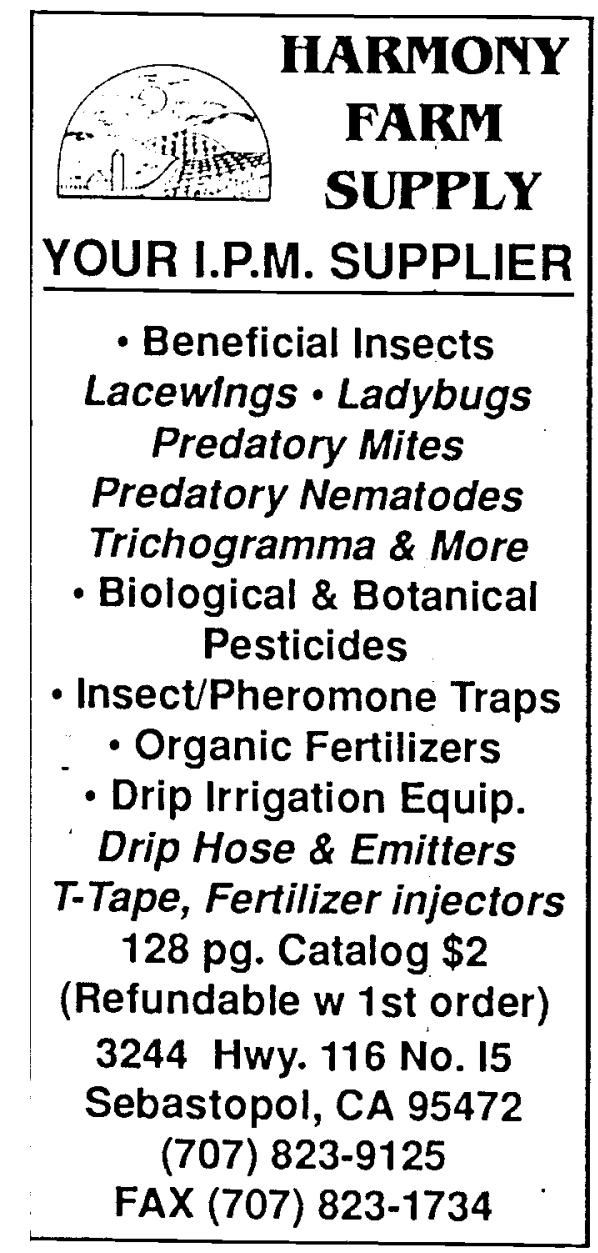

\section{CONTROL PEST BIRDS NOW !!}

Protect your investment! Many product choices and sales outlets for Bird Damage Control.

\section{Write or call} for a free brochure.

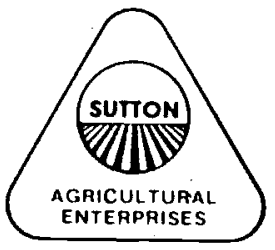

SUTTON AG ENTERPRISES

746 Vertin Avenue Salinas, CA 93901 (408) 422-9693 $\operatorname{FAX}(408) 422-4201$

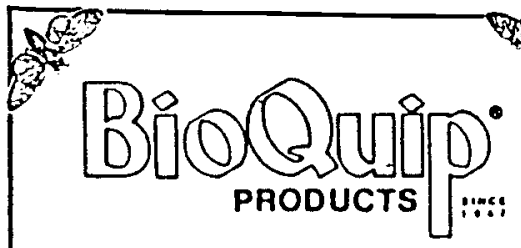

FIRST IN THE FIELD FOR ENTOMOLOGY!

The 1994-1995 BioQuip Catalog with 140 pages is the most extensive ever devoted to the tools and books of Insect Study.

Included are Nets; Cages; Traps; Dissection Tools; Microscopes and Magnifiers; Light Traps; Museum Quality Cabinets and Drawers; Pest I.D. Kits \& Videos; Computer Software; over 900 Books, for Scholars, Amateurs and Children; and much more.

IF YOU ARI SERIOUS ABOUT ENTOMOLOGY, YOU NEED TIIE BIOQUIP CATALOG. NO CIIARGE!

Write, Call or FAX to:

BioQuip Products, Inc. 17803 LaSalle Ave. Gardena, CA, 90248

Phone: (310) 324-0620 Fax: (310) 324-7931

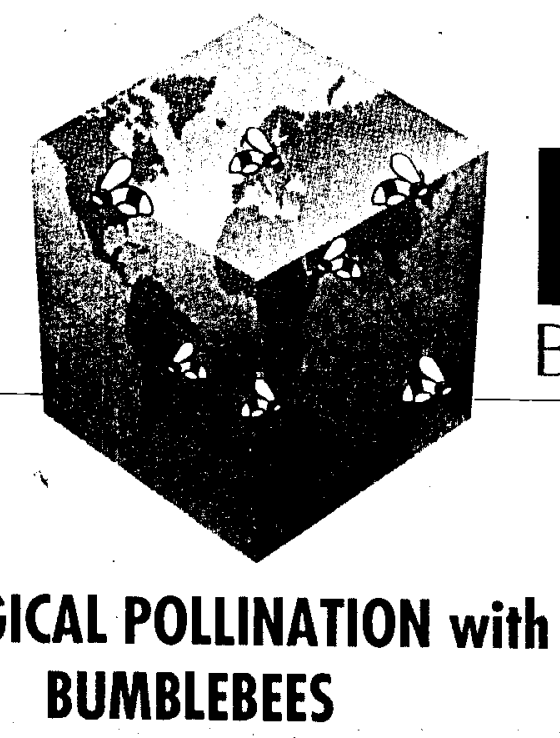

For better fruit set of tomatoes, peppers, courgettes, aubergines, melons, strawberries, blueberries and many other crops

\section{BIOLOGICAL POLLINATION with BUMBLEBEES}

\section{BIOLOGICAL CROP PROTECTION}

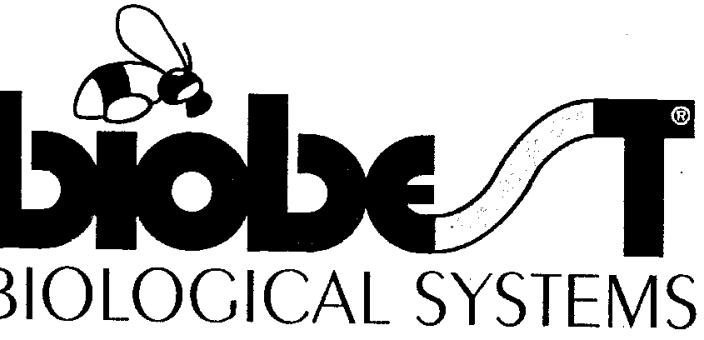
Encarsia, Phytoseiulus, Dacnusa, Diglyphus, Aphidius, Aphidoletes, Aphelinus, Hippodamia, Amblyseius, Orius, Trichogramma, Cryptolaemus, Bacillus thuringiensis, Typhlodromus, insect parasitic nematodes (Heterorhabditis \& Steinernema), blue and yellow glue traps, pheromone traps

For more information, please contact:

Vorldwide:

IIOBEST Biological Systems brba se Velden 18, B-2260 Westerlo, Belgium hone $+32 / 14 / 231701 \cdot \mathrm{Fax}+32 / 14 / 231831$
For the U.S. and Canada:

Beneficial Resources, Inc.

P.O. Box 34, Turbotville PA 17772

Phone (717) 649-6289 • Fax (717) 649-5162 


\section{Perma-Guard, Inc.}

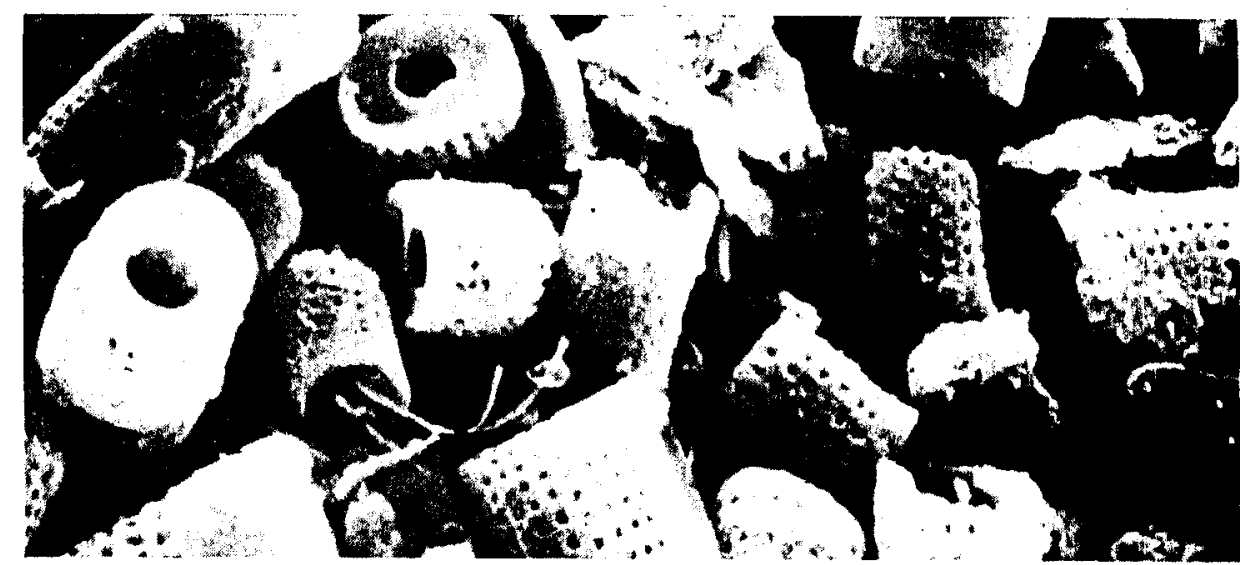

\section{Not All Diatomaceous Earth is the Same!}

Fresh water, salt water, amorphous or crystalline? HMIS rating? Food-grade? Is it high-purity, free of clays, volcanic ash or other foreign materials?

\section{HOW DOES A BUYER KNOW WHAT HE IS GETTING?}

Know your supplier. How long has he been in business? Does he carry liability insurance, does he furnish analysis sheets? Does he have a money-back guarantee? What percentage of crystalline silica is in his product? Is it labelled "Food Grade"?

Wally Tharp, President of Perma-Guard, Inc., started working with diatomaceous earth in 1965 . He has more background in this field than anyone else in the world. His distributors and customers can readily benefit from his knowledge and experience.

Diatomaceous earth can be used as an insecticide:

-Kills bugs by dehydration 9 different ways

-EPA registered for over 100 farm-type bugs

-Insects cannot become immune

-Is highly repellent-keeps bugs away longer

- Mixed with pyrethrum, a time release formula
Diatomaceous earth is an anti-caking agent:

- Keeps particles separated

- Better access to digestive enzymes

- Better metabolism

- Feeds flow better, no clumping

For free information call Wally, Perma-Guard, Inc., at 505/873-3061, or write Perma-Guard, Inc., P.O. Box 25282, Albuquerque, NM 87125, or contact any distributor of Fossil Shell Flour and other Perma-Guard Products:

Agri Energy Resources, Princeton, IL 815/872-1190

Diatoms Plus, Fond du Lac, WI 414/922-4068

Erth-Rite, Gap, PA 717/442-8997

Nature's Best Products, Safford, AL 205/573-9293

Nitron Industries, Fayetteville, AR 800/835-0123

Noble Enterprises, Goodwell, OK 405/349-2569

Perma-Guard, H\&H Distributing, Van, TX 903/963-5784

Pristine Products, Phoenix, AZ 602/955-7031

Hadley Wardlaw, P.O. Box 695 (Hwy 90), Brackettville, TX 78832

Don Weckert, Sioux Falls, SD 605/332-9008 


\section{IN WEECT PACKACING}

Heat-Seal lids, Pouching, Trays, and Sealing Equipment for:

- Rearing - Virus Production

- Bioassay

- Sterile Release

- Crop Resistance Testing
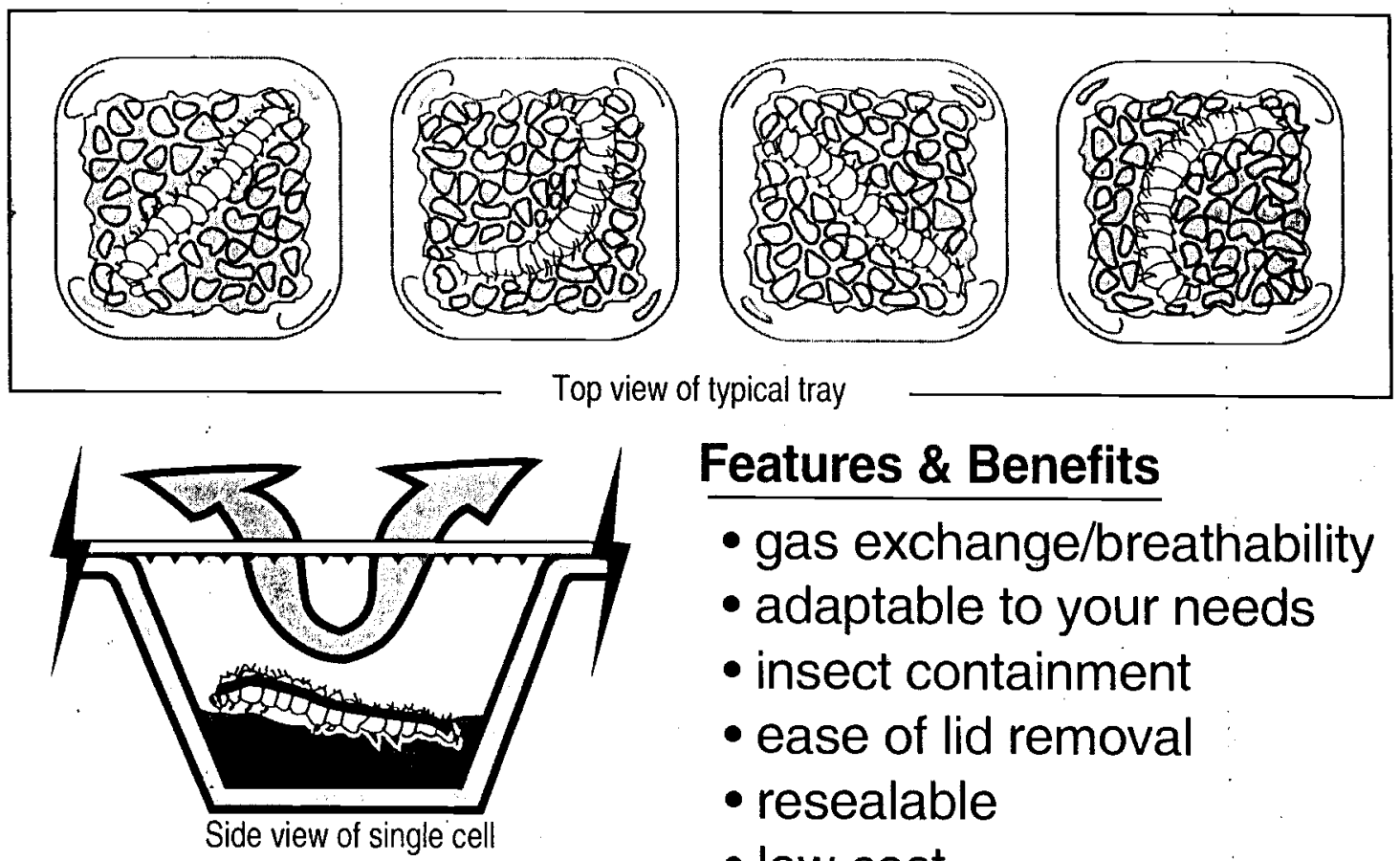

Features \& Benefits

- gas exchange/breathability

- adaptable to your needs

- insect containment

- ease of lid removal

- resealable

- low cost

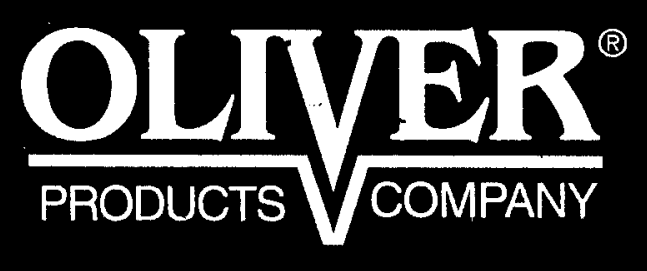
Oliver Products Company
445 Sixth Street, N.W.

Grand Rapids, MI 49504-5298

1-800-253-3893

FAX (616)456-5820 


\section{Classified Ads}

\section{WINTER is RABBIT SEASON!}

Common Sense Pest Control Quarterly, Winter 1994

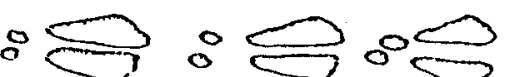

Practical Solutions for Pest Rabbits,

Updates on Termite Control, Mole Control, the 1993 Index

Don't miss out! and More...

Dual Membership \$45/yr BIRC, PO Box 7414, Berkeley, CA 94707

\section{PRODUCTS}

Beneficial Parasitic Nematodes.

Natural environmentally safe way to control insect pests.
Bioshield, PO Box 9068, Fresno, CA 93790 (209) 226-7463 or (209) 297-7480.

\section{READER on LEAST-TOXIC PESTICIDES}

Technical Reviews and Practical Applications for: Silica Gels

Antitranspirants

Boric Acid for Household Pests

Borates for Wood Protection

Diatomaceous Earth

Horticultural Oils

Pyrethrum

Plus...

Fact Sheets on Insecticidal Soaps and Neem

Available for $\$ 12.00$ plus $\$ 2.50$ postage* from:

$$
\begin{gathered}
\text { BIRC, PO Box 7414, Berkeley, CA } 94707 \\
510 / 524-2567 \\
\text { *CA residents add } \$ .99 \text { tax }
\end{gathered}
$$

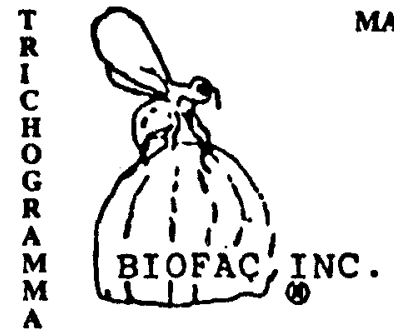

MALCOLM A. MAEDGEN, JR. President

"Natural Pesticides" Por

Stored Products, Fy

Control, Greenhoused

Nursery, Field Crop

Imported Fire Ants

Box 87

Mathis, Texas 78368

ofice - 512/547-3259

bome - 512/547-3169

Blological Solutions for Pest Management

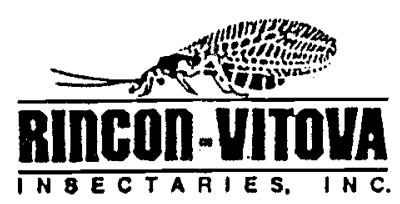

Lacewing (3 species)

Predatory Miles

Thrips Predators

Mealybug Predators

Parasttes of whitefly, aphlds, stink bug, tiles and more.
Codling moth \& avocado strains of Trichogramma platneri Trichogramma minutum Trtchogramma prethosum Trichogrammatoidea bactrae

805-643-5407

800-248-BUGS Outside CA FAX 805-643-6267

PO Box 1555

Ventura, CA 93002

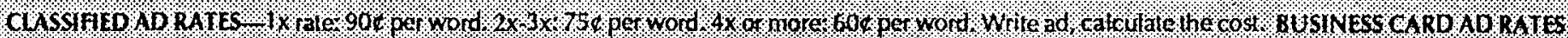

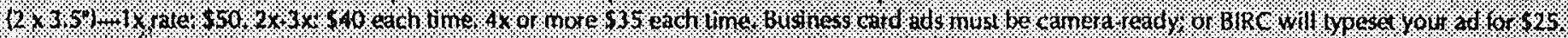

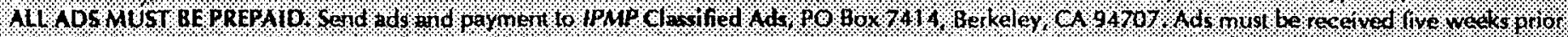

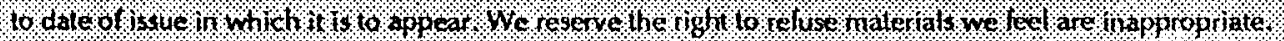

\section{Bio-Integral Resource Center} B $\quad$ I $\quad$ I R $\cdot \mathbf{C}$

P.O. Box 7414, Berkeley, Cailifornia 94707 ADORESS CORRECTION REQUESTED FORWARDING AND RETURN POSTAGE GUARANTEED

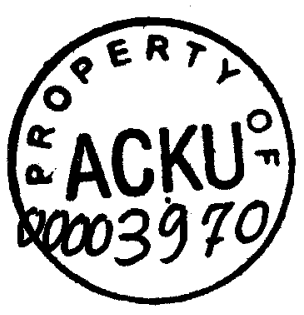

NON-PROFIT ORG. U.S. POSTAGE PAD Berkeley, CA Permit 4442

\section{3}

Mercy Corps International

Thomas M. Brown

3030 SW First Av.

Portland . OR

97201 